\title{
Radical Clock Probes to Determine Carbohydrate Radical Stabilities
}

\author{
Yasemin Mai-Linde and Torsten Linker* \\ Department of Chemistry, University of Potsdam, Karl-Liebknecht-Str. 24-25, 14476 Golm, Germa- \\ ny \\ E-mail: linker@uni-potsdam.de
}

\section{Supporting Information}

1. General information $\quad$ S2

2. General procedure for the synthesis of cyclopropanes 7 and analytical data S2

3. General procedure for the reduction of cyclopropanes 7 to alcohols $8 \quad$ S8

4. Separation of alcohols $x y l o-8$ and $l y x o-8 \quad S 12$

5. General procedure for synthesis of xanthates 9 and analytical data S14

6. General procedure for radical reactions and analytical data $\quad$ S17

$\begin{array}{ll}\text { 7. References } & \text { S23 }\end{array}$

$\begin{array}{lrr}\text { 8. NMR spectra } & \text { S24 }\end{array}$

$\begin{array}{lr}\text { 8a. Cyclopropanes } 7 & \text { S24 }\end{array}$

8b. Alcohols $8 \quad$ S33

8c. Xanthates 9

8d. Products of radical reactions $5 \quad$ S46 


\section{General information}

Melting points were determined by using a Mel-Temp from Electrothermal. TLC was performed using TLC Silica gel 60 F254 aluminium sheets from Merck. Preparative HPLC was performed on a Waters Delta Prep 4000 equipped with an UV/vis absorbance detector. For all compounds a Prontosil 120-10-Si column was used with the dimension 250x20 mm. Solvents with HPLC grade purity were used for all separations. ${ }^{1} \mathrm{H}$ NMR and ${ }^{13} \mathrm{C}$ NMR spectra were measured by using a Bruker Avance $500(500 \mathrm{MHz}, 125 \mathrm{MHz})$ or a Bruker Avance $600(600 \mathrm{MHz}, 150 \mathrm{MHz}) \mathrm{NMR}$ spectrometer. Signals were assigned by two-dimensional methods (COSY, HMQC, HMBC). Configurations were determined by NOESY measurements. IR spectra were recorded as a film by using a Nicolet 6700 FRIR spectrometer from Thermo Electron Corporation. Optical rotations were measured on a JASCO P-1020 digital polarimeter at $589 \mathrm{~nm}$. High-resolution mass spectra were obtained using ESI-Q-TOF techniques. Elemental analysis was performed on a Vario EL III elemental analyzer. Glycals 6 were prepared according to the literature. ${ }^{1}$ All starting materials were used as purchased without further purification.

\section{General procedure for the synthesis of cyclopropanes 7}

Cyclopropanes 7 were synthesized in analogy to literature, ${ }^{2}$ but on a larger scale and by slightly modified procedures and isolation of new side-products. Glycal $6(10 \mathrm{mmol})$ and $\mathrm{Rh}_{2}(\mathrm{OAc})_{4}(88$ $\mathrm{mg}, 0.2 \mathrm{mmol})$ was dissolved in $d r y$ dichloromethane $(100 \mathrm{~mL})$ under an argon atmosphere at room temperature and was shielded from light by aluminium foil. A solution of ethyl diazoacetate ( $3.24 \mathrm{~g}$, $3.62 \mathrm{~mL}, 30 \mathrm{mmol})$ in $d r y$ dichloromethane $(20 \mathrm{~mL})$ was added via syringe pump over a period of 5 h. After TLC showed complete conversion, the solvent was removed at $40{ }^{\circ} \mathrm{C}$. Column chromatography with hexane / methyl-tert.butyl-ether (85:15) afforded the cyclopropanes 7 in analytically pure form. Sometimes mixture fractions had to be separated again by MPLC. The isomers exo-xylo-7 and exo-lyxo-7 had to be separated by HPLC (hexane / ethyl acetate 92:8). An analytical pure fraction of exo-xylo-7 was obtained, exo-lyxo-7 was only isolated as a mixture with exo-xylo-7, but NMR data were determined. The xylo/lyxo isomers could be separated on the stage of the alcohols 8 (see Chapter 3). 

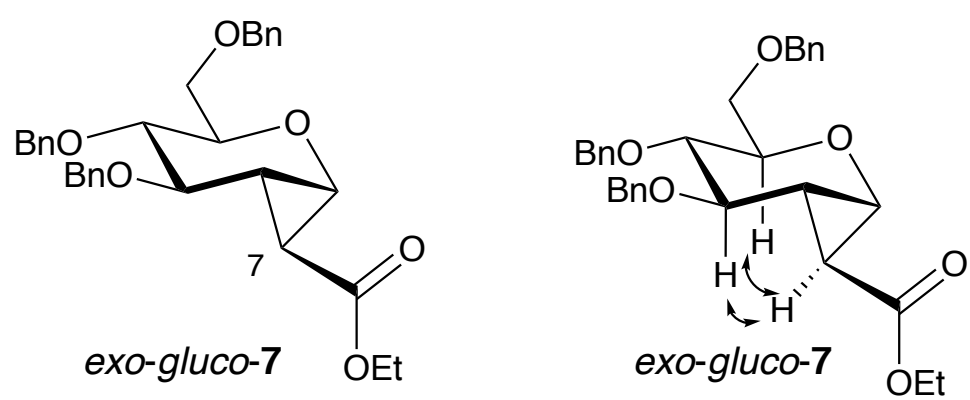

Main product exo-gluco-7: White solid (2.35 g, 47\%). $R_{\mathrm{f}}=0.75$ (hexane/ethyl acetate 8:2); $[\mathrm{a}]_{\mathrm{D}}^{23}+$ $31.6\left(\right.$ c 1.02, $\left.\mathrm{CHCl}_{3}\right)\left(\right.$ Lit. $\left.^{2 \mathrm{a}}[\mathrm{a}]^{20}{ }_{\mathrm{D}}+21.6\right)$; m.p. $54-56{ }^{\circ} \mathrm{C} ;{ }^{1} \mathrm{H} \mathrm{NMR}\left(600 \mathrm{MHz}, \mathrm{CD}_{2} \mathrm{Cl}_{2}\right): \delta 1.14(\mathrm{t}, J$ $\left.=7.1 \mathrm{~Hz}, 3 \mathrm{H}, \mathrm{OCH}_{2} \mathrm{CH}_{3}\right), 1.68(\mathrm{ddd}, J=7.3,5.7,2.1 \mathrm{~Hz}, 1 \mathrm{H}, 2-\mathrm{H}), 1.87(\mathrm{dd}, J=5.7,2.1 \mathrm{~Hz}, 1 \mathrm{H}$, 7-H), 3.45-3.50 (m, 2 H, 4-H, 6-H), 3.61-3.65 (m, 2 H, 5-H, 6'-H), 3.69 (dd, J=6.0, 2.1 Hz, 1 H, 3H), $3.79(\mathrm{dd}, J=7.3,2.1 \mathrm{~Hz}, 1 \mathrm{H}, 1-\mathrm{H}), 3.99\left(\mathrm{dq}, J=10.8,7.1 \mathrm{~Hz}, 1 \mathrm{H}, \mathrm{OCH}_{2} \mathrm{CH}_{3}\right), 4.00(\mathrm{dq}, J=$ 10.8, 7.1 Hz, $\left.1 \mathrm{H}, \mathrm{OC}^{\prime} H_{2} \mathrm{CH}_{3}\right), 4.41\left(\mathrm{~d}, J=12.0 \mathrm{~Hz}, 1 \mathrm{H}, \mathrm{CH}_{2}-\mathrm{Ph}\right), 4.43\left(\mathrm{~d}, J=12.0 \mathrm{~Hz}, 1 \mathrm{H}, \mathrm{CH}_{2^{-}}\right.$ $\mathrm{Ph}), 4.46\left(\mathrm{~d}, J=11.4 \mathrm{~Hz}, 1 \mathrm{H}, \mathrm{CH}_{2}-\mathrm{Ph}\right), 4.49$ (d, $\left.J=11.8 \mathrm{~Hz}, 1 \mathrm{H}, \mathrm{CH}_{2}-\mathrm{Ph}\right), 4.61(\mathrm{~d}, J=11.4 \mathrm{~Hz}, 1$ $\left.\mathrm{H}, \mathrm{CH}_{2}-\mathrm{Ph}\right), 4.63\left(\mathrm{~d}, J=11.8 \mathrm{~Hz}, 1 \mathrm{H}, \mathrm{CH}_{2}-\mathrm{Ph}\right), 7.16-7.21\left(\mathrm{~m}, 15 \mathrm{H}\right.$, arom. H); ${ }^{13} \mathrm{C}$ NMR $(150$ $\mathrm{MHz}, \mathrm{CD}_{2} \mathrm{Cl}_{2}$ ): $\delta 14.4\left(\mathrm{q}, \mathrm{CH}_{2} \mathrm{CH}_{3}\right), 24.8$ (d, C-2), 25.0 (d, C-7), 57.6 (d, C-1), $61.0\left(\mathrm{t}, \mathrm{CH}_{2} \mathrm{CH}_{3}\right)$, 69.8 (t, C-6), 71.7, 73.5, 73.6 (3t, $\left.\mathrm{CH}_{2}-\mathrm{Ph}\right), 75.0$ (d, C-5), 75.8 (d, C-4), 76.5 (d, C-3), 127.8, 127.9, 128.0, 128.1, 128.2, 128.3, 128.6, 128.7, 128.8 (9d, C-arom.), 138.5, 138.7, 138.8 (3s, C-arom.), 171.8 (s, $\mathrm{CO}_{2} \mathrm{Et}$ ); IR (solution) $\tilde{v}=3030,2977,2907,2865,1717,1454,1090,735,697 \mathrm{~cm}^{-1}$; HRMS (ESI-Q-TOF): $\mathrm{m} / z$ calcd for $\mathrm{C}_{31} \mathrm{H}_{35} \mathrm{O}_{6}[\mathrm{M}+\mathrm{H}]^{+}:$503.2434; found 503.2474; Elemental analysis: calcd (\%) for $\mathrm{C}_{31} \mathrm{H}_{34} \mathrm{O}_{6}$ (502.60): C 74.08, H 6.82; found: C 73.70, H 6.62.
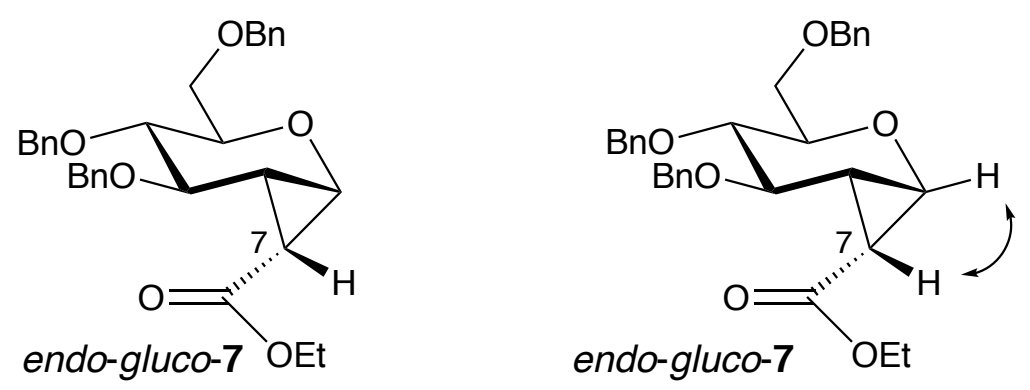

Side product endo-gluco-7: Colorless syrup (900 mg, 18\%). $R_{\mathrm{f}}=0.68$ (hexane/ethyl acetate 8:2); $[\mathrm{a}]^{23}{ }_{\mathrm{D}}+109.7\left(\mathrm{c} 1.01, \mathrm{CHCl}_{3}\right) ;{ }^{1} \mathrm{H} \mathrm{NMR}\left(500 \mathrm{MHz}, \mathrm{CD}_{2} \mathrm{Cl}_{2}\right): \delta 1.15\left(\mathrm{t}, J=7.1 \mathrm{~Hz}, 3 \mathrm{H}, \mathrm{OCH}_{2} \mathrm{CH}_{3}\right)$, 1.34 (ddd, $J=9.2,6.8,4.7 \mathrm{~Hz}, 1 \mathrm{H}, 2-\mathrm{H}), 1.52$ (dd, $J=9.2,6.2 \mathrm{~Hz}, 1 \mathrm{H}, 7-\mathrm{H}), 3.44$ (dd, $J=10.5,2.8$ Hz, 1 H, 6-H), 3.51 (dd, $J=10.5,4.4$ Hz, 1 H, 6'-H), 3.63 (dd, $J=9.4,8.0$ Hz, 1 H, 4-H), 3.76 (ddd, $J=8.0,4.4,2.8 \mathrm{~Hz}, 1 \mathrm{H}, 5-\mathrm{H}), 3.80(\mathrm{dd}, J=6.8,6.2 \mathrm{~Hz}, 1 \mathrm{H}, 1-\mathrm{H}), 4.00(\mathrm{dq}, J=10.8,7.1 \mathrm{~Hz}, 1 \mathrm{H}$, $\mathrm{OCH}_{2} \mathrm{CH}_{3}$ ), 4.01 (dq, $\left.J=10.8,7.1 \mathrm{~Hz}, 1 \mathrm{H}, \mathrm{OC}^{\prime} H_{2} \mathrm{CH}_{3}\right), 4.35$ (d, $\left.J=11.9 \mathrm{~Hz}, 1 \mathrm{H}, \mathrm{CH}_{2}-\mathrm{Ph}\right), 4.40$ $(\mathrm{dd}, J=9.4,4.7 \mathrm{~Hz}, 1 \mathrm{H}, 3-\mathrm{H}), 4.44$ (d, $\left.J=11.9 \mathrm{~Hz}, 1 \mathrm{H}, \mathrm{CH}{ }_{2}-\mathrm{Ph}\right), 4.48$ (d, $J=11.4 \mathrm{~Hz}, 1 \mathrm{H}, \mathrm{CH}_{2^{-}}$ $\mathrm{Ph}), 4.58\left(\mathrm{~d}, J=11.9 \mathrm{~Hz}, 1 \mathrm{H}, \mathrm{CH}_{2}-\mathrm{Ph}\right), 4.69$ (d, $\left.J=11.9 \mathrm{~Hz}, 1 \mathrm{H}, \mathrm{CH}_{2}-\mathrm{Ph}\right), 4.83(\mathrm{~d}, J=11.4 \mathrm{~Hz}, 1$ $\left.\mathrm{H}, \mathrm{CH}_{2}-\mathrm{Ph}\right), 7.18-7.24\left(\mathrm{~m}, 15 \mathrm{H}\right.$, arom. H); ${ }^{13} \mathrm{C} \mathrm{NMR}\left(125 \mathrm{MHz}, \mathrm{CD}_{2} \mathrm{Cl}_{2}\right): \delta 14.5\left(\mathrm{q}, \mathrm{CH}_{2} \mathrm{CH}_{3}\right), 22.7$ 
(d, C-7), 24.6 (d, C-2), 54.0 (d, C-1), 60.7 (t, $\mathrm{CH}_{2} \mathrm{CH}_{3}$ ), 71.1 (t, C-6), 71.8, 73.5, 74.6 (3t, $\mathrm{CH}_{2}-\mathrm{Ph}$ ), 74.5 (d, C-3), 77.3 (d, C-4), 80.0 (d, C-5), 127.8, 127.9, 128.0, 128.1, 128.2, 128.3, 128.4, 128.7, 128.8 (9d, C-arom.), 138.9, 139.2, 139.3 (3s, C-arom.), 169.0 (s, $\mathrm{CO}_{2} \mathrm{Et}$ ); IR (film) $\tilde{v}=3029,2979$, 2903, 2861, 1724, 1453, 1096, 735, $696 \mathrm{~cm}^{-1}$; HRMS (ESI-Q-TOF): $\mathrm{m} / z$ calcd for $\mathrm{C}_{31} \mathrm{H}_{35} \mathrm{O}_{6}$ $[\mathrm{M}+\mathrm{H}]^{+}:$503.2434; found 503.2405; Elemental analysis: calcd (\%) for $\mathrm{C}_{31} \mathrm{H}_{34} \mathrm{O}_{6}(502.60)$ : C 74.08, H 6.82; found: C 73.86, H 6.56 .

\section{manno:}

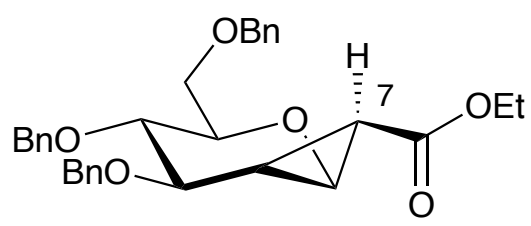

manno-7

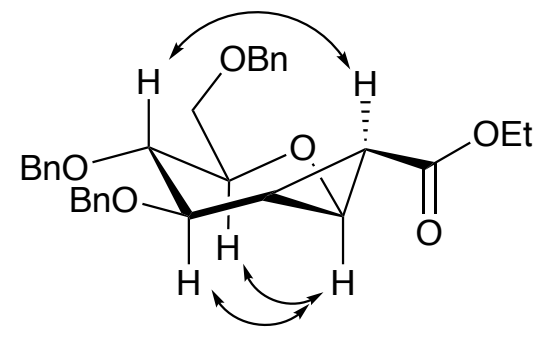

manno-7

Sole isomer manno-7: Colorless syrup (350 mg, 7\%). $R_{\mathrm{f}}=0.32$ (hexane/ethyl acetate 8:2); [a] ${ }^{23}{ }_{\mathrm{D}}^{-}$ 22.0 (c 0.64, $\left.\mathrm{CHCl}_{3}\right) ;{ }^{1} \mathrm{H} \mathrm{NMR}\left(500 \mathrm{MHz}, \mathrm{CD}_{2} \mathrm{Cl}_{2}\right): \delta 1.25$ (t, $\left.J=7.1 \mathrm{~Hz}, 3 \mathrm{H}, \mathrm{OCH}_{2} \mathrm{CH}_{3}\right), 1.95$ (dd, $J=5,9,1,9 \mathrm{~Hz}, 1 \mathrm{H}, 7-\mathrm{H}), 2.13(\mathrm{ddd}, J=7.2,7.0,5.9 \mathrm{~Hz}, 1 \mathrm{H}, 2-\mathrm{H}), 3.36(\mathrm{dd}, J=10.1,7.2 \mathrm{~Hz}, 1 \mathrm{H}$, 4-H), 3.45 (ddd, $J=10.1,4.7,1.7 \mathrm{~Hz}, 1 \mathrm{H}, 5-\mathrm{H}), 3.58$ (dd, $J=10.7,4.7 \mathrm{~Hz}, 1 \mathrm{H}, 6-\mathrm{H}), 3.67$ (dd, $J=$ 10.7, $\left.1.7 \mathrm{~Hz}, 1 \mathrm{H}, 6{ }^{\prime}-\mathrm{H}\right), 4.08$ (dd, $\left.J=7.2,1.9 \mathrm{~Hz}, 1 \mathrm{H}, 1-\mathrm{H}\right), 4.11$ (q, $J=7.1 \mathrm{~Hz}, 2 \mathrm{H}, \mathrm{OCH}_{2} \mathrm{CH}_{3}$ ), $4.17(\mathrm{dd}, J=7.2,7.0 \mathrm{~Hz}, 1 \mathrm{H}, 3-\mathrm{H}), 4.49$ (d, $\left.J=11.9 \mathrm{~Hz}, 1 \mathrm{H}, \mathrm{CH}_{2}-\mathrm{Ph}\right), 4.53$ (d, J=11.9 Hz, $1 \mathrm{H}$, $\left.\mathrm{CH}_{2} \mathrm{Ph}\right), 4.57$ (d, $\left.J=11.1 \mathrm{~Hz}, 1 \mathrm{H}, \mathrm{CH}_{2}-\mathrm{Ph}\right), 4.58$ (d, $\left.J=11.6 \mathrm{~Hz}, 1 \mathrm{H}, \mathrm{CH}_{2}-\mathrm{Ph}\right), 4.72(\mathrm{~d}, J=11.6$ $\left.\mathrm{Hz}, 1 \mathrm{H}, \mathrm{CH}_{2}-\mathrm{Ph}\right), 4.81\left(\mathrm{~d}, J=11.1 \mathrm{~Hz}, 1 \mathrm{H}, \mathrm{CH}_{2}-\mathrm{Ph}\right), 7.22-7.38$ (m, $15 \mathrm{H}$, arom. H); ${ }^{13} \mathrm{C}$ NMR $(125$ MHz, $\mathrm{CD}_{2} \mathrm{Cl}_{2}$ ): $\delta 14.5$ (q, $\mathrm{CH}_{2} \mathrm{CH}_{3}$ ), 26.9 (d, C-2), 27.1 (d, C-7), 61.1 (t, $\mathrm{CH}_{2} \mathrm{CH}_{3}$ ), 62.2 (d, C-1), 69.7 (t, C-6), 71.0, 73.9, 74.8 (3t, $\mathrm{CH}_{2}-\mathrm{Ph}$ ), 78.1 (d, C-3), 78.2 (2d, C-4, C-5), 127.8, 127.9, 128.1, 128.2, 128.3, 128.4, 128.6, 128.7, 128.8 (9d, C-arom.), 138.8, 138.9, 139.1 (3s, C-arom.), 171.4 (s, $\mathrm{CO}_{2} \mathrm{Et}$ ); IR (film) $\tilde{v}=3030,2926,2864,1719,1453,1161,1091,862,737,696 \mathrm{~cm}^{-1}$; HRMS (ESIQ-TOF): $m / z$ calcd for $\mathrm{C}_{31} \mathrm{H}_{35} \mathrm{O}_{6}[\mathrm{M}+\mathrm{H}]^{+}: 503.2434$; found 503.2434; Elemental analysis: calcd (\%) for $\mathrm{C}_{31} \mathrm{H}_{34} \mathrm{O}_{6}$ (502.60): C 74.08, $\mathrm{H}$ 6.82; found: C 73.90, $\mathrm{H} 7.00$.

\section{galacto:}
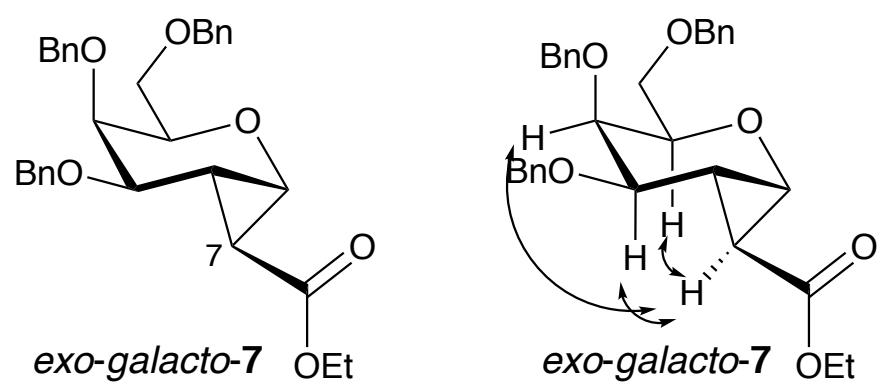
Main product exo-galacto-7: Colorless syrup (2.2 g, 44\%). $R_{\mathrm{f}}=0.34$ (hexane/ethyl acetate 8:2); $[\mathrm{a}]_{\mathrm{D}}^{22}-16.5\left(\mathrm{c} 1.01, \mathrm{CHCl}_{3}\right)\left(\mathrm{Lit}^{2 \mathrm{a}}[\mathrm{a}]^{20}{ }_{\mathrm{D}}-19.2\right) ;{ }^{1} \mathrm{H}$ NMR $\left(600 \mathrm{MHz}, \mathrm{CD}_{2} \mathrm{Cl}_{2}\right): \delta 1.11(\mathrm{t}, J=7.1$ $\left.\mathrm{Hz}, 3 \mathrm{H}, \mathrm{OCH}_{2} \mathrm{CH}_{3}\right), 1.51$ (dd, $\left.J=5.8,2.2 \mathrm{~Hz}, 1 \mathrm{H}, 7-\mathrm{H}\right), 1.78$ (ddd, $J=7.6,5.8,2.6 \mathrm{~Hz}, 1 \mathrm{H}, 2-\mathrm{H}$ ), $3.51(\mathrm{dd}, J=7.3,4.1 \mathrm{~Hz}, 1 \mathrm{H}, 6-\mathrm{H}), 3.53(\mathrm{ddd}, J=5.3,4.1,2.0 \mathrm{~Hz}, 1 \mathrm{H}, 5-\mathrm{H}), 3.56$ (dd, $J=7.3,5.3$ $\mathrm{Hz}, 1 \mathrm{H}, 6$ '-H), 3.59 (dd, $J=2.6,2.2 \mathrm{~Hz}, 1 \mathrm{H}, 3-\mathrm{H}), 3.62$ (dd, $J=2.2,2.0 \mathrm{~Hz}, 1 \mathrm{H}, 4-\mathrm{H}), 3.79$ (dd, $J$ = 7.6, $2.2 \mathrm{~Hz}, 1 \mathrm{H}, 1-\mathrm{H}), 3.97$ (q, $\left.J=7.1 \mathrm{~Hz}, 2 \mathrm{H}, \mathrm{OCH}_{2} \mathrm{CH}_{3}\right), 4.33$ (d, $J=11.9 \mathrm{~Hz}, 1 \mathrm{H}, \mathrm{CH}_{2}-\mathrm{Ph}$ ), $4.38\left(\mathrm{~d}, J=11.9 \mathrm{~Hz}, 1 \mathrm{H}, \mathrm{CH}_{2}-\mathrm{Ph}\right), 4.45$ (d, $\left.J=11.4 \mathrm{~Hz}, 1 \mathrm{H}, \mathrm{CH}_{2}-\mathrm{Ph}\right), 4.53$ (d, $J=11.8 \mathrm{~Hz}, 1 \mathrm{H}$, $\left.\mathrm{CH}_{2}-\mathrm{Ph}\right), 4.65\left(\mathrm{~d}, J=11.8 \mathrm{~Hz}, 1 \mathrm{H}, \mathrm{CH}_{2}-\mathrm{Ph}\right), 4.72$ (d, $\left.J=11.4 \mathrm{~Hz}, 1 \mathrm{H}, \mathrm{CH}_{2}-\mathrm{Ph}\right), 7.14-7.19$ (m, 15 $\mathrm{H}$, arom. H); ${ }^{13} \mathrm{C}$ NMR (150 MHz, $\left.\mathrm{CD}_{2} \mathrm{Cl}_{2}\right): \delta 14.5$ (q, $\left.\mathrm{CH}_{2} \mathrm{CH}_{3}\right), 24.4$ (d, C-2), 25.7 (d, C-7), 57.9 (d, C-1), 61.0 (t, $\mathrm{CH}_{2} \mathrm{CH}_{3}$ ), 69.5 (t, C-6), 71.8, 73.6, 74.5 (3t, $\left.\mathrm{CH}_{2}-\mathrm{Ph}\right), 72.7$ (d, C-3), 74.5 (d, C-5), 75.8 (d, C-4), 127.8, 127.9, 128.0, 128.1, 128.2, 128.3, 128.6, 128.7, 128.8 (9d, C-arom.), 138.6, 138.8, 139.0 (3s, C-arom.), 171.8 (s, $\mathrm{CO}_{2} \mathrm{Et}$ ); IR (film) $\tilde{v}=3030,2980,2907,2868,1717,1454$, 1092, 736, $697 \mathrm{~cm}^{-1}$; HRMS (ESI-Q-TOF): $\mathrm{m} / z$ calcd for $\mathrm{C}_{31} \mathrm{H}_{35} \mathrm{O}_{6}[\mathrm{M}+\mathrm{H}]^{+}:$503.2434; found 503.2425; Elemental analysis: calcd (\%) for $\mathrm{C}_{31} \mathrm{H}_{34} \mathrm{O}_{6}$ (502.60): C 74.08, $\mathrm{H}$ 6.82; found: $\mathrm{C} 73.77, \mathrm{H}$ 7.06 .
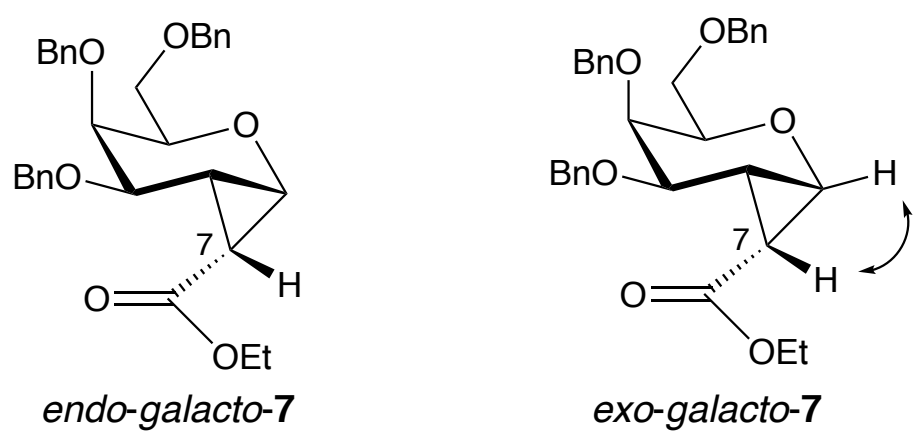

Side product endo-galacto-7: Colorless syrup (500 mg, 10\%). $R_{\mathrm{f}}=0.41$ (hexane/ethyl acetate 8:2); $[\mathrm{a}]^{22}{ }_{\mathrm{D}}+62.7\left(\mathrm{c} 1.04, \mathrm{CHCl}_{3}\right) ;{ }^{1} \mathrm{H}$ NMR $\left(600 \mathrm{MHz}, \mathrm{CD}_{2} \mathrm{Cl}_{2}\right): \delta 1.14\left(\mathrm{t}, J=7.1 \mathrm{~Hz}, 3 \mathrm{H}, \mathrm{OCH}_{2} \mathrm{CH}_{3}\right)$, $1.48(\mathrm{dd}, J=9.5,6.1 \mathrm{~Hz}, 1 \mathrm{H}, 7-\mathrm{H}), 1.55(\mathrm{ddd}, J=9.5,7.4,4.2 \mathrm{~Hz}, 1 \mathrm{H}, 2-\mathrm{H}), 3.46$ (dd, $J=9.4,6.2$ $\mathrm{Hz}, 1 \mathrm{H}, 6-\mathrm{H}), 3.54\left(\mathrm{dd}, J=9.4,7.0 \mathrm{~Hz}, 1 \mathrm{H}, 6{ }^{\prime}-\mathrm{H}\right), 3.80(\mathrm{dd}, J=7.4,6.1 \mathrm{~Hz}, 1 \mathrm{H}, 1-\mathrm{H}), 3.90$ (t, $J=$ $2.2 \mathrm{~Hz}, 1 \mathrm{H}, 4-\mathrm{H}), 3.97$ (ddd, $J=7.0,6.2,2.3 \mathrm{~Hz}, 1 \mathrm{H}, 5-\mathrm{H}), 3.98$ (q, $J=7.1 \mathrm{~Hz}, 2 \mathrm{H}, \mathrm{OCH}_{2} \mathrm{CH}_{3}$ ), $4.22 \mathrm{dd}, J=4.2,2.2 \mathrm{~Hz}, 1 \mathrm{H}, 3-\mathrm{H}), 4.35$ (d, $\left.J=11.8 \mathrm{~Hz}, 1 \mathrm{H}, \mathrm{CH}_{2}-\mathrm{Ph}\right), 4.40$ (d, $J=11.8 \mathrm{~Hz}, 1 \mathrm{H}$, $\left.\mathrm{CH}_{2} \mathrm{Ph}\right), 4.51$ (d, $\left.J=11.3 \mathrm{~Hz}, 1 \mathrm{H}, \mathrm{CH}_{2}-\mathrm{Ph}\right), 4.54$ (d, $\left.J=11.9 \mathrm{~Hz}, 1 \mathrm{H}, \mathrm{CH}_{2}-\mathrm{Ph}\right), 4.63$ (d, $J=11.9$ $\left.\mathrm{Hz}, 1 \mathrm{H}, \mathrm{CH}_{2}-\mathrm{Ph}\right), 4.85$ (d, J=11.3 Hz, $\left.1 \mathrm{H}, \mathrm{CH}_{2}-\mathrm{Ph}\right), 7.16-7.29$ (m, $15 \mathrm{H}$, arom. H); ${ }^{13} \mathrm{C}$ NMR (150 $\mathrm{MHz}, \mathrm{CD}_{2} \mathrm{Cl}_{2}$ ): $\delta 14.5$ (q, $\left.\mathrm{CH}_{2} \mathrm{CH}_{3}\right), 21.4$ (d, C-7), 22.0 (d, C-2), 54.5 (d, C-1), $60.5\left(\mathrm{t}, \mathrm{CH}_{2} \mathrm{CH}_{3}\right.$ ), 70.1 (t, C-6), 71.1, 73.6, 74.7 (3t, $\mathrm{CH}_{2}-\mathrm{Ph}$ ), 72.2 (d, C-3), 73.6 (d, C-4), 78.1 (d, C-5), 127.8, 127.9, 128.0, 128.1, 128.2, 128.3, 128.6, 128.7, 128.8 (9d, C-arom.), 138.8, 138.9, 139.5 (3s, C-arom.), 169.9 (s, $\mathrm{CO}_{2} \mathrm{Et}$ ); IR (film) $\tilde{v}=3029,2980,2927,2866,1721,1454,1375,1094,736,697 \mathrm{~cm}^{-1}$; HRMS (ESI-Q-TOF): $m / z$ calcd for $\mathrm{C}_{31} \mathrm{H}_{35} \mathrm{O}_{6}[\mathrm{M}+\mathrm{H}]^{+}:$503.2434; found 503.2461; Elemental analysis: calcd (\%) for $\mathrm{C}_{31} \mathrm{H}_{34} \mathrm{O}_{6}$ (502.60): C 74.08, $\mathrm{H}$ 6.82; found: $\mathrm{C} 73.89, \mathrm{H}$ 7.01. 


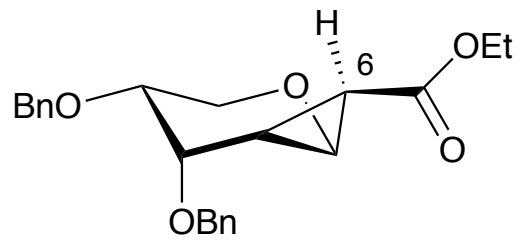

exo-arabino-7

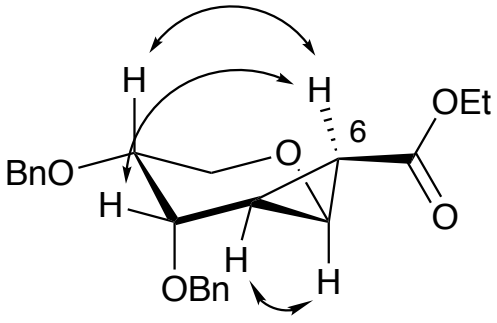

exo-arabino-7

Main product exo-arabino-7: Colorless syrup (1.95 g, 51\%). $R_{\mathrm{f}}=0.48$ (hexane/ethyl acetate 8:2); $[\mathrm{a}]^{24}{ }_{\mathrm{D}}+21.1\left(\mathrm{c} 1.00, \mathrm{CHCl}_{3}\right) ;{ }^{1} \mathrm{H}$ NMR $\left(600 \mathrm{MHz}, \mathrm{CD}_{2} \mathrm{Cl}_{2}\right): \delta 1.23\left(\mathrm{t}, J=7.1 \mathrm{~Hz}, 3 \mathrm{H}, \mathrm{OCH}_{2} \mathrm{CH}_{3}\right)$, 1.57 (dd, $J=6.1,1.9 \mathrm{~Hz}, 1 \mathrm{H}, 6-\mathrm{H}), 2.02$ (ddd, $J=7.1,6.1,2.3 \mathrm{~Hz}, 1 \mathrm{H}, 2-\mathrm{H}), 3.47$ (ddd, $J=8.5$, 7.1, 3.6 Hz, $1 \mathrm{H}, 4-\mathrm{H}), 3.50$ (dd, $\left.J=10.3,3.6 \mathrm{~Hz}, 1 \mathrm{H}, 5_{\text {equ. }}-\mathrm{H}\right), 3.79$ (dd, $J=10.3,8.5 \mathrm{~Hz}, 1 \mathrm{H}, 5_{\text {ax.- }}$ H), $3.89(\mathrm{dd}, J=7.1,1.9 \mathrm{~Hz}, 1 \mathrm{H}, 1-\mathrm{H}), 4.08\left(\mathrm{q}, J=7.1 \mathrm{~Hz}, 2 \mathrm{H}, \mathrm{OCH}_{2} \mathrm{CH}_{3}\right), 4.10$ (dd, $J=7.1,2.3$ $\mathrm{Hz}, 1 \mathrm{H}, 3-\mathrm{H}), 4.58\left(\mathrm{~d}, J=11.9 \mathrm{~Hz}, 1 \mathrm{H}, \mathrm{CH}_{2}-\mathrm{Ph}\right), 4.60\left(\mathrm{~d}, J=11.9 \mathrm{~Hz}, 1 \mathrm{H}, \mathrm{CH}_{2}-\mathrm{Ph}\right), 4.73(\mathrm{~d}, J=$ $\left.11.8 \mathrm{~Hz}, 1 \mathrm{H}, \mathrm{CH}_{2}-\mathrm{Ph}\right), 4.77$ (d, $\left.J=11.8 \mathrm{~Hz}, 1 \mathrm{H}, \mathrm{CH}_{2}-\mathrm{Ph}\right), 7.25-7.40$ (m, $10 \mathrm{H}$, arom. H); ${ }^{13} \mathrm{C} \mathrm{NMR}$ $\left(125 \mathrm{MHz}, \mathrm{CD}_{2} \mathrm{Cl}_{2}\right.$ ): $\delta 14.4$ (q, $\mathrm{CH}_{2} \mathrm{CH}_{3}$ ), 26.0 (d, C-6), 26.9 (d, C-2), 58.7 (d, C-1), 61.0 (t, $\mathrm{CH}_{2} \mathrm{CH}_{3}$ ), 63.4 (t, C-5), 71.8, 72.6 (2t, $\left.\mathrm{CH}_{2}-\mathrm{Ph}\right), 72.0$ (d, C-3), 74.2 (d, C-4), 127.9, 128.0, 128.1, 128.2, 128.6, 128.7 (6d, C-arom.), 138.7, 138.9 (2s, C-arom.), 171.5 (s, $\mathrm{CO}_{2} \mathrm{Et}$ ); IR (film) $\tilde{v}=3030$, 2981, 2936, 2866, 1719, 1454, 1179, 1122, 1057, 739, $700 \mathrm{~cm}^{-1}$; HRMS (ESI-Q-TOF): $\mathrm{m} / z$ calcd for $\mathrm{C}_{23} \mathrm{H}_{27} \mathrm{O}_{5}[\mathrm{M}+\mathrm{H}]^{+}:$383.1858; found 383.1848; Elemental analysis: calcd (\%) for $\mathrm{C}_{23} \mathrm{H}_{26} \mathrm{O}_{5}(382.45)$ : C 72.23, H 6.85; found: C 72.21, H 6.63 .

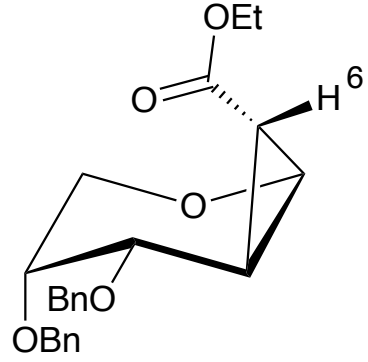

endo-arabino-7

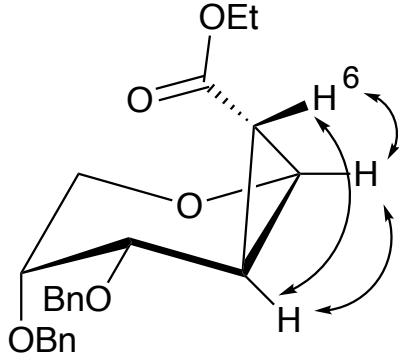

endo-arabino-7

Side product endo-arabino-7: Colorless liquid (300 mg, 8\%). $R_{\mathrm{f}}=0.43$ (hexane/ethyl acetate 8:2); $[\mathrm{a}]_{\mathrm{D}}^{24}-73.3\left(\mathrm{c} 1.03, \mathrm{CHCl}_{3}\right) ;{ }^{1} \mathrm{H}$ NMR $\left(500 \mathrm{MHz}, \mathrm{CD}_{2} \mathrm{Cl}_{2}\right): \delta 1.24\left(\mathrm{t}, J=7.1 \mathrm{~Hz}, 3 \mathrm{H}, \mathrm{OCH}_{2} \mathrm{CH}_{3}\right)$, $1.61(\mathrm{dd}, J=9.5,6.2 \mathrm{~Hz}, 1 \mathrm{H}, 6-\mathrm{H}), 1.64$ (ddd, $J=9.5,7.0,3.8 \mathrm{~Hz}, 1 \mathrm{H}, 2-\mathrm{H}), 3.82$ (dd, $J=10.8,3.1$ Hz, $1 \mathrm{H}, 5-\mathrm{H}), 3.89$ (dd, $J=7.0,6.2 \mathrm{~Hz}, 1 \mathrm{H}, 1-\mathrm{H}), 3.92$ (ddd, $J=3.4,3.1,2.7 \mathrm{~Hz}, 1 \mathrm{H}, 4-\mathrm{H}), 3.94$ (dd, $\left.J=10.8,3.4 \mathrm{~Hz}, 1 \mathrm{H}, 5^{\prime}-\mathrm{H}\right), 4.09$ (q, $\left.J=7.1 \mathrm{~Hz}, 2 \mathrm{H}, \mathrm{OCH}_{2} \mathrm{CH}_{3}\right), 4.37$ (dd, $J=3.8,2.7 \mathrm{~Hz}, 1 \mathrm{H}$, 3-H), $4.64\left(\mathrm{~d}, J=11.8 \mathrm{~Hz}, 1 \mathrm{H}, \mathrm{CH}_{2}-\mathrm{Ph}\right), 4.68$ (d, $\left.J=11.9 \mathrm{~Hz}, 1 \mathrm{H}, \mathrm{CH}-\mathrm{Ph}\right), 4.72(\mathrm{~d}, J=11.9 \mathrm{~Hz}, 1$ $\left.\mathrm{H}, \mathrm{CH}_{2}-\mathrm{Ph}\right), 4.75\left(\mathrm{~d}, J=11.8 \mathrm{~Hz}, 1 \mathrm{H}, \mathrm{Ch} H_{2}-\mathrm{Ph}\right), 7.28-7.34(\mathrm{~m}, 10 \mathrm{H}$, arom. $\mathrm{H}) ;{ }^{13} \mathrm{C}$ NMR $(125$ $\mathrm{MHz}, \mathrm{CD}_{2} \mathrm{Cl}_{2}$ ): $\delta 14.5$ (q, $\left.\mathrm{CH}_{2} \mathrm{CH}_{3}\right), 21.6$ (d, C-6), 21.9 (d, C-2), 54.3 (d, C-1), $60.6\left(\mathrm{t}, \mathrm{CH}_{2} \mathrm{CH}_{3}\right)$, 69.0 (t, C-5), 70.8 (d, C-3), 71.4, 72.3 (2t, $\mathrm{CH}_{2}-\mathrm{Ph}$ ), 73.6 (d, C-4), 127.8, 127.9, 128.0, 128.1, 128.6, 
128.7 (6d, C-arom.), 139.1, 139.4 (2s, C-arom.), 169.6 (s, $\mathrm{CO}_{2} \mathrm{Et}$ ); IR (film) $\tilde{v}=3030,2980,2930$, 2874, 1722, 1454, 1173, 1126, 1096, 1053, 736, $697 \mathrm{~cm}^{-1}$; HRMS (ESI-Q-TOF): $\mathrm{m} / \mathrm{z}$ calcd for $\mathrm{C}_{23} \mathrm{H}_{27} \mathrm{O}_{5}[\mathrm{M}+\mathrm{H}]^{+}:$383.1858; found 383.1830.

xylo:

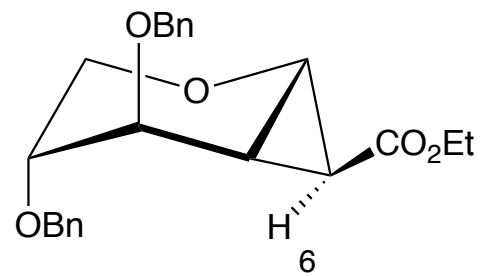

exo-xylo-7

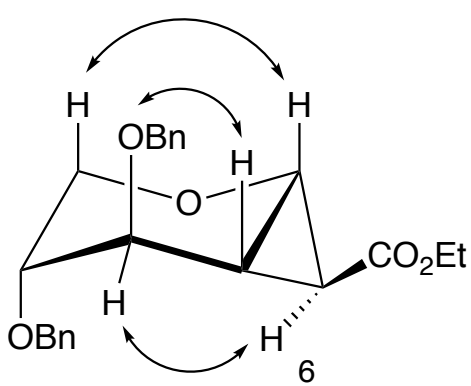

exo-xylo-7

Main product exo-xylo-7: Colorless liquid. $R_{\mathrm{f}}=0.38$ (hexane/ethyl acetate $8: 2$ ); $[\mathrm{a}]^{24} \mathrm{D}-10.5$ (c 0.86, $\left.\mathrm{CHCl}_{3}\right) ;{ }^{1} \mathrm{H}$ NMR $\left(500 \mathrm{MHz}, \mathrm{CD}_{2} \mathrm{Cl}_{2}\right): \delta 1.24\left(\mathrm{t}, J=7.1 \mathrm{~Hz}, 3 \mathrm{H}, \mathrm{OCH}_{2} \mathrm{CH}_{3}\right), 1.86$ (ddt, $J=$ 7.2, 6.0, $1.2 \mathrm{~Hz}, 1 \mathrm{H}, 2-\mathrm{H}), 2.17$ (dd, $J=6.0,1.9 \mathrm{~Hz}, 1 \mathrm{H}, 6-\mathrm{H}), 3.48$ (dddd, $J=3.2,3.0,1.7,1.2 \mathrm{~Hz}$, $1 \mathrm{H}, 4-\mathrm{H}), 3.64$ (dd, $J=12.1,3.2,1.0 \mathrm{~Hz}, 1 \mathrm{H}, 5-\mathrm{H}), 3.70(\mathrm{dd}, J=12.1,1.7 \mathrm{~Hz}, 1 \mathrm{H}, 5 '-\mathrm{H}), 3.92$ (ddd, $J=7.2,1.9,0.7 \mathrm{~Hz}, 1 \mathrm{H}, 1-\mathrm{H}), 3.94$ (dddd, $J=3.0,1.2,1.0,0.7 \mathrm{~Hz}, 1 \mathrm{H}, 3-\mathrm{H}), 4.09$ (q, $J=7.1$ $\left.\mathrm{Hz}, 2 \mathrm{H}, \mathrm{OCH}_{2} \mathrm{CH}_{3}\right), 4.49$ (d, $\left.J=11.8 \mathrm{~Hz}, 1 \mathrm{H}, \mathrm{CH}_{2}-\mathrm{Ph}\right), 4.56$ (d, $\left.J=11.8 \mathrm{~Hz}, 1 \mathrm{H}, \mathrm{CH}_{2}-\mathrm{Ph}\right), 4.57$ (d, $\left.J=11.8 \mathrm{~Hz}, 1 \mathrm{H}, \mathrm{CH}_{2}-\mathrm{Ph}\right), 4.66\left(\mathrm{~d}, J=11.8 \mathrm{~Hz}, 1 \mathrm{H}, \mathrm{CH}_{2}-\mathrm{Ph}\right), 7.25-7.38$ (m, $10 \mathrm{H}$, arom. H); ${ }^{13} \mathrm{C}$ NMR (125 MHz, $\mathrm{CD}_{2} \mathrm{Cl}_{2}$ ): $\delta 14.5$ (q, $\mathrm{CH}_{2} \mathrm{CH}_{3}$ ), 24.5 (d, C-2), 24.7 (d, C-6), 59.0 (d, C-1), 60.9 (t, $\mathrm{CH}_{2} \mathrm{CH}_{3}$ ), 62.7 (t, C-5), 71.1 (d, C-3), 71.4, 72.0 (2t, $\left.\mathrm{CH}_{2}-\mathrm{Ph}\right), 75.2$ (d, C-4), 127.9, 128.0, 128.1, 128.2, 128.7, 128.8 (6d, C-arom.), 138.5, 138.6 (2s, C-arom.), 172.2 (s, $\mathrm{CO}_{2} \mathrm{Et}$ ); IR (film) $\tilde{v}=3665$, 2980, 2902, 1717, 1454, 1295, 1178, 1124, 1079, 1048, 736, $697 \mathrm{~cm}^{-1}$; HRMS (ESI-Q-TOF): $\mathrm{m} / z$ calcd for $\mathrm{C}_{23} \mathrm{H}_{27} \mathrm{O}_{5}[\mathrm{M}+\mathrm{H}]^{+}: 383.1858$; found 383.1843; Elemental analysis: calcd (\%) for $\mathrm{C}_{23} \mathrm{H}_{26} \mathrm{O}_{5}$ (382.45): C 72.23, H 6.85; found: C 72.16, H 7.01.

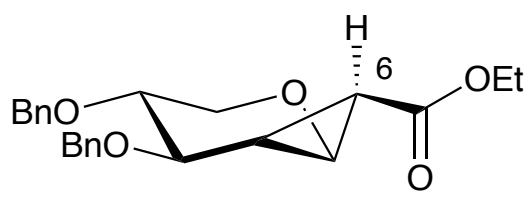

exo-lyxo-7

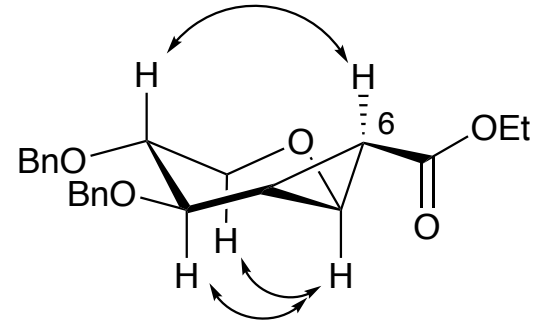

exo-lyxo-7

Side product exo-lyxo-7: Colorless liquid. $R_{\mathrm{f}}=0.38$ (hexane/ethyl acetate 8:2); ${ }^{1} \mathrm{H}$ NMR (500 MHz, $\left.\mathrm{CD}_{2} \mathrm{Cl}_{2}\right): \delta 1.24\left(\mathrm{t}, J=7.2 \mathrm{~Hz}, 3 \mathrm{H}, \mathrm{OCH}_{2} \mathrm{CH}_{3}\right), 1.92(\mathrm{dd}, J=6.0,2.1 \mathrm{~Hz}, 1 \mathrm{H}, 6-\mathrm{H}), 2.07(\mathrm{td}, J=$ 7.3, $6.0 \mathrm{~Hz}, 1 \mathrm{H}, 2-\mathrm{H}), 3.21\left(\mathrm{dd}, J=10.8,10.3 \mathrm{~Hz}, 1 \mathrm{H}, 5_{\mathrm{ax} .}-\mathrm{H}\right), 3.34$ (ddd, $J=10.3,6.9,3.8 \mathrm{~Hz}, 1 \mathrm{H}$, 4-H), 3.61 (dd, $\left.J=10.8,3.8 \mathrm{~Hz}, 1 \mathrm{H}, 5_{\text {equ. }}-\mathrm{H}\right), 4.02$ (ddd, $\left.J=7.3,2.1,1.0 \mathrm{~Hz}, 1 \mathrm{H}, 1-\mathrm{H}\right), 4.07$ (ddd, $J$ $=7.3,6.9,1.0 \mathrm{~Hz}, 1 \mathrm{H}, 3-\mathrm{H}), 4.10\left(\mathrm{q}, J=7.2 \mathrm{~Hz}, 2 \mathrm{H}, \mathrm{OCH}_{2} \mathrm{CH}_{3}\right), 4.61\left(\mathrm{~d}, J=11.7 \mathrm{~Hz}, 1 \mathrm{H}, \mathrm{CH}_{2^{-}}\right.$ 
$\mathrm{Ph}), 4.56\left(\mathrm{~d}, J=11.6 \mathrm{~Hz}, 1 \mathrm{H}, \mathrm{CH}_{2}-\mathrm{Ph}\right), 4.68$ (d, $\left.J=11.7 \mathrm{~Hz}, 1 \mathrm{H}, \mathrm{CH} H_{2}-\mathrm{Ph}\right), 4.71(\mathrm{~d}, J=11.6 \mathrm{~Hz}, 1$ $\mathrm{H}, \mathrm{CH}_{2}-\mathrm{Ph}$ ), 7.26-7.34 (m, $10 \mathrm{H}$, arom. H); ${ }^{13} \mathrm{C}$ NMR (125 MHz, $\mathrm{CD}_{2} \mathrm{Cl}_{2}$ ): $\delta 14.5$ (q, $\left.\mathrm{CH}_{2} \mathrm{CH}_{3}\right), 26.3$ (d, C-6), 26.4 (d, C-2), 61.1 (t, $\mathrm{CH}_{2} \mathrm{CH}_{3}$ ), 62.6 (d, C-1), 66.3 (t, C-5), 71.3, 73.0 (2t, $\left.\mathrm{CH}_{2}-\mathrm{Ph}\right), 76.0$ (d, C-3), 77.2 (d, C-4), 128.0, 128.1, 128.4, 128.7, 128.8, 128.9 (6d, C-arom.), 139.0, 139.1 (2s, Carom.), 171.5 (s, $\mathrm{CO}_{2} \mathrm{Et}$ ); IR (film) $\tilde{v}=3030,2976,2920,2865,1718,1454,1427,1306,1257$, 1177, 1122, 1091, 996, 736, $697 \mathrm{~cm}^{-1}$; HRMS (ESI-Q-TOF): $\mathrm{m} / z$ calcd for $\mathrm{C}_{23} \mathrm{H}_{27} \mathrm{O}_{5}[\mathrm{M}+\mathrm{H}]^{+}$: 383.1858; found 383.1847.

\section{General procedure for the reduction of cyclopropanes 7 to alcohols 8}

Alcohols 8 were synthesized in analogy to the literature for the gluco-8 isomer. $^{3}$ A suspension of $\mathrm{LiAlH}_{4}(209 \mathrm{mg}, 5.5 \mathrm{mmol})$ in $d r y$ diethyl ether $(80 \mathrm{~mL})$ was cooled to $0{ }^{\circ} \mathrm{C}$. A solution of cyclopropane $7(5 \mathrm{mmol})$ in dry diethyl ether $(60 \mathrm{~mL})$ was added slowly at this temperature and the mixture was stirred for $1 \mathrm{~h}$. The reaction was quenched with ice $(30 \mathrm{~mL})$ and filtered through a celite pad. The celite pad was washed with diethyl ether $(3 \times 40 \mathrm{~mL})$, the aqueous phase was removed, the combined organic layers were dried with $\mathrm{Na}_{2} \mathrm{SO}_{4}$, and the solvent was removed at $40{ }^{\circ} \mathrm{C}$. Column chromatography with hexane / methyl-tert.butyl-ether (40:60) afforded the alcohols 8 in analytically pure form. The alcohols $x y l o-8$ and lyxo-8 were synthesized by reduction of a mixture of esters exoxylo-7 and exo-lyxo-7 and isolated in analytically pure form on a small scale by separation by HPLC (hexane / isopropanol 98:2). A larger amount of pure xylo-8 was obtained by transformation of an isomeric alcohol mixture into 3,5-dinitrobenzoates, separation by column chromatography, and subsequent saponification (see Chapter 4 for details).

gluco-8:
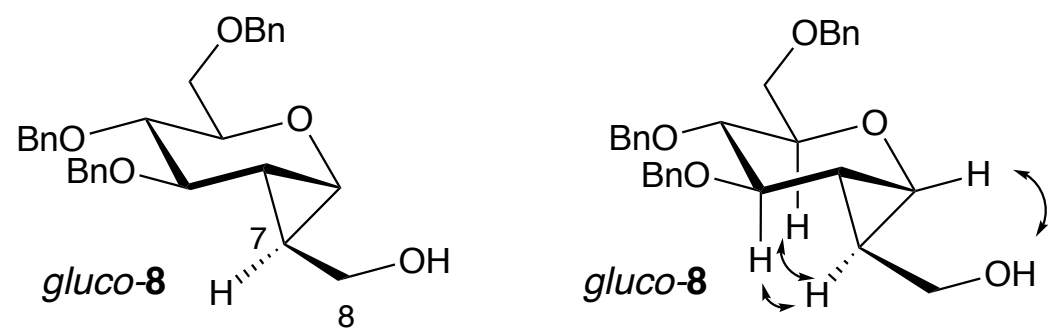

White solid $(2.0 \mathrm{~g}, 98 \%) . R_{\mathrm{f}}=0.19$ (hexane/ethyl acetate $\left.1: 1\right) ;[\mathrm{a}]^{23}{ }_{\mathrm{D}}+37.3$ (c 1.04, $\left.\mathrm{CHCl}_{3}\right)\left(\right.$ Lit. $^{3}$ $\left.[\mathrm{a}]^{20}{ }_{\mathrm{D}}+36\right)$; m.p. $64-65{ }^{\circ} \mathrm{C} ;{ }^{1} \mathrm{H}$ NMR $\left(600 \mathrm{MHz}, \mathrm{CD}_{2} \mathrm{Cl}_{2}\right): \delta 0.94(\mathrm{ddd}, J=7.2,5.5,3.5 \mathrm{~Hz}, 1 \mathrm{H}, 2-$ H), 1.35 (dddd, $J=7.4,6.6,5.5,2.3 \mathrm{~Hz}, 1 \mathrm{H}, 7-\mathrm{H}), 1.40$ (bs, $1 \mathrm{H}, \mathrm{OH}), 3.36$ (dd, $J=11.5,7.4 \mathrm{~Hz}, 1$ H, 8-H), 3.42 (dd, $J=7.2,2.3 \mathrm{~Hz}, 1 \mathrm{H}, 1-\mathrm{H}), 3.44$ (dd, $J=11.5,6.6 \mathrm{~Hz}, 1 \mathrm{H}, 8$ '-H), 3.50 (dd, $J=7.1$, 
$5.7 \mathrm{~Hz}, 1 \mathrm{H}, 4-\mathrm{H}), 3.54$ (dd, $J=10.2,3.5 \mathrm{~Hz}, 1 \mathrm{H}, 6-\mathrm{H}), 3.65$ (dd, $J=7.1,3.5 \mathrm{~Hz}, 1 \mathrm{H}, 3-\mathrm{H}), 3.70$ (dd, $J=10.2,6.5 \mathrm{~Hz}, 1 \mathrm{H}, 6$ '-H), 3.75 (ddd, $J=6.5,5.7,3.5 \mathrm{~Hz}, 1 \mathrm{H}, 5-\mathrm{H}), 4.50$ (d, $J=11.9 \mathrm{~Hz}, 1 \mathrm{H}$, $\left.\mathrm{CH}_{2}-\mathrm{Ph}\right), 4.53\left(\mathrm{~d}, J=11.9 \mathrm{~Hz}, 1 \mathrm{H}, \mathrm{CH}_{2}-\mathrm{Ph}\right), 4.56\left(\mathrm{~d}, J=11.5 \mathrm{~Hz}, 1 \mathrm{H}, \mathrm{CH}_{2}-\mathrm{Ph}\right), 4.63(\mathrm{~d}, J=11.8$ $\left.\mathrm{Hz}, 1 \mathrm{H}, \mathrm{CH}_{2}-\mathrm{Ph}\right), 4.75\left(\mathrm{~d}, J=11.5 \mathrm{~Hz}, 1 \mathrm{H}, \mathrm{CH}_{2}-\mathrm{Ph}\right), 4.75\left(\mathrm{~d}, J=11.8 \mathrm{~Hz}, 1 \mathrm{H}, \mathrm{CH}_{2}-\mathrm{Ph}\right), 7.26-7.39$ (m, $15 \mathrm{H}$, arom. H); ${ }^{13} \mathrm{C}$ NMR (150 MHz, $\mathrm{CD}_{2} \mathrm{Cl}_{2}$ ): $\delta 20.0$ (d, C-2), 27.0 (d, C-7), $53.9(\mathrm{~d}, \mathrm{C}-1), 63.2$ (t, C-8), 70.4 (t, C-6), 71.5, 73.5, 73.6 (3t, $\mathrm{CH}_{2}-\mathrm{Ph}$ ), 77.0 (d, C-5), 77.1 (d, C-4), 79.0 (d, C-3), 127.8, 127.9, 128.0, 128.1, 128.2, 128.6, 128.7, 128.7, 128.8 (9d, C-arom.), 138.8, 138.9, 139.0 (3s, Carom.); IR (solution) $\tilde{v}=3437(\mathrm{OH}), 3062,3030,2865,1454,1367,1094,1028,738,699 \mathrm{~cm}^{-1}$; HRMS (ESI-Q-TOF): $\mathrm{m} / z$ calcd for $\mathrm{C}_{29} \mathrm{H}_{33} \mathrm{O}_{5}[\mathrm{M}+\mathrm{H}]^{+}: 461.2328$; found 461.2223; Elemental analysis: calcd (\%) for $\mathrm{C}_{29} \mathrm{H}_{32} \mathrm{O}_{5}$ (460.57): C 75.63, H 7.00; found: C 75.27, H 7.15.

\section{manno 8:}

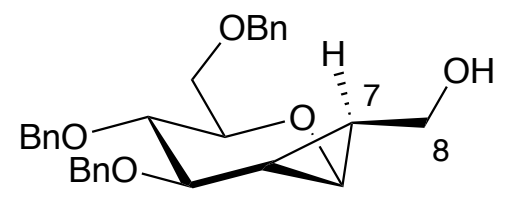

manno-8

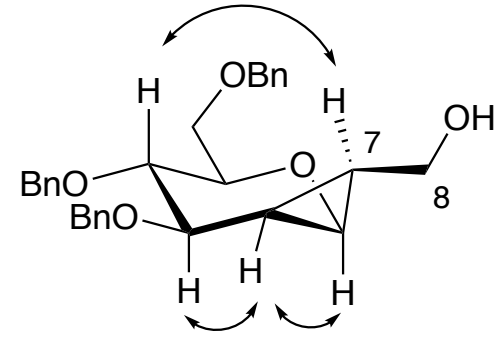

manno-8

White solid (2.0 g, 98\%). $R_{\mathrm{f}}=0.17$ (hexane/ethyl acetate 1:1); $[\mathrm{a}]^{23}{ }_{\mathrm{D}}-53.6$ (c 1.00, $\left.\mathrm{CHCl}_{3}\right) ;$ m.p. 56 ${ }^{\circ} \mathrm{C} ;{ }^{1} \mathrm{H}$ NMR $\left(600 \mathrm{MHz}, \mathrm{CD}_{2} \mathrm{Cl}_{2}\right): \delta 1.41(\mathrm{td}, J=6.9,6.4 \mathrm{~Hz}, 1 \mathrm{H}, 2-\mathrm{H}), 1.44(\mathrm{tdd}, J=6.4,4.2,2.5$ Hz, $1 \mathrm{H}, 7-\mathrm{H}), 1.89$ (bs, $1 \mathrm{H}, \mathrm{OH}), 3.36$ (dd, $J=10.0,6.9 \mathrm{~Hz}, 1 \mathrm{H}, 4-\mathrm{H}), 3.40-3.46$ (m, 3 H, 5-H, 8H), $3.59(\mathrm{dd}, J=10.6,5.1 \mathrm{~Hz}, 1 \mathrm{H}, 6-\mathrm{H}), 3.71(\mathrm{dd}, J=6.9,2.5 \mathrm{~Hz}, 1 \mathrm{H}, 1-\mathrm{H}), 3.72$ (dd, $J=10.6,1.9$ Hz, $1 \mathrm{H}, 6$ '-H), 4.18 (t, $J=6.9 \mathrm{~Hz}, 1 \mathrm{H}, 3-\mathrm{H}), 4.52$ (d, $J=11.8 \mathrm{~Hz}, 1 \mathrm{H}, \mathrm{CH}-\mathrm{Ph}), 4.56$ (d, $J=11.8$ $\left.\mathrm{Hz}, 1 \mathrm{H}, \mathrm{CH}_{2}-\mathrm{Ph}\right), 4.58\left(\mathrm{~d}, J=11.1 \mathrm{~Hz}, 1 \mathrm{H}, \mathrm{CH}_{2}-\mathrm{Ph}\right), 4.61\left(\mathrm{~d}, J=11.5 \mathrm{~Hz}, 1 \mathrm{H}, \mathrm{CH}_{2}-\mathrm{Ph}\right), 4.84(\mathrm{~d}, J$ $\left.=11.1 \mathrm{~Hz}, 1 \mathrm{H}, \mathrm{CH}_{2} \mathrm{Ph}\right), 4.84\left(\mathrm{~d}, J=11.5 \mathrm{~Hz}, 1 \mathrm{H}, \mathrm{CH}_{2}-\mathrm{Ph}\right), 7.26-7.32(\mathrm{~m}, 15 \mathrm{H}$, arom. $\mathrm{H}) ;{ }^{13} \mathrm{C}$ NMR (150 MHz, CD $\mathrm{Cl}_{2}$ ): $\delta 21.1$ (d, C-2), 27.7 (d, C-7), 59.4 (d, C-1), 63.3 (t, C-8), 69.9 (t, C-6), 70.3, 73.7, 74.4 (3t, $\mathrm{CH}_{2}-\mathrm{Ph}$ ), 77.7 (d, C-5), 78.7 (d, C-4), 78.9 (d, C-3), 127.8, 127.9, 128.0, 128.1, 128.2, 128.3, 128.4, 128.6, 128.7 (9d, C-arom.), 138.8, 139.1, 139.2 (3s, C-arom.); IR (solution) $\tilde{v}=$ 3455 (OH), 3031, 2982, 2907, 2868, 1496, 1453, 1075, 1026, 734, $697 \mathrm{~cm}^{-1}$; HRMS (ESI-Q-TOF): $m / z$ calcd for $\mathrm{C}_{29} \mathrm{H}_{33} \mathrm{O}_{5}[\mathrm{M}+\mathrm{H}]^{+}: 461.2328$; found 461.2313; Elemental analysis: calcd (\%) for $\mathrm{C}_{29} \mathrm{H}_{32} \mathrm{O}_{5}$ (460.57): C 75.63, H 7.00; found: C 75.29, H 7.22. 

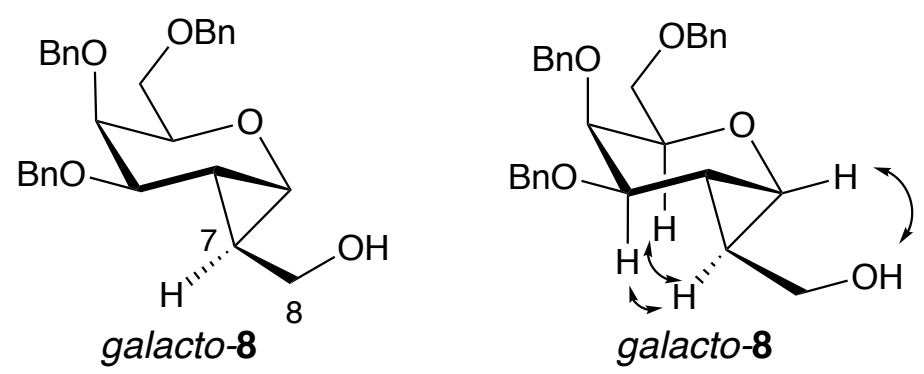

Colorless syrup $(2.0 \mathrm{~g}, 98 \%) . R_{\mathrm{f}}=0.26$ (hexane/ethyl acetate $\left.1: 1\right) ;[\mathrm{a}]^{24}{ }_{\mathrm{D}}+12.2\left(\mathrm{c} 1.03, \mathrm{CHCl}_{3}\right) ;{ }^{1} \mathrm{H}$ NMR (600 MHz, $\mathrm{CD}_{2} \mathrm{Cl}_{2}$ ): $\delta 1.07$ (dddd, $\left.J=7.4,6.8,5.9,2.5 \mathrm{~Hz}, 1 \mathrm{H}, 7-\mathrm{H}\right), 1.13$ (ddd, $J=7.5,5.9$, $3.1 \mathrm{~Hz}, 1 \mathrm{H}, 2-\mathrm{H}), 1.60$ (bs, $1 \mathrm{H}, \mathrm{OH}), 3.39$ (dd, $J=11.5,7.4 \mathrm{~Hz}, 1 \mathrm{H}, 8-\mathrm{H}), 3.39$ (dd, $J=7.5,2.5$ Hz, $1 \mathrm{H}, 1-\mathrm{H}), 3.44$ (dd, $J=11.5,6.8 \mathrm{~Hz}, 1 \mathrm{H}, 8$ '-H), 3.63 (dd, $J=10.4,1.5 \mathrm{~Hz}, 1 \mathrm{H}, 6-\mathrm{H}), 3.69-3.75$ (m, 4 H, 3-H, 4-H, 5-H, 6'-H), 4.47 (d, J=11.9 Hz, 1 H, CH $\left.2_{2}-\mathrm{Ph}\right), 4.52$ (d, J=11.9 Hz, $1 \mathrm{H}, \mathrm{CH}_{2^{-}}$ $\mathrm{Ph}), 4.57$ (d, $\left.J=11.4 \mathrm{~Hz}, 1 \mathrm{H}, \mathrm{CH}_{2}-\mathrm{Ph}\right), 4.69$ (d, $\left.J=11.9 \mathrm{~Hz}, 1 \mathrm{H}, \mathrm{CH}{ }_{2}-\mathrm{Ph}\right), 4.77$ (d, $J=11.9 \mathrm{~Hz}, 1$ $\left.\mathrm{H}, \mathrm{CH}_{2}-\mathrm{Ph}\right), 4.82\left(\mathrm{~d}, J=11.4 \mathrm{~Hz}, 1 \mathrm{H}, \mathrm{CH}_{2}-\mathrm{Ph}\right), 7.27-7.39(\mathrm{~m}, 15 \mathrm{H}$, arom. $\mathrm{H}) ;{ }^{13} \mathrm{C}$ NMR $(150$ $\mathrm{MHz}, \mathrm{CD}_{2} \mathrm{Cl}_{2}$ ): $\delta 19.4$ (d, C-2), 26.7 (d, C-7), 53.7 (d, C-1), 63.6 (t, C-8), 69.7 (t, C-6), 71.6, 73.6, $73.8\left(3 \mathrm{t}, \mathrm{CH}_{2} \mathrm{-Ph}\right), 73.9,75.0,76.8$ (3d, C-3, C-4, C-5), 127.8, 127.9, 128.0, 128.1, 128.2, 128.3, 128.4, 128.6, 128.7 (9d, C-arom.), 138.9, 139.0, 139.2 (3s, C-arom.); IR (film) $\tilde{v}=3416$ (OH), 3029, 2866, 1453, 1362, 1089, 1024, 735, $696 \mathrm{~cm}^{-1}$; HRMS (ESI-Q-TOF): $m / z$ calcd for $\mathrm{C}_{29} \mathrm{H}_{33} \mathrm{O}_{5}$ $[\mathrm{M}+\mathrm{H}]^{+}:$461.2328; found 461.2307; Elemental analysis: calcd (\%) for $\mathrm{C}_{29} \mathrm{H}_{32} \mathrm{O}_{5}$ (460.57): C 75.63, H 7.00; found: C 75.29, H 7.47.

\section{arabino-8:}

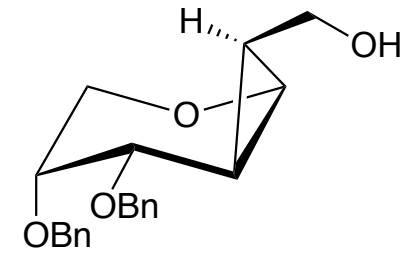

arabino-8

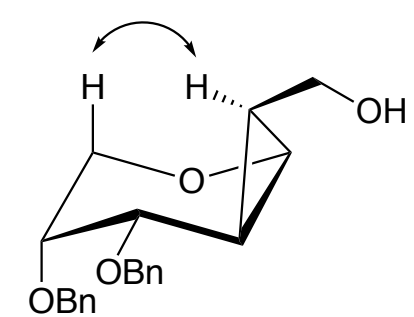

arabino-8

Colorless liquid $(1.65 \mathrm{~g}, 97 \%) . R_{\mathrm{f}}=0.22$ (hexane/ethyl acetate $\left.1: 1\right)$; $[\mathrm{a}]^{24}{ }_{\mathrm{D}}+15.0\left(\mathrm{c} 1.03, \mathrm{CHCl}_{3}\right)$; ${ }^{1} \mathrm{H}$ NMR (500 MHz, $\mathrm{CD}_{2} \mathrm{Cl}_{2}$ ): $\delta 1.06$ (dddd, $\left.J=7.4,6.6,6.2,2.1 \mathrm{~Hz}, 1 \mathrm{H}, 6-\mathrm{H}\right), 1.24$ (ddd, $J=6.9$, 6.2, $2.8 \mathrm{~Hz}, 1 \mathrm{H}, 2-\mathrm{H}), 1.76$ (bs, $1 \mathrm{H}, \mathrm{OH}), 3.34$ (dd, $J=11.6,7.4 \mathrm{~Hz}, 1 \mathrm{H}, 7-\mathrm{H}), 3.42$ (dd, $J=11.6$, $\left.6.6 \mathrm{~Hz}, 1 \mathrm{H}, 7^{\prime}-\mathrm{H}\right), 3.43$ (dd, $\left.J=6.9,2.1 \mathrm{~Hz}, 1 \mathrm{H}, 1-\mathrm{H}\right), 3.53$ (ddd, $\left.J=7.6,4.1,3.0 \mathrm{~Hz}, 1 \mathrm{H}, 4-\mathrm{H}\right)$, $3.56\left(\mathrm{dd}, J=10.3,4.1 \mathrm{~Hz}, 1 \mathrm{H}, 5-\mathrm{H}_{\text {equ. }}\right), 3.76\left(\mathrm{dd}, J=10.3,7.4 \mathrm{~Hz}, 1 \mathrm{H}, 5-\mathrm{H}_{\text {ax. }}\right), 4.03(\mathrm{dd}, J=3.0$, $2.8 \mathrm{~Hz}, 1 \mathrm{H}, 3-\mathrm{H}), 4.59$ (d, $\left.J=11.8 \mathrm{~Hz}, 1 \mathrm{H}, \mathrm{CH}_{2}-\mathrm{Ph}\right), 4.63$ (d, $\left.J=11.8 \mathrm{~Hz}, 1 \mathrm{H}, \mathrm{CH}_{2}-\mathrm{Ph}\right), 4.74$ (d, $J$ $\left.=12.3 \mathrm{~Hz}, 1 \mathrm{H}, \mathrm{CH}_{2} \mathrm{-Ph}\right), 4.76\left(\mathrm{~d}, J=12.3 \mathrm{~Hz}, 1 \mathrm{H}, \mathrm{CH}_{2}-\mathrm{Ph}\right), 7.28-7.42(\mathrm{~m}, 10 \mathrm{H}$, arom. $\mathrm{H}) ;{ }^{13} \mathrm{C}$ NMR (125 MHz, $\mathrm{CD}_{2} \mathrm{Cl}_{2}$ ): $\delta 21.6$ (d, C-2), 26.9 (d, C-6), 55.2 (d, C-1), 63.5 (t, C-7), 64.5 (t, C-5), 71.9, 72.4 (2t, $\left.\mathrm{CH}_{2}-\mathrm{Ph}\right), 73.8$ (d, C-3), 75.1 (d, C-4), 127.7, 127.8, 127.9, 128.1, 128.6, 128.7 (6d, C- 
arom.), 139.0, 139.3 (2s, C-arom.); IR (film) $\tilde{v}=3397$ (OH), 3028, 2872, 1454, 1206, 1112, 1024, 736, $697 \mathrm{~cm}^{-1}$; HRMS (ESI-Q-TOF): $\mathrm{m} / z$ calcd for $\mathrm{C}_{21} \mathrm{H}_{25} \mathrm{O}_{4}[\mathrm{M}+\mathrm{H}]^{+}: 341.1753$; found 341.1780; Elemental analysis: calcd (\%) for $\mathrm{C}_{21} \mathrm{H}_{24} \mathrm{O}_{4}$ (340.42): C 74.09, $\mathrm{H}$ 7.11; found: C 73.73, $\mathrm{H}$ 7.36.

xylo-8:

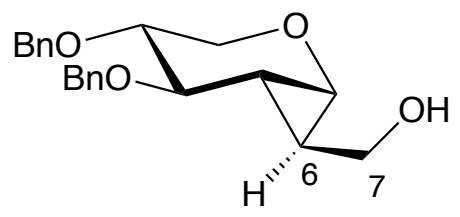

xylo-8

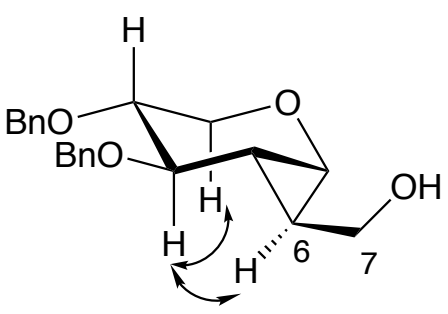

xylo-8

Colorless liquid (1.65 g, 97\%). $R_{\mathrm{f}}=0.29$ (hexane/ethyl acetate $\left.1: 1\right) ;[\mathrm{a}]^{21}{ }_{\mathrm{D}}-6.5\left(\mathrm{c} 0.97, \mathrm{CHCl}_{3}\right) ;{ }^{1} \mathrm{H}$ NMR (500 MHz, $\mathrm{CD}_{2} \mathrm{Cl}_{2}$ ): $\delta 1.03$ (dddd, $\left.J=7.1,6.0,1.7,1.0 \mathrm{~Hz}, 1 \mathrm{H}, 2-\mathrm{H}\right), 1.53$ (dddd, $J=7.4$, 6.7, 6.0, $2.2 \mathrm{~Hz}, 1 \mathrm{H}, 6-\mathrm{H}), 1.45$ (bs, $1 \mathrm{H}, \mathrm{OH}), 3.35$ (dd, $J=11.4,7.4 \mathrm{~Hz}, 1 \mathrm{H}, 7-\mathrm{H}), 3.43$ (dd, $J=$ 11.4, $\left.6.7 \mathrm{~Hz}, 1 \mathrm{H}, 7^{\prime}-\mathrm{H}\right), 3.46$ (ddd, $\left.J=7.1,2.2,0.6 \mathrm{~Hz}, 1 \mathrm{H}, 1-\mathrm{H}\right), 3.48$ (dddd, $J=3.4,3.2,2.3,1.0$ Hz, $1 \mathrm{H}, 4-\mathrm{H}), 3.63$ (ddd, $J=12.1,3.2,0.8 \mathrm{~Hz}, 1 \mathrm{H}, 5-\mathrm{H}), 3.68$ (dd, $J=12.1,2.3 \mathrm{~Hz}, 1 \mathrm{H}, 5$ '-H), 3.87 (dddd, $J=3.4,1.7,0.8,0.6 \mathrm{~Hz}, 1 \mathrm{H}, 3-\mathrm{H}), 4.51$ (d, $\left.J=11.7 \mathrm{~Hz}, 1 \mathrm{H}, \mathrm{CH}{ }_{2}-\mathrm{Ph}\right), 4.57(\mathrm{~d}, J=11.7 \mathrm{~Hz}$, $\left.1 \mathrm{H}, \mathrm{CH}_{2}-\mathrm{Ph}\right), 4.59\left(\mathrm{~d}, J=11.7 \mathrm{~Hz}, 1 \mathrm{H}, \mathrm{CH}_{2}-\mathrm{Ph}\right), 4.66\left(\mathrm{~d}, J=11.7 \mathrm{~Hz}, 1 \mathrm{H}, \mathrm{CH}_{2}-\mathrm{Ph}\right), 7.27-7.37(\mathrm{~m}$, $10 \mathrm{H}$, arom. H); ${ }^{13} \mathrm{C}$ NMR (125 MHz, $\mathrm{CD}_{2} \mathrm{Cl}_{2}$ ): $\delta 19.5$ (d, C-2), 25.4 (d, C-6), 55.7 (d, C-1), 63.7 (t, C-5), 63.8 (t, C-7), 71.5, 71.8 (2t, $\left.\mathrm{CH}_{2}-\mathrm{Ph}\right), 73.7$ (d, C-3), 76.0 (d, C-4), 128.0, 128.1, 128.2, 128.3, 128.7, 128.8 (6d, C-arom.), 138.9, 139.0 (2s, C-arom.); IR (film) $\tilde{v}=3419$ (OH), 3028, 2862, 1453, 1205, 1085, 1024, 736, $696 \mathrm{~cm}^{-1}$; HRMS (ESI-Q-TOF): $m / z$ calcd for $\mathrm{C}_{21} \mathrm{H}_{25} \mathrm{O}_{4}[\mathrm{M}+\mathrm{H}]^{+}: 341.1753$; found 341.1739; Elemental analysis: calcd (\%) for $\mathrm{C}_{21} \mathrm{H}_{24} \mathrm{O}_{4}$ (340.42): C 74.09, $\mathrm{H}$ 7.11; found: C 74.01, H 7.00.

\section{lyxo-8:}
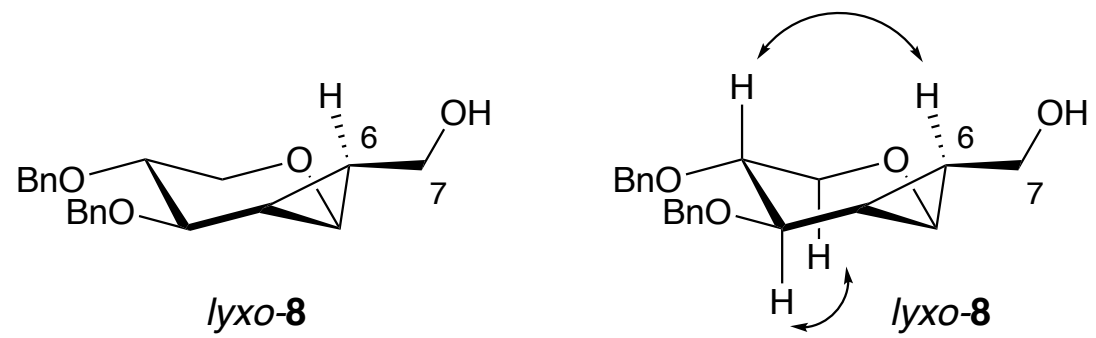

White solid (1.65 g, 97\%). $R_{\mathrm{f}}=0.29$ (hexane/ethyl acetate 1:1); $[\mathrm{a}]^{21} \mathrm{D}-101.2$ (c 0.78, $\left.\mathrm{CHCl}_{3}\right)$; m.p. 99-101 ${ }^{\circ} \mathrm{C} ;{ }^{1} \mathrm{H}$ NMR (500 MHz, $\left.\mathrm{CD}_{2} \mathrm{Cl}_{2}\right): \delta 1.35-1.40$ (m $\left.2 \mathrm{H}, 2-\mathrm{H}, 6-\mathrm{H}\right), 1.57$ (bs, $\left.1 \mathrm{H}, \mathrm{OH}\right), 3.12$ (t, $\left.J=10.6 \mathrm{~Hz}, 1 \mathrm{H}, 5-\mathrm{H}_{\text {ax. }}\right), 3.29-3.31(\mathrm{~m}, 1 \mathrm{H}, 7-\mathrm{H}), 3.34$ (ddd, $\left.J=10.6,7.1,4.2 \mathrm{~Hz}, 1 \mathrm{H}, 4-\mathrm{H}\right)$, $3.39\left(\mathrm{dd}, J=10.6,6.1 \mathrm{~Hz}, 1 \mathrm{H}, 7^{\prime}-\mathrm{H}\right), 3.60$ (dd, $\left.J=10.6,4.2 \mathrm{~Hz}, 1 \mathrm{H}, 5-\mathrm{H}_{\text {equ. }}\right), 3.63$ (dd, $J=6.9,2.7$ $\mathrm{Hz}, 1 \mathrm{H}, 1-\mathrm{H}), 4.05$ (dd, $J=7.1,6.8 \mathrm{~Hz}, 1 \mathrm{H}, 3-\mathrm{H}), 4.61\left(\mathrm{~d}, J=11.7 \mathrm{~Hz}, 1 \mathrm{H}, \mathrm{CH}_{2}-\mathrm{Ph}\right), 4.63(\mathrm{~d}, J=$ 
$\left.11.6 \mathrm{~Hz}, 1 \mathrm{H}, \mathrm{CH}_{2}-\mathrm{Ph}\right), 4.69$ (d, $\left.J=11.7 \mathrm{~Hz}, 1 \mathrm{H}, \mathrm{CH}_{2}-\mathrm{Ph}\right), 4.81$ (d, $J=11.6 \mathrm{~Hz}, 1 \mathrm{H}, \mathrm{CH}_{2}-\mathrm{Ph}$ ), 7.24-7.40 (m, $10 \mathrm{H}$, arom. H); ${ }^{13} \mathrm{C}$ NMR (125 MHz, $\left.\mathrm{CD}_{2} \mathrm{Cl}_{2}\right): \delta 21.2$ (d, C-2), 7.44 (d, C-6), 60.0 (d, C-1), 63.6 (t, C-7), 66.5 (t, C-5), 70.8, 72.9 (2t, $\left.\mathrm{CH}_{2}-\mathrm{Ph}\right), 77.2$ (d, C-3), 78.2 (d, C-4), 127.9, 128.0, 128.1, 128.3, 128.7, 128.8 (6d, C-arom.), 139.2, 139.3 (2s, C-arom.); IR (film) $\tilde{v}=3430$ (OH), 3028, 2865, 1453, 1205, 1092, 1067, 1025, 736, $697 \mathrm{~cm}^{-1}$; HRMS (ESI-Q-TOF): $\mathrm{m} / z$ calcd for $\mathrm{C}_{21} \mathrm{H}_{24} \mathrm{NaO}_{4}[\mathrm{M}+\mathrm{Na}]^{+}:$363.1572; found 363.1576; Elemental analysis: calcd (\%) for $\mathrm{C}_{21} \mathrm{H}_{24} \mathrm{O}_{4}$ (340.42): C 74.09, H 7.11; found: C 74.02, H 7.12.

\section{Separation of alcohols $x y l o-8$ and $l y x o-8$}

A 75:25 mixture of alcohols $x y l o-8$ and $l y x o-8(3.4 \mathrm{~g}, 10 \mathrm{mmol})$, obtained from direct reduction of a 75:25 mixture of esters xylo/lyxo-7 (see Chapter 3), was dissolved in $120 \mathrm{~mL}$ of dry dichloromethane and cooled to $0^{\circ} \mathrm{C}$. Triethylamine $(2.03 \mathrm{~g}, 2.8 \mathrm{~mL}, 20 \mathrm{mmol})$, DMAP (122 mg, $\left.1 \mathrm{mmol}\right)$, and 3,5dinitrobenzoyl chloride $(3.5 \mathrm{~g}, 15 \mathrm{mmol})$ were added successively at this temperature. The reaction was stirred until TLC showed complete conversion $(3 \mathrm{~h})$ of the starting material and meanwhile allowed to warm to room temperature. The reaction was diluted with dichloromethane $(50 \mathrm{~mL})$ and consecutively washed with $1 \mathrm{M} \mathrm{HCl}$, sat. $\mathrm{NaHCO}_{3}$ solution, and brine (each 1 x $50 \mathrm{~mL}$ ). After drying over $\mathrm{Na}_{2} \mathrm{SO}_{4}$ and removing of the solvent, the crude product ( $5.5 \mathrm{~g}$ ) was purified by column chromatography with hexane / methyl-tert.butyl-ether (80:20). Pure xylo-benzoate (2.2 g, 41\%), a small amount of lyxo-benzoate $(420 \mathrm{mg}, 8 \%)$, and a mixture fraction $(2.4 \mathrm{~g}, 45 \%)$ was isolated.

The xylo benzoate $(1.07 \mathrm{~g}, 2 \mathrm{mmol})$ was dissolved in a mixture of dry dichloromethane $(20 \mathrm{~mL})$ and dry methanol $(20 \mathrm{~mL})$ and $\mathrm{K}_{2} \mathrm{CO}_{3}(28 \mathrm{mg}, 0.2 \mathrm{mmol})$ was added at room temperature. When TLC showed complete cleavage of the benzoate $(2 \mathrm{~h})$, the solvent was removed and the crude alcohol was purified by column chromatography using hexane / methyl-tert.butyl-ether (40:60) to afford pure alcohol xylo-8 (650 mg, 95\%) (for analytical data see Chapter 3).

xylo-benzoate:
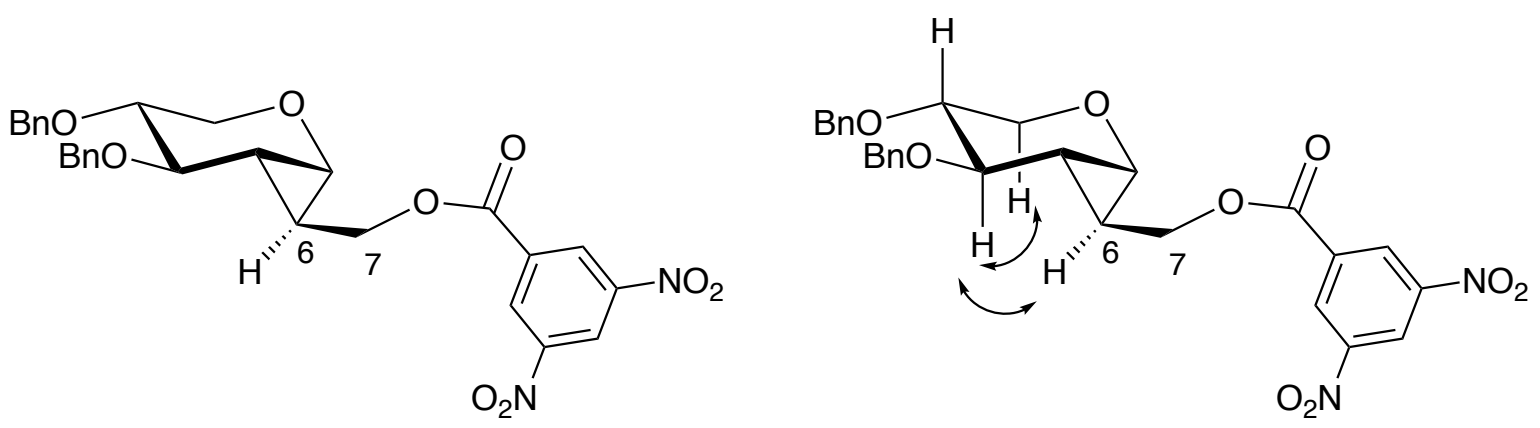
Yellow syrup. $R_{\mathrm{f}}=0.28$ (hexane/ethyl acetate $\left.8: 2\right) ;[\mathrm{a}]^{26}{ }_{\mathrm{D}}+8.0\left(\mathrm{c} 1.01, \mathrm{CHCl}_{3}\right) ;{ }^{1} \mathrm{H}$ NMR $(500$ $\mathrm{MHz}, \mathrm{CD}_{2} \mathrm{Cl}_{2}$ ): $\delta 1.24$ (dddd, $\left.J=7.2,6.0,2.2,1.0 \mathrm{~Hz}, 1 \mathrm{H}, 2-\mathrm{H}\right), 1.84$ (dddd, $J=8.2,7.1,6.0,2.2$ $\mathrm{Hz}, 1 \mathrm{H}, 6-\mathrm{H}), 3.51$ (dddd, $J=3.3,2.0,1.4,1.0 \mathrm{~Hz}, 1 \mathrm{H}, 4-\mathrm{H}), 3.66$ (ddd, $J=12.1,1.4 \mathrm{~Hz}, 1 \mathrm{H}, 5-$ H), $3.68(\mathrm{ddd}, J=7.2,2.2,1.0 \mathrm{~Hz}, 1 \mathrm{H}, 1-\mathrm{H}), 3.71(\mathrm{dd}, J=12.1,2.0 \mathrm{~Hz}, 1 \mathrm{H}, 5$ '-H), 3.93 (ddd, $J=$ 3.3, 2.2, $1.0 \mathrm{~Hz}, 1 \mathrm{H}, 3-\mathrm{H}), 4.19$ (dd, $J=11.7,8.2 \mathrm{~Hz}, 1 \mathrm{H}, 7-\mathrm{H}), 4.33$ (dd, $J=11.7,7.1 \mathrm{~Hz}, 1 \mathrm{H}, 7^{\prime}-$ $\mathrm{H}), 4.51\left(\mathrm{~d}, J=11.8 \mathrm{~Hz}, 1 \mathrm{H}, \mathrm{CH}_{2}-\mathrm{Ph}\right), 4.58\left(\mathrm{~d}, J=11.8 \mathrm{~Hz}, 1 \mathrm{H}, \mathrm{CH}_{2}-\mathrm{Ph}\right), 4.60(\mathrm{~d}, J=11.8 \mathrm{~Hz}, 1$ $\left.\mathrm{H}, \mathrm{CH}_{2}-\mathrm{Ph}\right), 4.65$ (d, $\left.J=11.8 \mathrm{~Hz}, 1 \mathrm{H}, \mathrm{CH}_{2}-\mathrm{Ph}\right), 7.26-7.36(\mathrm{~m}, 10 \mathrm{H}$, arom. H), 9.15 (d, $J=2.1 \mathrm{~Hz}$, $2 \mathrm{H}, o$-benzoate), $9.21\left(\mathrm{t}, J=2.1 \mathrm{~Hz}, 1 \mathrm{H}, p\right.$-benzoate); ${ }^{13} \mathrm{C} \mathrm{NMR}\left(125 \mathrm{MHz}, \mathrm{CD}_{2} \mathrm{Cl}_{2}\right): \delta 20.5(\mathrm{~d}, \mathrm{C}-$ 2), 21.6 (d, C-6), 56.1 (d, C-1), 63.4 (t, C-5), 68.2 (t, C-7), 71.5, 71.9 (2t, $\left.\mathrm{CH}_{2}-\mathrm{Ph}\right), 72.7$ (d, C-3), 75.6 (d, C-4), 122.8 (d, p-benzoate), 128.0, 128.1, 128.2, 128.6, 128.8, 128.9 (6d, C-arom.), 129.9 (d, o-benzoate), 134.5 (s, ipso-benzoate), 138.7, 138.8 (2s, C-arom.), 149.2 (s, $m$-benzoate), 163.1 (s, COAr); IR (film) $\tilde{v}=3098,3025,2869,1727,1541,1455,1343,1273,1162,1069,918,722,697$ $\mathrm{cm}^{-1}$; HRMS (ESI-Q-TOF): $m / z$ calcd for $\mathrm{C}_{28} \mathrm{H}_{27} \mathrm{~N}_{2} \mathrm{O}_{9}[\mathrm{M}+\mathrm{H}]^{+}: 535.1717$; found 535.1747.

\section{lyxo-benzoate:}
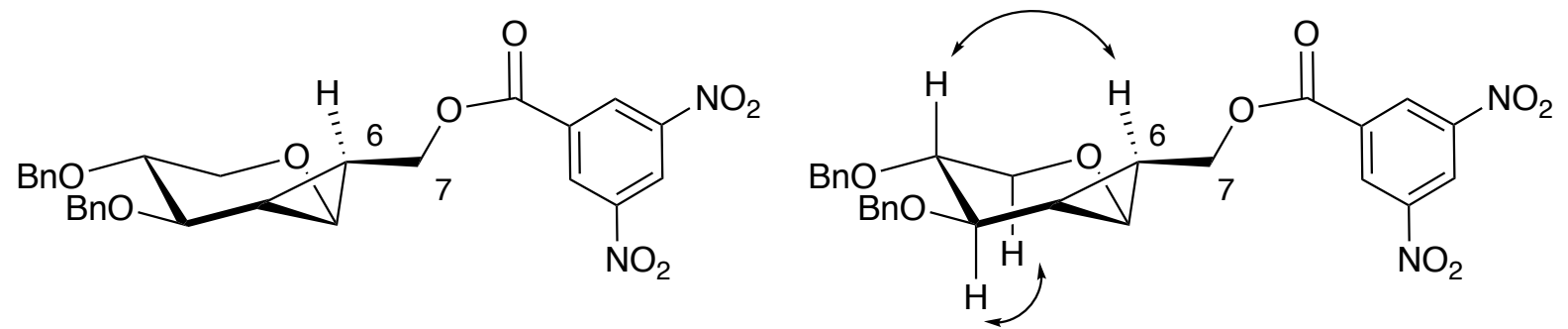

Yellow syrup. $R_{\mathrm{f}}=0.22$ (hexane/ethyl acetate 8:2); $[\mathrm{a}]^{21}{ }_{\mathrm{D}}-62.8\left(\mathrm{c} 0.99, \mathrm{CHCl}_{3}\right) ;{ }^{1} \mathrm{H}$ NMR $(500$ $\left.\mathrm{MHz}, \mathrm{CD}_{2} \mathrm{Cl}_{2}\right): \delta 1.65(\mathrm{td}, J=7.1,6.2 \mathrm{~Hz}, 1 \mathrm{H}, 2-\mathrm{H}), 1.70$ (dddd, $\left.J=8.6,6.7,6.2,2.4 \mathrm{~Hz}, 1 \mathrm{H}, 6-\mathrm{H}\right)$, $3.18\left(\mathrm{t}, J=10.7 \mathrm{~Hz}, 1 \mathrm{H}, 5-\mathrm{H}_{\text {ax. }}\right), 3.38$ (ddd, $\left.J=10.7,7.1,4.1 \mathrm{~Hz}, 1 \mathrm{H}, 4-\mathrm{H}\right), 3.63$ (dd, $J=10.7,4.1$ Hz, $1 \mathrm{H}, 5$ '- $\mathrm{H}_{\text {equ. }}$ ), 3.82 (dd, $\left.J=7.1,2.4 \mathrm{~Hz}, 1 \mathrm{H}, 1-\mathrm{H}\right), 4.06$ (dd, $\left.J=11.7,8.6 \mathrm{~Hz}, 1 \mathrm{H}, 7-\mathrm{H}\right), 4.07$ (t, $J=7.1 \mathrm{~Hz}, 1 \mathrm{H}, 3-\mathrm{H}), 4.50\left(\mathrm{dd}, J=11.7,6.7 \mathrm{~Hz}, 1 \mathrm{H}, 7^{\prime}-\mathrm{H}\right), 4.57\left(\mathrm{~d}, J=11.9 \mathrm{~Hz}, 1 \mathrm{H}, \mathrm{CH}_{2}-\mathrm{Ph}\right)$, $4.60\left(\mathrm{~d}, J=11.8 \mathrm{~Hz}, 1 \mathrm{H}, \mathrm{CH}_{2}-\mathrm{Ph}\right), 4.67$ (d, $\left.J=11.8 \mathrm{~Hz}, 1 \mathrm{H}, \mathrm{CH}_{2}-\mathrm{Ph}\right), 4.75$ (d, $J=11.9 \mathrm{~Hz}, 1 \mathrm{H}$, $\left.\mathrm{CH}_{2} \mathrm{Ph}\right), 7.12-7.33(\mathrm{~m}, 10 \mathrm{H}$, arom. $\mathrm{H}), 9.00$ (d, $J=2.1 \mathrm{~Hz}, 2 \mathrm{H}, o$-benzoate), 9.10 (t, $J=2.1 \mathrm{~Hz}, 1$ $\mathrm{H}, p$-benzoate); ${ }^{13} \mathrm{C}$ NMR (125 MHz, $\mathrm{CD}_{2} \mathrm{Cl}_{2}$ ): $\delta 22.5$ (d, C-2), 24.1 (d, C-6), $60.2(\mathrm{~d}, \mathrm{C}-1), 66.6(\mathrm{t}$, C-5), 67.9 (t, C-7), 70.5, 73.0 (2t, $\left.\mathrm{CH}_{2}-\mathrm{Ph}\right), 76.9$ (d, C-3), 77.9 (d, C-4), 122.7 (d, p-benzoate), 127.3, 127.7, 128.0, 128.1, 128.5, 128.7 (6d, C-arom.), 129.7 (d, o-benzoate), 134.2 (s, ipso-benzoate), 139.0, 139.1 (2s, C-arom.), 149.0 (s, $m$-benzoate), 163.0 (s, COAr); IR (film) $\tilde{v}=3660,3099,2978$, 2879, 1728, 1629, 1541, 1455, 1343, 1272, 1161, 1071, 975, 918, 722, $697 \mathrm{~cm}^{-1}$; HRMS (ESI-QTOF): $m / z$ calcd for $\mathrm{C}_{28} \mathrm{H}_{27} \mathrm{~N}_{2} \mathrm{O}_{9}[\mathrm{M}+\mathrm{H}]^{+}:$535.1717; found 535.1689. 


\section{General procedure for synthesis of xanthates 9}

Alcohol $8(1 \mathrm{mmol})$ was dissolved in dry tetrahydrofuran $(6 \mathrm{~mL})$ under an argon atmosphere at room temperature. Sodium hydride (102 $\mathrm{mg}, 4 \mathrm{mmol})$ and carbon disulfide $(0.18 \mathrm{~mL}, 3 \mathrm{mmol})$ were added successively at this temperature. After stirring for $1 \mathrm{~h}$, iodomethane $(0.375 \mathrm{~mL}, 6 \mathrm{mmol})$ was added and stirring was continued until TLC showed complete conversion (10 to $45 \mathrm{~min}$ ). The reaction was quenched with ice $(40 \mathrm{~mL})$ and extracted with dichloromethane $(4 \times 25 \mathrm{~mL})$. Prior to drying with $\mathrm{Na}_{2} \mathrm{SO}_{4}$ the combined organic layers were washed with water $(20 \mathrm{~mL})$ and brine $(20 \mathrm{~mL})$. Removal of the solvent at $40{ }^{\circ} \mathrm{C}$ afforded a pale yellow oil in quantitative yield, which was directly submitted to NMR, other analytical data were obtained from the crude products as well. The xanthates 9 were directly used for the radical reactions without further purification.

gluco-9:
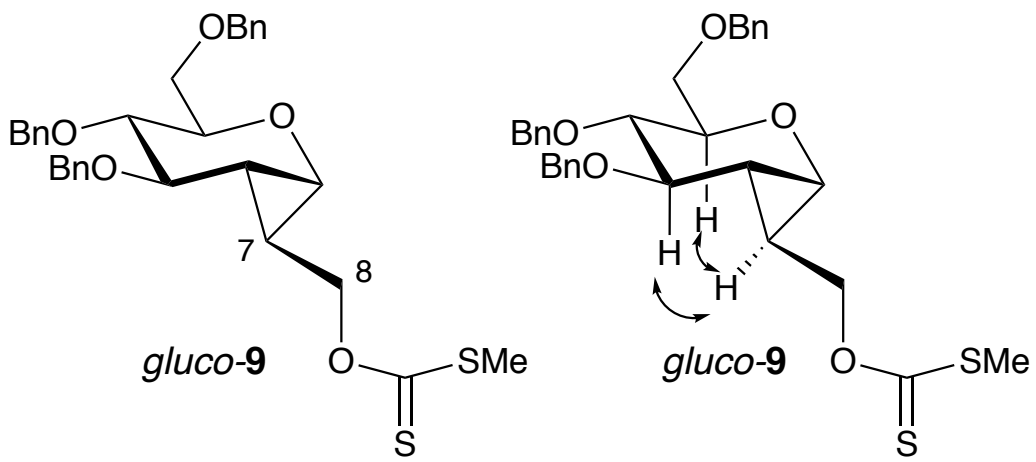

Pale yellow syrup (crude $550 \mathrm{mg},>98 \%$ ). $R_{\mathrm{f}}=0.47$ (hexane/ethyl acetate 8:2); $[\mathrm{a}]^{24}{ }_{\mathrm{D}}+36.3(\mathrm{c} 1.01$, $\left.\mathrm{CHCl}_{3}\right) ;{ }^{1} \mathrm{H} \mathrm{NMR}\left(600 \mathrm{MHz}, \mathrm{CD}_{2} \mathrm{Cl}_{2}\right): \delta 1.13$ (ddd, $\left.J=7.2,5.5,3.5 \mathrm{~Hz}, 1 \mathrm{H}, 2-\mathrm{H}\right), 1.61$ (dddd, $J=$ 7.9, 7.4, 5.5, 2.2 Hz, 1 H, 7-H), 2.57 (s, 3 H, SCH 3 ), 3.55 (dd, $J=10.4,3.5$ Hz, 1 H, 6-H), 3.56 (dd, $J=7.2,5.9 \mathrm{~Hz}, 1 \mathrm{H}, 4-\mathrm{H}), 3.57$ (dd, $J=7.2,2.2 \mathrm{~Hz}, 1 \mathrm{H}, 1-\mathrm{H}), 3.66$ (dd, $J=7.2,3.5 \mathrm{~Hz}, 1 \mathrm{H}, 3-\mathrm{H})$, $3.70(\mathrm{dd}, J=10.4,6.1 \mathrm{~Hz}, 1 \mathrm{H}, 6$ '-H), 3.75 (ddd $J=6.1,5.9,3.5 \mathrm{~Hz}, 1 \mathrm{H}, 5-\mathrm{H}), 4.38$ (dd, $J=11.7$, $7.9 \mathrm{~Hz}, 1 \mathrm{H}, 8-\mathrm{H}), 4.50$ (d, $\left.J=12.0 \mathrm{~Hz}, 1 \mathrm{H}, \mathrm{CH}_{2}-\mathrm{Ph}\right), 4.54$ (d, $\left.J=12.0 \mathrm{~Hz}, 1 \mathrm{H}, \mathrm{CH}_{2}-\mathrm{Ph}\right), 4.54$ (dd, $\left.J=11.7,7.4 \mathrm{~Hz}, 1 \mathrm{H}, 8^{\prime}-\mathrm{H}\right), 4.57$ (d, $\left.J=11.5 \mathrm{~Hz}, 1 \mathrm{H}, \mathrm{CH}_{2}-\mathrm{Ph}\right), 4.63$ (d, J=11.9 Hz, $\left.1 \mathrm{H}, \mathrm{CH}_{2}-\mathrm{Ph}\right)$, $4.78\left(\mathrm{~d}, J=11.5 \mathrm{~Hz}, 1 \mathrm{H}, \mathrm{CH}_{2}-\mathrm{Ph}\right), 4.79$ (d, $\left.J=11.9 \mathrm{~Hz}, 1 \mathrm{H}, \mathrm{CH}_{2}-\mathrm{Ph}\right), 7.28-7.36$ (m, $15 \mathrm{H}$, arom. $\mathrm{H}) ;{ }^{13} \mathrm{C}$ NMR $\left(150 \mathrm{MHz}, \mathrm{CD}_{2} \mathrm{Cl}_{2}\right): \delta 19.4\left(\mathrm{q}, \mathrm{SCH}_{3}\right), 21.0(\mathrm{~d}, \mathrm{C}-2), 23.2$ (d, C-7), $54.4(\mathrm{~d}, \mathrm{C}-1), 70.4$ (t, C-6), 71.3, 73.6, 73.7 (3t, $\left.\mathrm{CH}_{2}-\mathrm{Ph}\right), 74.6$ (t, C-8), 76.7 (d, C-4), 77.2 (d, C-5), 78.5 (d, C-3), 127.9, 128.0, 128.1, 128.2, 128.3, 128.5, 128.6, 128.7, 128.8 (9d, C-arom.), 138.7, 138.8, 139.0 (3s, Carom.), 216.5 (s, OCS); IR (film) $\tilde{v}=3029,2924,2866,1648,1453,1208,1056,734,697 \mathrm{~cm}^{-1}$; HRMS (ESI-Q-TOF): $m / z$ calcd for $\mathrm{C}_{31} \mathrm{H}_{35} \mathrm{~S}_{2} \mathrm{O}_{5}[\mathrm{M}+\mathrm{H}]^{+}:$: 551.1926; found 551.1948. 


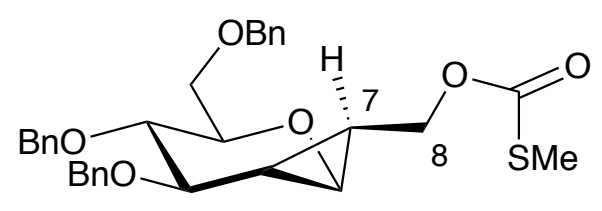

manno-9

Pale yellow syrup (crude $550 \mathrm{mg},>98 \%$ ). $R_{\mathrm{f}}=0.26$ (hexane/ethyl acetate 8:2); $[\mathrm{a}]^{23}{ }_{\mathrm{D}}-31.0(\mathrm{c} 1.00$, $\mathrm{CHCl}_{3}$ ); ${ }^{1} \mathrm{H}$ NMR (600 MHz, $\left.\mathrm{CD}_{2} \mathrm{Cl}_{2}\right): \delta 1.58$ (td, $\left.J=7.1,6.1 \mathrm{~Hz}, 1 \mathrm{H}, 2-\mathrm{H}\right), 1.69$ (ddddd, $J=8.3$, 6.9, 6.1, 2.2 Hz, $1 \mathrm{H}, 7-\mathrm{H}), 2.53$ (s, $3 \mathrm{H}, \mathrm{SCH}_{3}$ ), 3.34 (dd, $\left.J=10.0,6.9 \mathrm{~Hz}, 1 \mathrm{H}, 4-\mathrm{H}\right), 3.42$ (ddd, $J=$ 10.0, 5.0, 1.9 Hz, 1 H, 5-H), 3.56 (dd, $J=10.7,5.0$ Hz, 1 H, 6-H), 3.69 (dd, $J=10.7,1.9 \mathrm{~Hz}, 1 \mathrm{H}, 6$ 'H), $3.81(\mathrm{dd}, J=7.1,2.2 \mathrm{~Hz}, 1 \mathrm{H}, 1-\mathrm{H}), 4.17$ (dd, $J=7.1,6.9 \mathrm{~Hz}, 1 \mathrm{H}, 3-\mathrm{H}), 4.30$ (dd, $J=11.6,8.3$ $\mathrm{Hz}, 1 \mathrm{H}, 8-\mathrm{H}), 4.49$ (d, $\left.J=11.8 \mathrm{~Hz}, 1 \mathrm{H}, \mathrm{CH}_{2}-\mathrm{Ph}\right), 4.52$ (d, $\left.J=11.8 \mathrm{~Hz}, 1 \mathrm{H}, \mathrm{CH}_{2}-\mathrm{Ph}\right), 4.55(\mathrm{~d}, J=$ $\left.11.2 \mathrm{~Hz}, 1 \mathrm{H}, \mathrm{CH}_{2}-\mathrm{Ph}\right), 4.57$ (d, $\left.J=11.4 \mathrm{~Hz}, 1 \mathrm{H}, \mathrm{CH}_{2}-\mathrm{Ph}\right), 4.63$ (dd, $\left.J=11.6,6.9 \mathrm{~Hz}, 1 \mathrm{H}, 8^{\prime}-\mathrm{H}\right)$, $4.81\left(\mathrm{~d}, J=11.2 \mathrm{~Hz}, 1 \mathrm{H}, \mathrm{CH}_{2}-\mathrm{Ph}\right), 4.84$ (d, $\left.J=11.4 \mathrm{~Hz}, 1 \mathrm{H}, \mathrm{CH}_{2}-\mathrm{Ph}\right), 7.20-7.42$ (m, $15 \mathrm{H}$, arom. $\mathrm{H}) ;{ }^{13} \mathrm{C}$ NMR (150 MHz, $\mathrm{CD}_{2} \mathrm{Cl}_{2}$ ): $\delta 19.4$ (q, $\left.\mathrm{SCH}_{3}\right), 22.2$ (d, C-2), 23.8 (d, C-7), 59.6 (d, C-1), 69.8 (t, C-6), 70.4, 73.8, 74.5 (3t, $\left.\mathrm{CH}_{2}-\mathrm{Ph}\right), 74.7$ (t, C-8), 77.8 (d, C-5), 78.4 (d, C-3), 78.6 (d, C-4), 127.8, $127.9,128.0,128.1,128.2,128.4,128.5,128.6,128.7$ (9d, C-arom.), 138.8, 139.0, 139.1 (3s, Carom.); 216.7 (s, OCS); IR (solution) $\tilde{v}=3028,2921,2865,1495,1452,1364,1208,1061,1027$, 909, 736, $698 \mathrm{~cm}^{-1}$; HRMS (ESI-Q-TOF): $\mathrm{m} / \mathrm{z}$ calcd for $\mathrm{C}_{31} \mathrm{H}_{35} \mathrm{~S}_{2} \mathrm{O}_{5}[\mathrm{M}+\mathrm{H}]^{+}:$551.1926; found 551.1906.

\section{galacto-9:}
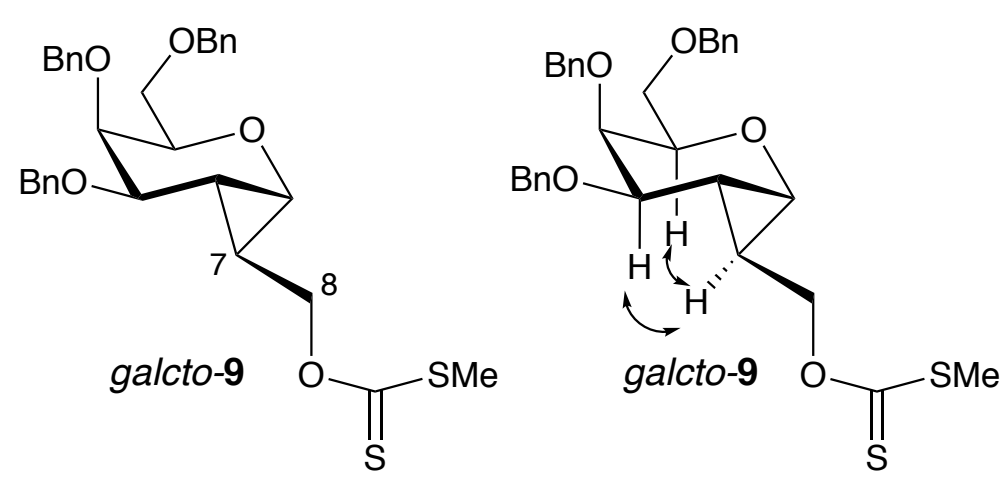

Pale yellow syrup (crude $550 \mathrm{mg},>98 \%$ ). $R_{\mathrm{f}}=0.58$ (hexane/ethyl acetate 8:2); $[\mathrm{a}]^{24}{ }_{\mathrm{D}}+28.5$ (c 1.00, $\left.\mathrm{CHCl}_{3}\right) ;{ }^{1} \mathrm{H}$ NMR (500 MHz, $\mathrm{CD}_{2} \mathrm{Cl}_{2}$ ): $\delta 1.33-1.40(\mathrm{~m}, 2 \mathrm{H}, 2-\mathrm{H}, 7-\mathrm{H}), 2.60\left(\mathrm{~s}, 3 \mathrm{H}, \mathrm{SCH}_{3}\right), 3.61$ (dd, $J=7.1,2.5 \mathrm{~Hz}, 1 \mathrm{H}, 1-\mathrm{H}), 3.67-3.77$ (m, $5 \mathrm{H}, 3-\mathrm{H}, 4-\mathrm{H}, 5-\mathrm{H}, 6-\mathrm{H}), 4.43$ (dd, $J=11.5,7.4 \mathrm{~Hz}, 1$ $\mathrm{H}, 8-\mathrm{H}), 4.52$ (d, $\left.J=11.8 \mathrm{~Hz}, 1 \mathrm{H}, \mathrm{CH}_{2}-\mathrm{Ph}\right), 4.57$ (d, $\left.J=11.8 \mathrm{~Hz}, 1 \mathrm{H}, \mathrm{CH}_{2}-\mathrm{Ph}\right), 4.61$ (d, $J=11.4$ $\left.\mathrm{Hz}, 1 \mathrm{H}, \mathrm{CH}_{2}-\mathrm{Ph}\right), 4.63$ (dd, $\left.J=11.5,3.1 \mathrm{~Hz}, 1 \mathrm{H}, 8^{\prime}-\mathrm{H}\right), 4.72$ (d, J=12.0 Hz, $\left.1 \mathrm{H}, \mathrm{CH}_{2}-\mathrm{Ph}\right), 4.86$ (d, $\left.J=12.0 \mathrm{~Hz}, 1 \mathrm{H}, \mathrm{CH}_{2}-\mathrm{Ph}\right), 4.90\left(\mathrm{~d}, J=11.4 \mathrm{~Hz}, 1 \mathrm{H}, \mathrm{CH}_{2}-\mathrm{Ph}\right), 7.33-7.46$ (m, $15 \mathrm{H}$, arom. $\left.\mathrm{H}\right) ;{ }^{13} \mathrm{C}$ NMR (125 MHz, $\mathrm{CD}_{2} \mathrm{Cl}_{2}$ ): $\delta 19.4$ (q, $\left.\mathrm{SCH}_{3}\right), 20.0$ (d, C-2), 22.8 (d, C-7), 54.4 (d, C-1), 69.7 (t, C-6), 71.3, 73.6, 74.1 (3t, $\left.\mathrm{CH}_{2}-\mathrm{Ph}\right), 73.6,75.4,77.0$ (3d, C-3, C-4, C-5), 75.0 (t, C-8), 127.7, 127.8, 127.9, 
128.0, 128.1, 128.2, 128.6, 128.7, 128.8 (9d, C-arom.), 138.8, 138.9, 139.2 (3s, C-arom.), 216.5 (s, OCS); IR (film) $\tilde{v}=2919,2864,1453,1204,1089,1053,735,696 \mathrm{~cm}^{-1}$; HRMS (ESI-Q-TOF): $\mathrm{m} / \mathrm{z}$ calcd for $\mathrm{C}_{31} \mathrm{H}_{35} \mathrm{~S}_{2} \mathrm{O}_{5}[\mathrm{M}+\mathrm{H}]^{+}:$551.1926; found 551.1943.

\section{arabino-9:}

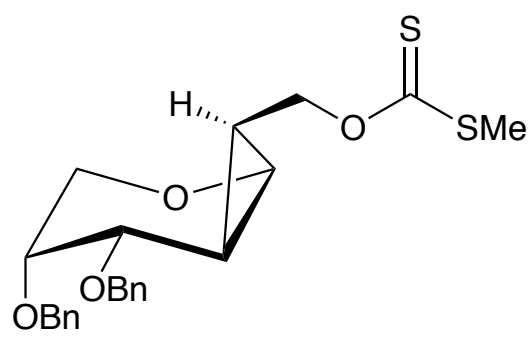

arabino-9

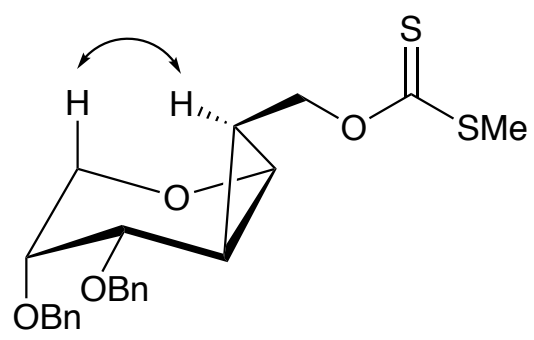

arabino-9

Pale yellow syrup (crude $430 \mathrm{mg},>98 \%$ ). $R_{\mathrm{f}}=0.26$ (hexane/ethyl acetate 8:2) $[\mathrm{a}]^{23} \mathrm{D}+11.7(\mathrm{c} 1.00$, $\mathrm{CHCl}_{3}$ ); ${ }^{1} \mathrm{H} \mathrm{NMR}\left(500 \mathrm{MHz}, \mathrm{CD}_{2} \mathrm{Cl}_{2}\right.$ ): $\delta 1.31$ (dddd, $\left.J=7.6,7.5,6.1,2.1 \mathrm{~Hz}, 1 \mathrm{H}, 6-\mathrm{H}\right), 1.40$ (ddd, $J=7.1,6.1,2.9 \mathrm{~Hz}, 1 \mathrm{H}, 2-\mathrm{H}), 2.56$ (s, $3 \mathrm{H}, \mathrm{SCH}_{3}$ ), 3.56 (ddd, $\left.J=7.3,4.1,3.0 \mathrm{~Hz}, 1 \mathrm{H}, 4-\mathrm{H}\right), 3.57$ (dd, $J=7.1,2.1 \mathrm{~Hz}, 1 \mathrm{H}, 1-\mathrm{H}), 3.58$ (dd, $\left.J=10.6,4.1 \mathrm{~Hz}, 1 \mathrm{H}, 5-\mathrm{H}_{\text {equ. }}\right), 3.78$ (dd, $J=10.6,7.3 \mathrm{~Hz}, 1$ H, 5- $\mathrm{H}_{\mathrm{ax} .}$ ), 3.99 (dd, $\left.J=3.0,2.9 \mathrm{~Hz}, 1 \mathrm{H}, 3-\mathrm{H}\right), 4.40(\mathrm{dd}, J=11.6,7.5 \mathrm{~Hz}, 1 \mathrm{H}, 7-\mathrm{H}), 4.46(\mathrm{dd}, J=$ 11.6, 7.6 Hz, $\left.1 \mathrm{H}, 7^{\prime}-\mathrm{H}\right), 4.60$ (d, $\left.J=11.8 \mathrm{~Hz}, 1 \mathrm{H}, \mathrm{CH}_{2}-\mathrm{Ph}\right), 4.65$ (d, J=11.8 Hz, $\left.1 \mathrm{H}, \mathrm{CH}_{2}-\mathrm{Ph}\right)$, $4.73\left(\mathrm{~d}, J=11.9 \mathrm{~Hz}, 1 \mathrm{H}, \mathrm{CH}_{2}-\mathrm{Ph}\right), 4.78\left(\mathrm{~d}, J=11.9 \mathrm{~Hz}, 1 \mathrm{H}, \mathrm{CH}_{2}-\mathrm{Ph}\right), 7.34-7.36(\mathrm{~m}, 10 \mathrm{H}$, arom. $\mathrm{H}) ;{ }^{13} \mathrm{C}$ NMR (125 MHz, $\mathrm{CD}_{2} \mathrm{Cl}_{2}$ ): $\delta 19.3\left(\mathrm{q}, \mathrm{SCH}_{3}\right), 22.2$ (d, C-2), 22.9 (d, C-6), 55.4 (d, C-1), 65.0 (t, C-5), 72.0, 72.2 (2t, $\left.\mathrm{CH}_{2}-\mathrm{Ph}\right), 73.6$ (d, C-3), 74.8 (t, C-7), 74.9 (d, C-4), 127.7, 127.8, 127.9, 128.2, 128.6, 128.7 (6d, C-arom.), 139.0, 139.1 (2s, C-arom.), 216.4 (s, OCS); IR (film) $\tilde{v}=3028$, 2922, 2874, 1453, 1202, 1113, 1050, 736, $696 \mathrm{~cm}^{-1}$; HRMS (ESI-Q-TOF): $m / z$ calcd for $\mathrm{C}_{23} \mathrm{H}_{27} \mathrm{~S}_{2} \mathrm{O}_{4}$ $[\mathrm{M}+\mathrm{H}]^{+}: 431.1351$; found 431.1309 .

xylo-9:

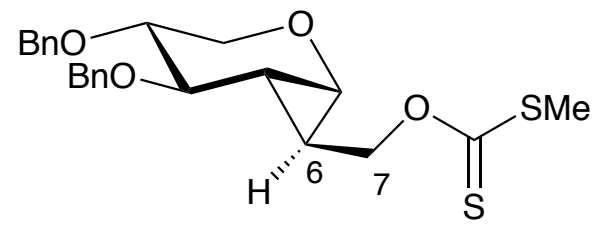

xylo-9

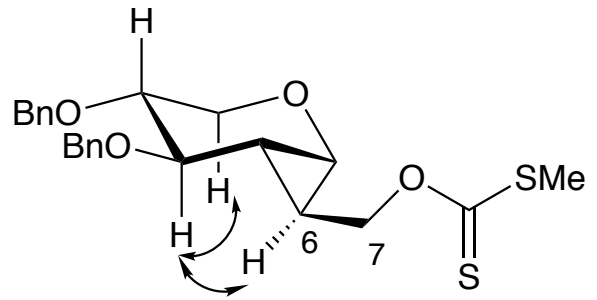

xylo-9

Pale yellow syrup (crude $430 \mathrm{mg},>98 \%) . R_{\mathrm{f}}=0.31$ (hexane/ethyl acetate 8:2); $[\alpha]^{21} \mathrm{D}-6.4(\mathrm{c} 1.01$, $\mathrm{CHCl}_{3}$ ); ${ }^{1} \mathrm{H}$ NMR (500 MHz, $\mathrm{CD}_{2} \mathrm{Cl}_{2}$ ): $\delta 1.20$ (dddd, $\left.J=7.2,6.0,1.6,1.2 \mathrm{~Hz}, 1 \mathrm{H}, 2-\mathrm{H}\right), 1.81$ (tddd, $J=7.6,6.0,2.2,0.9 \mathrm{~Hz}, 1 \mathrm{H}, 6-\mathrm{H}), 2.56$ (s, $3 \mathrm{H}, \mathrm{SCH}_{3}$ ), 3.49 (dddd, $J=3.4,3.1,2.1,1.2 \mathrm{~Hz}, 1 \mathrm{H}, 4-$ H), $3.60(\mathrm{ddd}, J=7.2,2.2,0.9 \mathrm{~Hz}, 1 \mathrm{H}, 1-\mathrm{H}), 3.65(\mathrm{ddd}, J=12.1,3.1 \mathrm{~Hz}, 1 \mathrm{H}, 5-\mathrm{H}), 3.70(\mathrm{dd}, J=$ 12.1, 2.1 Hz, $1 \mathrm{H}, 5$ '-H), 3.89 (ddd, $J=3.4,1.6,0.9 \mathrm{~Hz}, 1 \mathrm{H}, 3-\mathrm{H}), 4.42$ (d, $J=7.6 \mathrm{~Hz}, 2 \mathrm{H}, 7-\mathrm{H})$, $4.51\left(\mathrm{~d}, J=11.8 \mathrm{~Hz}, 1 \mathrm{H}, \mathrm{CH}_{2}-\mathrm{Ph}\right), 4.57\left(\mathrm{~d}, J=11.8 \mathrm{~Hz}, 1 \mathrm{H}, \mathrm{CH}_{2}-\mathrm{Ph}\right), 4.59$ (d, $J=11.8 \mathrm{~Hz}, 1 \mathrm{H}$, 
$\left.\mathrm{CH}_{2} \mathrm{Ph}\right), 4.66$ (d, $\left.J=11.8 \mathrm{~Hz}, 1 \mathrm{H}, \mathrm{CH}_{2}-\mathrm{Ph}\right), 7.26-7.36$ (m, $10 \mathrm{H}$, arom. H); ${ }^{13} \mathrm{C}$ NMR (125 MHz, $\left.\mathrm{CD}_{2} \mathrm{Cl}_{2}\right): \delta 19.3\left(\mathrm{q}, \mathrm{SCH}_{3}\right), 20.4(\mathrm{~d}, \mathrm{C}-2), 21.4(\mathrm{~d}, \mathrm{C}-6), 56.1(\mathrm{~d}, \mathrm{C}-1), 63.7(\mathrm{t}, \mathrm{C}-5), 71.5,71.8(2 \mathrm{t}$, $\mathrm{CH}_{2}-\mathrm{Ph}$ ), 73.0 (d, C-3), 75.1 (t, C-7), 75.8 (d, C-4), 128.0, 128.1, 128.2, 128.4, 128.8, 128.9 (6d, Carom.), 138.7, 138.8 (2s, C-arom.), 216.6 (s, OCS); IR (film) $\tilde{v}=2913,2865,1453,1199,1047$, 963, 735, $696 \mathrm{~cm}^{-1}$; HRMS (ESI-Q-TOF): $\mathrm{m} / z$ calcd for $\mathrm{C}_{23} \mathrm{H}_{27} \mathrm{~S}_{2} \mathrm{O}_{4}[\mathrm{M}+\mathrm{H}]^{+}:$431.1351; found 431.1336 .

\section{General procedure for radical reactions}

The crude xanthate $9(1 \mathrm{mmol})$ was dissolved in $d r y$ benzene $(12 \mathrm{~mL})$ and tributyltin hydride $(0.4$ $\mathrm{mL}, 1.5 \mathrm{mmol})$ and AIBN (16 mg, $0.1 \mathrm{mmol})$ were added at room temperature under an argon atmosphere. Argon was bubbled through the solution for $30 \mathrm{~min}$, and then the mixture was heated by an oil bath $\left(100^{\circ} \mathrm{C}\right)$ under reflux for $2 \mathrm{~h}$. After this time another 0.1 equiv. of AIBN were added and heating was continued for $3 \mathrm{~h}$. The reaction mixture was allowed to cool to room temperature overnight. The residue obtained after removing of the solvent was taken up in acetonitrile $(50 \mathrm{~mL})$ and carefully washed with pentane $(5 \times 15 \mathrm{~mL})$. The combined pentane phases were re-extracted with acetonitrile $(1 \times 20 \mathrm{~mL})$. Removal of the solvent of the combined acetonitrile phases provided a pale yellow oil. The product ratios 5a:5b were directly determined by ${ }^{1} \mathrm{H}$ NMR (500 MHz). The crude products were purified by column chromatography with hexane / methyl-tert.butyl-ether (95:5) and afforded the deoxy sugars 5 in analytically pure form together with some mixture fractions.

\section{gluco:}

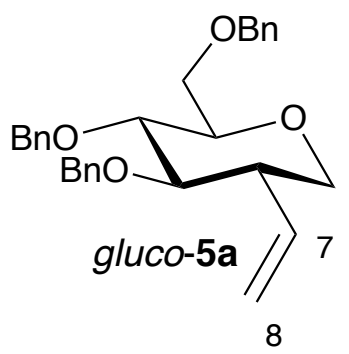

Main product gluco-5a: Colorless liquid (290 mg, 65\%). $R_{\mathrm{f}}=0.50$ (hexane/ethyl acetate 8:2); [a ${ }^{22}{ }_{\mathrm{D}}$ + 9.7 (c 1.03, $\left.\mathrm{CHCl}_{3}\right) ;{ }^{1} \mathrm{H}$ NMR $\left(500 \mathrm{MHz}, \mathrm{CD}_{2} \mathrm{Cl}_{2}\right.$ ): $\delta 2.60$ (ddddd, $J=11.6,10.3,8.3,4.9,0.9 \mathrm{~Hz}$, $1 \mathrm{H}, 2-\mathrm{H}), 3.27\left(\mathrm{t}, J=11.6 \mathrm{~Hz}, 1 \mathrm{H}, 1-\mathrm{H}_{\mathrm{ax}}\right), 3.40(\mathrm{ddd}, J=9.6,4.5,2.2 \mathrm{~Hz}, 1 \mathrm{H}, 5-\mathrm{H}), 3.43(\mathrm{dd}, J=$ 10.3, 8.9 Hz, $1 \mathrm{H}, 3-\mathrm{H}), 3.54$ (dd, $J=9.6,8.9 \mathrm{~Hz}, 1 \mathrm{H}, 4-\mathrm{H}), 3.67$ (dd, $J=10.5,4.5 \mathrm{~Hz}, 1 \mathrm{H}, 6-\mathrm{H})$, $3.71\left(\mathrm{dd}, J=10.5,2.2 \mathrm{~Hz}, 1 \mathrm{H}, 6^{\prime}-\mathrm{H}\right), 3.87$ (dd, $\left.J=11.6,4.9 \mathrm{~Hz}, 1 \mathrm{H}, 1-\mathrm{H}_{\text {equ. }}\right), 4.52$ (d, $J=10.8 \mathrm{~Hz}$, $\left.1 \mathrm{H}, \mathrm{CH}_{2}-\mathrm{Ph}\right), 4.54\left(\mathrm{~d}, J=12.2 \mathrm{~Hz}, 1 \mathrm{H}, \mathrm{CH}_{2}-\mathrm{Ph}\right), 4.61$ (d, J=12.2 Hz, $\left.1 \mathrm{H}, \mathrm{CH}_{2}-\mathrm{Ph}\right), 4.69$ (d, $J=$ $\left.10.7 \mathrm{~Hz}, 1 \mathrm{H}, \mathrm{CH}_{2}-\mathrm{Ph}\right), 4.75$ (d, $\left.J=10.7 \mathrm{~Hz}, 1 \mathrm{H}, \mathrm{CH}_{2}-\mathrm{Ph}\right), 4.82$ (d, J=10.8 Hz, $1 \mathrm{H}, \mathrm{CH}_{2}-\mathrm{Ph}$ ), 5.16 (dd, $J=10.3,1.4 \mathrm{~Hz}, 1 \mathrm{H}, 8-\mathrm{H}), 5.23$ (ddd, $J=17.3,1.4,0.9 \mathrm{~Hz}, 1 \mathrm{H}, 8$ '-H), 5.64 (ddd, $J=17.3$, 
10.3, 8.3 Hz, $1 \mathrm{H}, 7-\mathrm{H}), 7.15-7.35$ (m, $15 \mathrm{H}$, arom. H); ${ }^{13} \mathrm{C} \mathrm{NMR}\left(125 \mathrm{MHz}, \mathrm{CD}_{2} \mathrm{Cl}_{2}\right): \delta 48.4(\mathrm{~d}, \mathrm{C}-$ 2), 69.3 (t, C-6), 70.1 (t, C-1), 73.7, 74.9, 75.1 (3t, $\left.\mathrm{CH}_{2}-\mathrm{Ph}\right), 78.9$ (d, C-4), 79.8 (d, C-5), 85.0 (d, C3), 118.5 (t, C-8), 127.7, 127.8, 127.9, 128.0, 128.1, 128.2, 128.4, 128.5, 128.6 (9d, C-arom.), 135.0 (d, C-7), 138.1, 138.3, 138.5 (3s, C-arom.); IR (film) $\tilde{v}=3030,2855,1453,1088,735,696 \mathrm{~cm}^{-1}$; HRMS (ESI-Q-TOF): $m / z$ calcd for $\mathrm{C}_{29} \mathrm{H}_{33} \mathrm{O}_{4}[\mathrm{M}-\mathrm{H}]^{+}: 445.2379$ found 445.2381; Elemental analysis: calcd (\%) for $\mathrm{C}_{29} \mathrm{H}_{32} \mathrm{O}_{4}$ (444.57): C 78.35, H 7.25; found: C 78.18, H 7.23.

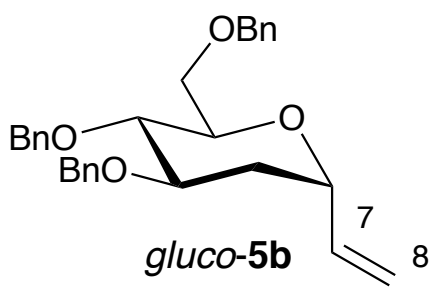

Side product gluco-5b: Colorless liquid (80 mg, 18\%). $R_{\mathrm{f}}=0.37$ (hexane/ethyl acetate 8:2); $[\mathrm{a}]^{22}{ }_{\mathrm{D}}+$ 46.8 (c 1.01, $\left.\mathrm{CHCl}_{3}\right) ;{ }^{1} \mathrm{H} \mathrm{NMR}\left(500 \mathrm{MHz}, \mathrm{CD}_{2} \mathrm{Cl}_{2}\right.$ ): $\delta 1.83$ (ddd, $J=13.3,10.0,5.4 \mathrm{~Hz}, 1 \mathrm{H}, 2-\mathrm{H}_{\text {ax. }}$ ), $2.20\left(\mathrm{ddd}, J=13.3,4.4,3.7 \mathrm{~Hz}, 1 \mathrm{H}, 2-\mathrm{H}_{\text {equ. }}\right), 3.51$ (dd, $\left.J=7.9,7.7 \mathrm{~Hz}, 1 \mathrm{H}, 4-\mathrm{H}\right), 3.67-3.77$ (m, 3 H, 5-H, 6-H), 3.78 (ddd, $J=10.0,7.7,4.4$ Hz, 1 H, 3-H), 4.50-4.57 (m, 1 H, 1-H), 4.52 (d, $J=12.0$ $\left.\mathrm{Hz}, 1 \mathrm{H}, \mathrm{CH}_{2}-\mathrm{Ph}\right), 4.56\left(\mathrm{~d}, J=12.0 \mathrm{~Hz}, 1 \mathrm{H}, \mathrm{CH}_{2}-\mathrm{Ph}\right), 4.58\left(\mathrm{~d}, J=11.7 \mathrm{~Hz}, 1 \mathrm{H}, \mathrm{CH}_{2}-\mathrm{Ph}\right), 4.60(\mathrm{~d}, J$ $\left.=11.1 \mathrm{~Hz}, 1 \mathrm{H}, \mathrm{CH}_{2}-\mathrm{Ph}\right), 4.66\left(\mathrm{~d}, J=11.7 \mathrm{~Hz}, 1 \mathrm{H}, \mathrm{CH}_{2}-\mathrm{Ph}\right), 4.83\left(\mathrm{~d}, J=11.1 \mathrm{~Hz}, 1 \mathrm{H}, \mathrm{CH}_{2}-\mathrm{Ph}\right)$, $5.21(\mathrm{dd}, J=17.1,2.0,1.4 \mathrm{~Hz}, 1 \mathrm{H}, 8-\mathrm{H}), 5.24\left(\mathrm{dd}, J=11.3,2.0 \mathrm{~Hz}, 1 \mathrm{H}, 8^{\prime}-\mathrm{H}\right), 5.91$ (ddd, $J=17.1$, 11.3, 4.2 Hz, $1 \mathrm{H}, 7-\mathrm{H}), 7.26-7.34\left(\mathrm{~m}, 15 \mathrm{H}\right.$, arom. H); ${ }^{13} \mathrm{C} \mathrm{NMR}\left(125 \mathrm{MHz}, \mathrm{CD}_{2} \mathrm{Cl}_{2}\right): \delta 33.2(\mathrm{t}, \mathrm{C}-$ 2), 70.0 (t, C-6), 71.9 (d, C-1), 71.8, 73.7, 74.6 (3t, $\left.\mathrm{CH}_{2}-\mathrm{Ph}\right), 73.7$ (d, C-5), 77.2 (d, C-3), 78.2 (d, C4), 116.8 (t, C-8), 127.7, 127.8, 127.9, 128.1, 128.2, 128.3, 128.6, 128.7, 128.8 (9d, C-arom.), 138.1 (d, C-7), 139.0, 139.2, 139.3 (3s, C-arom.); IR (film) $\tilde{v}=3030,2863,1453,1093,1027,735,696$ $\mathrm{cm}^{-1}$; HRMS (ESI-Q-TOF): $\mathrm{m} / z$ calcd for $\mathrm{C}_{29} \mathrm{H}_{33} \mathrm{O}_{4}[\mathrm{M}-\mathrm{H}]^{+}: 445.2379$ found 445.2380; Elemental analysis: calcd (\%) for $\mathrm{C}_{29} \mathrm{H}_{32} \mathrm{O}_{4}$ (444.57): C 78.35, H 7.25; found: C 78.12, H 7.32.

\section{manno:}

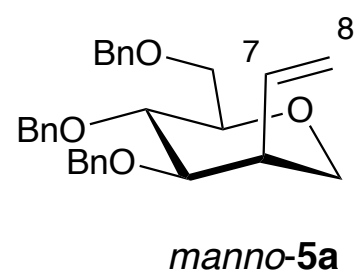

Main product manno-5a:: Colorless liquid (245 mg, 55\%). $R_{\mathrm{f}}=0.53$ (hexane/ethyl acetate 8:2); [a] ${ }^{22}$ - 14.2 (c 1.03, $\mathrm{CHCl}_{3}$ ); ${ }^{1} \mathrm{H}$ NMR $\left(500 \mathrm{MHz}, \mathrm{CD}_{2} \mathrm{Cl}_{2}\right.$ ): $\delta 2.84$ (dddddd, $J=8.6,5.3,2.4,1.9,1.0,0.6$ $\mathrm{Hz}, 1 \mathrm{H}, 2-\mathrm{H}), 3.38$ (ddd, $J=9.3,4.5,2.7 \mathrm{~Hz}, 1 \mathrm{H}, 5-\mathrm{H}), 3.58$ (dd, $J=11.7,2.4 \mathrm{~Hz}, 1 \mathrm{H}, 1-\mathrm{H}_{\text {ax. }}$ ), 3.63 (dd, $J=9.3,9.0 \mathrm{~Hz}, 1 \mathrm{H}, 4-\mathrm{H}), 3.69$ (dd, $J=10.7,4.5 \mathrm{~Hz}, 1 \mathrm{H}, 6-\mathrm{H}), 3.70$ (dd, $J=10.7,2.7 \mathrm{~Hz}, 1 \mathrm{H}$, 6'-H), $3.72(\mathrm{dd}, J=9.0,5.3 \mathrm{~Hz}, 1 \mathrm{H}, 3-\mathrm{H}), 3.93$ (dd, $\left.J=11.7,1.9 \mathrm{~Hz}, 1 \mathrm{H}, 1-\mathrm{H}_{\text {equ. }}\right), 4.49$ (d, $J=11.9$ 
$\left.\mathrm{Hz}, 1 \mathrm{H}, \mathrm{CH}_{2}-\mathrm{Ph}\right), 4.51$ (d, $\left.J=10.9 \mathrm{~Hz}, 1 \mathrm{H}, \mathrm{CH}_{2}-\mathrm{Ph}\right), 4.52$ (d, $\left.J=11.4 \mathrm{~Hz}, 1 \mathrm{H}, \mathrm{CH}_{2}-\mathrm{Ph}\right), 4.57$ (d, $J$ $\left.=11.9 \mathrm{~Hz}, 1 \mathrm{H}, \mathrm{CH}_{2}-\mathrm{Ph}\right), 4.71\left(\mathrm{~d}, J=11.4 \mathrm{~Hz}, 1 \mathrm{H}, \mathrm{CH}_{2}-\mathrm{Ph}\right), 4.86\left(\mathrm{~d}, J=10.9 \mathrm{~Hz}, 1 \mathrm{H}, \mathrm{CH}{ }_{2}-\mathrm{Ph}\right)$, 5.27 (ddd, $J=10.4,1.9,0.6 \mathrm{~Hz}, 1 \mathrm{H}, 8-\mathrm{H}), 5.27$ (ddd, $\left.J=17.3,1.9,1.0 \mathrm{~Hz}, 1 \mathrm{H}, 8^{\prime}-\mathrm{H}\right), 6.10$ (ddd, $J=$ 17.3, 10.4, 8.6 Hz, $1 \mathrm{H}, 7-\mathrm{H}), 7.20-7.38$ (m, $15 \mathrm{H}$, arom. H); ${ }^{13} \mathrm{C}$ NMR (125 MHz, $\left.\mathrm{CD}_{2} \mathrm{Cl}_{2}\right): \delta 43.7$ (d, C-2), 70.1 (t, C-1), 70.2 (t, C-6), 70.9, 73.7, 75.3 (3t, $\left.\mathrm{CH}_{2}-\mathrm{Ph}\right), 75.5$ (d, C-4), 80.4 (d, C-5), 83.0 (d, C-3), 117.7 (t, C-8), 127.8, 127.9, 128.0, 128.1, 128.2, 128.3, 128.5, 128.6, 128.7 (9d, C-arom.), 136.3 (d, C-7), 138.9, 139.0, 139.2 (3s, C-arom.); IR (film) $\tilde{v}=3063,3030,2918,2854,1453,1093$, 1024, 916, 734, $696 \mathrm{~cm}^{-1}$; HRMS (ESI-Q-TOF): $\mathrm{m} / z$ calcd for $\mathrm{C}_{29} \mathrm{H}_{33} \mathrm{O}_{4}[\mathrm{M}-\mathrm{H}]^{+}: 445.2379$ found 445.2373; Elemental analysis: calcd (\%) for $\mathrm{C}_{29} \mathrm{H}_{32} \mathrm{O}_{4}$ (444.57): C 78.35, H 7.25; found: C 78.56, H 7.29 .

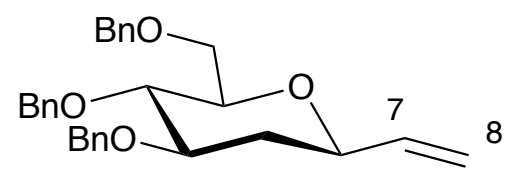

manno-5b

Side product manno-5b: Colorless liquid (100 mg, 22\%). $R_{\mathrm{f}}=0.62$ (hexane/ethyl acetate 8:2); $[\mathrm{a}]^{22}{ }_{\mathrm{D}}$ - $5.5\left(\mathrm{c} 1.00, \mathrm{CHCl}_{3}\right) ;{ }^{1} \mathrm{H}$ NMR $\left(500 \mathrm{MHz}, \mathrm{CD}_{2} \mathrm{Cl}_{2}\right): \delta 1.48$ (ddd, $J=12.8,11.5,11.3 \mathrm{~Hz}, 1 \mathrm{H}, 2-$ $\mathrm{H}_{\text {ax. }}$ ), 2.23 (ddd, $J=12.8,5.0,2.0 \mathrm{~Hz}, 1 \mathrm{H}, 2-\mathrm{H}_{\text {equ. }}$ ), 3.42 (ddd, $\left.J=9.7,3.9,2.2 \mathrm{~Hz}, 1 \mathrm{H}, 5-\mathrm{H}\right), 3.46$ (dd, $J=9.7,8.2 \mathrm{~Hz}, 1 \mathrm{H}, 4-\mathrm{H}), 3.68$ (ddd, $J=11.3,8.2,5.0 \mathrm{~Hz}, 1 \mathrm{H}, 3-\mathrm{H}), 3.71$ (dd, $J=10.8,3.9 \mathrm{~Hz}$, $1 \mathrm{H}, 6-\mathrm{H}), 3.74$ (dd, $J=10.8,2.2 \mathrm{~Hz}, 1 \mathrm{H}, 6$ '-H), 3.89 (ddt, $J=11.5,5.7,2.0,1.5 \mathrm{~Hz}, 1 \mathrm{H}, 1-\mathrm{H}), 4.52$ $\left(\mathrm{d}, J=11.9 \mathrm{~Hz}, 1 \mathrm{H}, \mathrm{CH}_{2}-\mathrm{Ph}\right), 4.57\left(\mathrm{~d}, J=10.9 \mathrm{~Hz}, 1 \mathrm{H}, \mathrm{CH}_{2}-\mathrm{Ph}\right), 4.58\left(\mathrm{~d}, J=11.9 \mathrm{~Hz}, 1 \mathrm{H}, \mathrm{CH}_{2^{-}}\right.$ $\mathrm{Ph}), 4.61\left(\mathrm{~d}, J=11.7 \mathrm{~Hz}, 1 \mathrm{H}, \mathrm{CH}_{2}-\mathrm{Ph}\right), 4.71\left(\mathrm{~d}, J=11.7 \mathrm{~Hz}, 1 \mathrm{H}, \mathrm{CH}_{2}-\mathrm{Ph}\right), 4.91$ (d, $J=10.9 \mathrm{~Hz}, 1$ $\left.\mathrm{H}, \mathrm{CH}_{2} \mathrm{Ph}\right), 5.14$ (dt, $\left.J=10.6,1.5 \mathrm{~Hz}, 1 \mathrm{H}, 8-\mathrm{H}\right), 5.28$ (dt, $\left.J=17.3,1.5 \mathrm{~Hz}, 1 \mathrm{H}, 8^{\prime}-\mathrm{H}\right), 5.89$ (ddd, $J=$ 17.3, 10.6, $5.7 \mathrm{~Hz}, 1 \mathrm{H}, 7-\mathrm{H}), 7.22-7.38\left(\mathrm{~m}, 15 \mathrm{H}\right.$, arom. H); ${ }^{13} \mathrm{C} \mathrm{NMR}\left(125 \mathrm{MHz}, \mathrm{CD}_{2} \mathrm{Cl}_{2}\right): \delta 37.1(\mathrm{t}$, C-2), 70.1 (t, C-6), 71.5, 73.7, 76.5 (3t, $\left.\mathrm{CH}_{2}-\mathrm{Ph}\right), 76.5$ (d, C-1), 78.7 (d, C-4), 79.2 (d, C-5), 81.3 (d, C-3), 115.5 (t, C-8), 127.8, 127.9, 128.0, 128.1, 128.2, 128.4, 128.5, 128.6, 128.7 (9d, C-arom.), 138.4 (d, C-7), 138.5, 138.9, 139.3 (3s, C-arom.); IR (film) $\tilde{v}=3065,3029,2920,2856,1453,1361$, 1093, 1024, 917, 734, $696 \mathrm{~cm}^{-1}$; HRMS (ESI-Q-TOF): $\mathrm{m} / z$ calcd for $\mathrm{C}_{29} \mathrm{H}_{33} \mathrm{O}_{4}[\mathrm{M}-\mathrm{H}]^{+}: 445.2379$ found 445.2370 . 


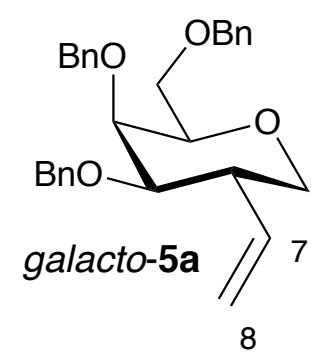

Sole radical product galacto-5a: Colorless liquid (350 mg, 79\%). $R_{\mathrm{f}}=0.39$ (hexane/ethyl acetate 8:2); $[\mathrm{a}]^{22}{ }_{\mathrm{D}}+23.8\left(\mathrm{c} 1.03, \mathrm{CHCl}_{3}\right) ;{ }^{1} \mathrm{H}$ NMR $\left(600 \mathrm{MHz}, \mathrm{CD}_{2} \mathrm{Cl}_{2}\right): \delta 2.89$ (dddddd, $J=11.3,10.7$, 8.0, 5.0, 1.1, $0.8 \mathrm{~Hz}, 1 \mathrm{H}, 2-\mathrm{H}), 3.22$ (dd, $\left.J=11.5,11.3 \mathrm{~Hz}, 1 \mathrm{H}, 1-\mathrm{H}_{\mathrm{ax} .}\right), 3.38$ (dd, $J=10.7,2.6 \mathrm{~Hz}, 1$ H, 3-H), 3.50 (ddd, $J=6.4,6.1,1.0 \mathrm{~Hz}, 1 \mathrm{H}, 5-\mathrm{H}), 3.56(\mathrm{dd}, J=9.3,6.1 \mathrm{~Hz}, 1 \mathrm{H}, 6-\mathrm{H}), 3.58(\mathrm{dd}, J=$ 9.3, 6.4 Hz, $1 \mathrm{H}, 6$ '-H), 3.84 (dd, $J=11.5,5-0 \mathrm{~Hz}, 1 \mathrm{H}, 1-\mathrm{H}_{\text {equ. }}$ ), 3.95 (dd, $\left.J=2.6,1.0 \mathrm{~Hz}, 1 \mathrm{H}, 4-\mathrm{H}\right)$, $4.45\left(\mathrm{~d}, J=11.8 \mathrm{~Hz}, 1 \mathrm{H}, \mathrm{CH}_{2}-\mathrm{Ph}\right), 4.51\left(\mathrm{~d}, J=11.8 \mathrm{~Hz}, 1 \mathrm{H}, \mathrm{CH}_{2}-\mathrm{Ph}\right), 4.53(\mathrm{~d}, J=11.7 \mathrm{~Hz}, 1 \mathrm{H}$, $\mathrm{CH}_{2} \mathrm{Ph}$ ), 4.54 (d, $\left.J=11.0 \mathrm{~Hz}, 1 \mathrm{H}, \mathrm{CH}_{2}-\mathrm{Ph}\right), 4.72$ (d, $\left.J=11.7 \mathrm{~Hz}, 1 \mathrm{H}, \mathrm{CH}_{2}-\mathrm{Ph}\right), 4.89$ (d, $J=11.0$ $\mathrm{Hz}, 1 \mathrm{H}, \mathrm{CH}_{2}-\mathrm{Ph}$ ), 5.15 (ddd, $\left.J=10.5,1.7,0.8 \mathrm{~Hz}, 1 \mathrm{H}, 8-\mathrm{H}\right), 5.18$ (ddd, $J=17.4,1.7,1.1 \mathrm{~Hz}, 1 \mathrm{H}$, 8'-H), 5.65 (ddd, $J=17.4,10.5,8.0 \mathrm{~Hz}, 1 \mathrm{H}, 7-\mathrm{H}), 7.29-7.36$ (m, $15 \mathrm{H}$, arom. H); ${ }^{13} \mathrm{C}$ NMR (150 $\mathrm{MHz}, \mathrm{CD}_{2} \mathrm{Cl}_{2}$ ): $\delta 42.6$ (d, C-2), 70.1 (t, C-6), 70.7 (t, C-1), 71.8, 73.8, 75.0 (3t, $\left.\mathrm{CH}_{2}-\mathrm{Ph}\right), 72.9$ (d, C4), 78.3 (d, C-5), 82.6 (d, C-3), 117.8 (t, C-8), 127.8, 127.9, 128.0, 128.1, 128.2, 128.3, 128.5, 128.6, 128.7 (9d, C-arom.), 1356.5 (d, C-7), 138.7, 138.8, 139.3 (3s, C-arom.); IR (film) $\tilde{v}=3029,2860$, 1454, 1354, 1084, 864, 735, $696 \mathrm{~cm}^{-1}$; HRMS (ESI-Q-TOF): $\mathrm{m} / z$ calcd for $\mathrm{C}_{29} \mathrm{H}_{33} \mathrm{O}_{4}[\mathrm{M}-\mathrm{H}]^{+}$: 445.2379 found 445.2372 .

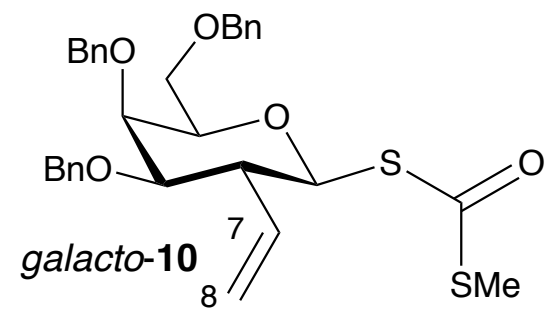

Thermolysis side product galacto-10: Colorless liquid ( $80 \mathrm{mg}, 14 \%) . R_{\mathrm{f}}=0.41$ (hexane/ethyl acetate 8:2); $[\mathrm{a}]^{22}{ }_{\mathrm{D}}+14.4\left(\mathrm{c} 1.02, \mathrm{CHCl}_{3}\right) ;{ }^{1} \mathrm{H} \mathrm{NMR}\left(600 \mathrm{MHz}, \mathrm{CD}_{2} \mathrm{Cl}_{2}\right): \delta 2.42$ (s, $\left.3 \mathrm{H}, \mathrm{SMe}\right), 2.86(\mathrm{dtd}, J$ $=11.1,9.4,0.6 \mathrm{~Hz}, 1 \mathrm{H}, 2-\mathrm{H}), 3.46(\mathrm{dd}, J=9.4,2.6 \mathrm{~Hz}, 1 \mathrm{H}, 3-\mathrm{H}), 3.56(\mathrm{dd}, J=9.4,6.0 \mathrm{~Hz}, 1 \mathrm{H}, 6-$ H), $3.58(\mathrm{dd} J=9.4,6.7 \mathrm{~Hz}, 1 \mathrm{H}, 6$ '-H), 3.72 (ddd, $J=6.7,6.0,1.0 \mathrm{~Hz}, 1 \mathrm{H}, 5-\mathrm{H}), 3.95$ (dd, $J=2.6$, $1.0 \mathrm{~Hz}, 1 \mathrm{H}, 4-\mathrm{H}), 4.44$ (d, $\left.J=11.7 \mathrm{~Hz}, 1 \mathrm{H}, \mathrm{CH}_{2}-\mathrm{Ph}\right), 4.50$ (d, $\left.J=11.7 \mathrm{~Hz}, 1 \mathrm{H}, \mathrm{CH}_{2}-\mathrm{Ph}\right), 4.53$ (d, $J$ $\left.=11.5 \mathrm{~Hz}, 1 \mathrm{H}, \mathrm{CH}_{2}-\mathrm{Ph}\right), 4.55\left(\mathrm{~d}, J=11.2 \mathrm{~Hz}, 1 \mathrm{H}, \mathrm{CH}_{2}-\mathrm{Ph}\right), 4.67\left(\mathrm{~d}, J=11.5 \mathrm{~Hz}, 1 \mathrm{H}, \mathrm{CH}_{2}-\mathrm{Ph}\right)$, $4.87\left(\mathrm{~d}, J=11.2 \mathrm{~Hz}, 1 \mathrm{H}, \mathrm{CH}_{2} \mathrm{Ph}\right), 5.23(\mathrm{~d}, J=11.1 \mathrm{~Hz}, 1 \mathrm{H}, 1-\mathrm{H}), 5.24$ (dd, $J=10.3,1.6 \mathrm{~Hz}, 1 \mathrm{H}$, 8-H), 5.26 (ddd, $J=17.1,1.6,0.6 \mathrm{~Hz}, 1 \mathrm{H}, 8$ '-H), 5.53 (ddd, $J=17.1,10.3,9.2 \mathrm{~Hz}, 1 \mathrm{H}, 7-\mathrm{H}), 7.30$ 7.39 (m, $15 \mathrm{H}$, arom. H); ${ }^{13} \mathrm{C}$ NMR (125 MHz, $\left.\mathrm{CD}_{2} \mathrm{Cl}_{2}\right): \delta 13.4$ (q, SMe), 47.0 (d, C-2), $69.2(\mathrm{t}, \mathrm{C}-$ 6), 71.9 (d, C-4), 72.3, 73.7, 75.1 (3t, $\left.\mathrm{CH}_{2}-\mathrm{Ph}\right), 78.2$ (d, C-5), 81.9 (d, C-3), 83.0 (d, C-1), 120.5 (t, 
C-8), 127.9, 128.0, 128.2, 128.3, 128.4, 128.5, 128.6, 128.7, 128.8 (9d, C-arom.), 134.8 (d, C-7), 138.4, 138.5, 139.1 (3s, C-arom.), 188.1 (s, COSMe); IR (film) $\tilde{v}=3027,2866,1647,1099,863$, 736, 698, $669 \mathrm{~cm}^{-1}$; HRMS (ESI-Q-TOF): $\mathrm{m} / z$ calcd for $\mathrm{C}_{31} \mathrm{H}_{35} \mathrm{O}_{5} \mathrm{~S}_{2}[\mathrm{M}-\mathrm{H}]^{+}:$551.1926; found 551.1931 .

\section{arabino:}

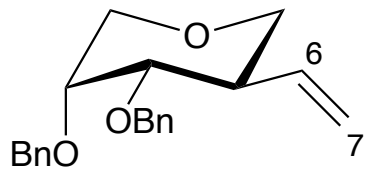

arabino-5a

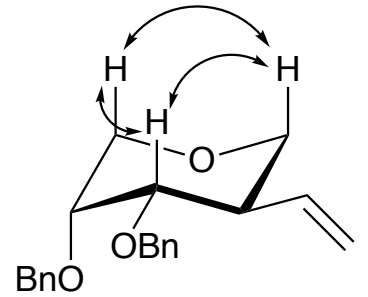

arabino-5a

Main product arabino-5a: Colorless liquid (260 mg, 80\%). $R_{\mathrm{f}}=0.33$ (hexane/ethyl acetate 8:2); $[\mathrm{a}]_{\mathrm{D}}^{25}-58.2\left(\mathrm{c} 1.03, \mathrm{CHCl}_{3}\right) ;{ }^{1} \mathrm{H}$ NMR $\left(600 \mathrm{MHz}, \mathrm{CD}_{2} \mathrm{Cl}_{2}\right): \delta 2.84$ (dddddd, $J=9.4,7.7,7.3,4.5$, 1.2, $0.9 \mathrm{~Hz}, 1 \mathrm{H}, 2-\mathrm{H}), 3.24$ (dd, $J=11.4,9.4 \mathrm{~Hz}, 1 \mathrm{H}, 1-\mathrm{H}_{\text {ax. }}$ ), 3.37 (dd, $\left.J=12.2,1.7 \mathrm{~Hz}, 1 \mathrm{H}, 5-\mathrm{H}\right)$, $3.43(\mathrm{dd}, J=7.4,2.9 \mathrm{~Hz}, 1 \mathrm{H}, 3-\mathrm{H}), 3.73-3.75(\mathrm{~m}, 1 \mathrm{H}, 4-\mathrm{H}), 3.81$ (dd, $J=11.4,4.5 \mathrm{~Hz}, 1 \mathrm{H}, 1$ $\mathrm{H}_{\text {equ. }}$ ), 4.03 (dd, $\left.J=12.2,3.9 \mathrm{~Hz}, 1 \mathrm{H}, 5^{\prime}-\mathrm{H}\right), 4.48$ (d, $\left.J=11.7 \mathrm{~Hz}, 1 \mathrm{H}, \mathrm{CH}_{2}-\mathrm{Ph}\right), 4.61$ (d, $J=11.7$ $\left.\mathrm{Hz}, 1 \mathrm{H}, \mathrm{CH}_{2}-\mathrm{Ph}\right), 4.61\left(\mathrm{~d}, J=11.9 \mathrm{~Hz}, 1 \mathrm{H}, \mathrm{CH}_{2}-\mathrm{Ph}\right), 4.67$ (d, $\left.J=11.9 \mathrm{~Hz}, 1 \mathrm{H}, \mathrm{CH}_{2}-\mathrm{Ph}\right), 5.15$ (ddd, $J=10.5,1.7,0.9 \mathrm{~Hz}, 1 \mathrm{H}, 7-\mathrm{H}), 5.18$ (ddd, $\left.J=17.4,1.7,1.2 \mathrm{~Hz}, 1 \mathrm{H}, 7^{\prime}-\mathrm{H}\right), 5.72$ (ddd, $J=$ 17.4, 10.5, 7.7 Hz, $1 \mathrm{H}, 6-\mathrm{H}), 7.29-7.40$ (m, $10 \mathrm{H}$, arom. H); ${ }^{13} \mathrm{C} \mathrm{NMR}\left(125 \mathrm{MHz}, \mathrm{CD}_{2} \mathrm{Cl}_{2}\right): \delta 42.9$ (d, C-2), 67.8 (t, C-5), 70.1 (t, C-1), $71.2,71.6\left(2 \mathrm{t}, \mathrm{CH}_{2}-\mathrm{Ph}\right), 72.3$ (d, C-4), 80.0 (d, C-3), 117.5 (t, C7), 127.8, 127.9, 128.0, 128.2, 128.6, 128.7 (6d, C-arom.), 136.9 (d, C-6), 139.0, 139.1 (2s, Carom.); IR (film) $\tilde{v}=3029,2959,2863,1454,1351,1100,1060,733,696 \mathrm{~cm}^{-1}$; HRMS (ESI-QTOF): $m / z$ calcd for $\mathrm{C}_{21} \mathrm{H}_{25} \mathrm{O}_{3}[\mathrm{M}-\mathrm{H}]^{+}: 325.1804$ found 325.1787 ; Elemental analysis: calcd (\%) for $\mathrm{C}_{21} \mathrm{H}_{24} \mathrm{O}_{3}$ (324.42): C 77.75, H 7.46; found: C 77.63, H 7.31.

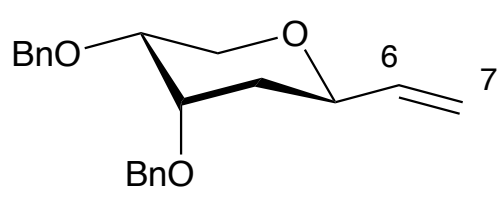

arabino-5b

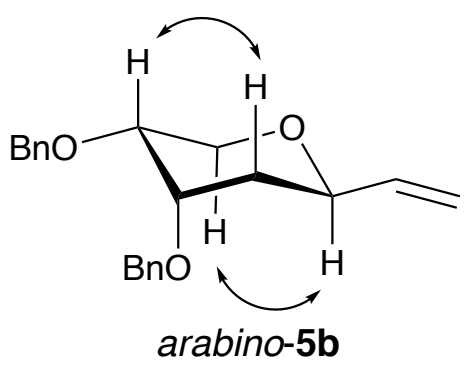

arabino-5b

Side product arabino-5b: Colorless liquid (15 mg, 5\%). $R_{\mathrm{f}}=0.41$ (hexane/ethyl acetate 8:2); $[\mathrm{a}]^{25}{ }_{\mathrm{D}}^{-}$ $47.7\left(\mathrm{c} 0.61, \mathrm{CHCl}_{3}\right) ;{ }^{1} \mathrm{H} \mathrm{NMR}\left(500 \mathrm{MHz}, \mathrm{CD}_{2} \mathrm{Cl}_{2}\right.$ ): $\delta 1.47$ (ddd, $J=14.0,11.3,2.4 \mathrm{~Hz}, 1 \mathrm{H}, 2-$ $\left.\mathrm{H}_{\text {ax. }}\right), 2.03$ (ddd, $\left.J=14.0,4.1,2.4 \mathrm{~Hz}, 1 \mathrm{H}, 2-\mathrm{H}_{\text {equ. }}\right), 3.55$ (ddd, $\left.J=9.3,6.2,2.7 \mathrm{~Hz}, 1 \mathrm{H}, 4-\mathrm{H}\right), 3.80-$ $3.84(\mathrm{~m}, 2 \mathrm{H}, 5-\mathrm{H}), 4.02$ (ddd, $J=4.1,2.7,2.4 \mathrm{~Hz}, 1 \mathrm{H}, 3-\mathrm{H}), 4.21$ (dddt, $J=11.3,5.5,2.4,1.6 \mathrm{~Hz}, 1$ 
$\mathrm{H}, 1-\mathrm{H}), 4.54\left(\mathrm{~d}, J=12.0 \mathrm{~Hz}, 1 \mathrm{H}, \mathrm{CH}_{2}-\mathrm{Ph}\right), 4.59$ (d, $\left.J=12.0 \mathrm{~Hz}, 1 \mathrm{H}, \mathrm{CH}_{2}-\mathrm{Ph}\right), 4.68$ (d, $J=12.0$ $\left.\mathrm{Hz}, 1 \mathrm{H}, \mathrm{CH}_{2}-\mathrm{Ph}\right), 4.72\left(\mathrm{~d}, J=12.0 \mathrm{~Hz}, 1 \mathrm{H}, \mathrm{CH}_{2}-\mathrm{Ph}\right), 5.07$ (dt, $\left.J=10.6,1.6 \mathrm{~Hz}, 1 \mathrm{H}, 7-\mathrm{H}\right), 5.21$ (dt, $\left.J=17.3,1.6 \mathrm{~Hz}, 1 \mathrm{H}, 7^{\prime}-\mathrm{H}\right), 5.80$ (ddd, $\left.J=17.3,10.6,5.5 \mathrm{~Hz}, 1 \mathrm{H}, 6-\mathrm{H}\right), 7.26-7.42$ (m, $10 \mathrm{H}$, arom. $\mathrm{H}) ;{ }^{13} \mathrm{C}$ NMR $\left(125 \mathrm{MHz}, \mathrm{CD}_{2} \mathrm{Cl}_{2}\right): \delta 36.0(\mathrm{t}, \mathrm{C}-2), 65.0(\mathrm{t}, \mathrm{C}-5), 71.3,72.2\left(2 \mathrm{t}, \mathrm{CH}_{2}-\mathrm{Ph}\right), 72.7(\mathrm{~d}, \mathrm{C}-$ 1), 72.7 (d, C-3), 76.2 (d, C-4), 115.0 (t, C-7), 127.8, 127.9, 128.0, 128.2, 128.7, 128.8 (6d, Carom.), 139.1 (d, C-6), 139.2, 139.7 (2s, C-arom.); IR (film) $\tilde{v}=3023,2960,2920,2854,1455$, 1259, 1082, 1021, 799, $697 \mathrm{~cm}^{-1}$; HRMS (ESI-Q-TOF): $m / z$ calcd for $\mathrm{C}_{21} \mathrm{H}_{25} \mathrm{O}_{3}[\mathrm{M}-\mathrm{H}]^{+}: 325.1804$ found 325.1800; Elemental analysis: calcd (\%) for $\mathrm{C}_{21} \mathrm{H}_{24} \mathrm{O}_{3}$ (324.42): C 77.75, $\mathrm{H}$ 7.46; found: C $77.58, \mathrm{H} 7.66$.

xylo:
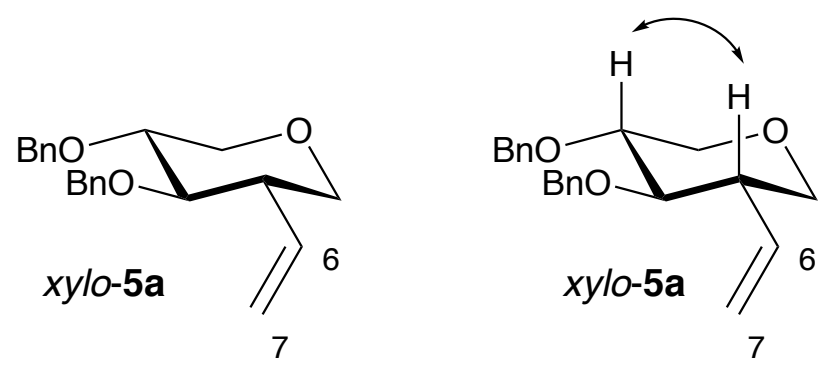

Main product $x y l o-5 a:$ Colorless liquid (185 mg, 57\%). $R_{\mathrm{f}}=0.44$ (hexane/ethyl acetate 8:2); $[\mathrm{a}]^{21}{ }_{\mathrm{D}}^{-}$ 5.1 (c 1.01, $\left.\mathrm{CHCl}_{3}\right) ;{ }^{1} \mathrm{H}$ NMR $\left(500 \mathrm{MHz}, \mathrm{CD}_{2} \mathrm{Cl}_{2}\right.$ ): $\delta 2.45$ (dddddd, $J=10.9,9.9,8.0,4.9,1.0,0.7$ $\mathrm{Hz}, 1 \mathrm{H}, 2-\mathrm{H}), 3.16\left(\mathrm{dd}, J=11.2,10.2 \mathrm{~Hz}, 1 \mathrm{H}, 5-\mathrm{H}_{\mathrm{ax}}\right), 3.20\left(\mathrm{dd}, J=11.5,10.9 \mathrm{~Hz}, 1 \mathrm{H}, 1-\mathrm{H}_{\mathrm{ax}}\right)$, $3.35(\mathrm{dd}, J=9.9,8.4 \mathrm{~Hz}, 1 \mathrm{H}, 3-\mathrm{H}), 3.54$ (ddd, $J=10.2,8.4,5.1 \mathrm{~Hz}, 1 \mathrm{H}, 4-\mathrm{H}), 3.76$ (ddd, $J=11.5$, 4.9, $\left.1.3 \mathrm{~Hz}, 1 \mathrm{H}, 1-\mathrm{H}_{\text {equ. }}\right), 4.03$ (ddd, $J=11.2,5.1,1.3 \mathrm{~Hz}, 1 \mathrm{H}, 5-\mathrm{H}_{\text {equ. }}$ ), 4.64 (d, $J=11.6 \mathrm{~Hz}, 1 \mathrm{H}$, $\left.\mathrm{CH}_{2}-\mathrm{Ph}\right), 4.66\left(\mathrm{~d}, J=11.1 \mathrm{~Hz}, 1 \mathrm{H}, \mathrm{CH}_{2}-\mathrm{Ph}\right), 4.69$ (d, $\left.J=11.6 \mathrm{~Hz}, 1 \mathrm{H}, \mathrm{CH}_{2}-\mathrm{Ph}\right), 4.82$ (d, $J=11.1$ $\mathrm{Hz}, 1 \mathrm{H}, \mathrm{CH}_{2}-\mathrm{Ph}$ ), 5.15 (ddd, $\left.J=10.4,1.7,0.7 \mathrm{~Hz}, 1 \mathrm{H}, 7-\mathrm{H}\right), 5.19$ (ddd, $J=17.3,1.7,1.0 \mathrm{~Hz}, 1 \mathrm{H}$, 7'-H), 5.71 (ddd, $J=17.3,10.4,8.0 \mathrm{~Hz}, 1 \mathrm{H}, 6-\mathrm{H}), 7.26-7.34$ (m, $10 \mathrm{H}$, arom. H); ${ }^{13} \mathrm{C}$ NMR $(125$ $\mathrm{MHz}, \mathrm{CD}_{2} \mathrm{Cl}_{2}$ ): $\delta 47.9$ (d, C-2), 69.4 (t, C-5), 70.9 (t, C-1), 73.2, 74.9 (2t, $\left.\mathrm{CH}_{2}-\mathrm{Ph}\right), 79.4$ (d, C-4), 83.7 (d, C-3), 117.9 (t, C-7), 127.9, 128.0, 128.2, 128.3, 128.6, 128.8 (6d, C-arom.), 136.0 (d, C-6), 139.2, 139.6 (2s, C-arom.); IR (film) $\tilde{v}=3030,2957,2847,1454,1356,1206,1086,916,736,696$ $\mathrm{cm}^{-1}$; HRMS (ESI-Q-TOF): $\mathrm{m} / z$ calcd for $\mathrm{C}_{21} \mathrm{H}_{25} \mathrm{O}_{3}[\mathrm{M}-\mathrm{H}]^{+}: 325.1804$ found 325.1788 ; Elemental analysis: calcd (\%) for $\mathrm{C}_{21} \mathrm{H}_{24} \mathrm{O}_{3}$ (324.42): C 77.75, H 7.46; found: C 77.99, $\mathrm{H}$ 7.61.

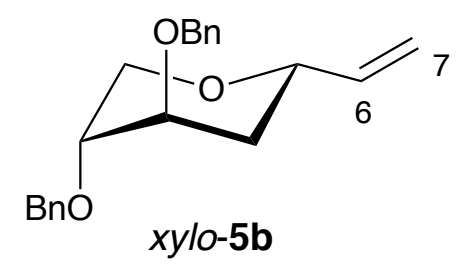

Side product xylo-5b: Colorless liquid (52 mg, 16\%). $R_{\mathrm{f}}=0.44$ (hexane/ethyl acetate 8:2); [a] ${ }^{21}{ }_{\mathrm{D}}^{-}$ 48.6 (c 0.59, $\left.\mathrm{CHCl}_{3}\right)$; ${ }^{1} \mathrm{H} \mathrm{NMR}\left(500 \mathrm{MHz}, \mathrm{CD}_{2} \mathrm{Cl}_{2}\right.$ ): $\delta 1.79$ (ddd, $J=14.0,3.8,3.4 \mathrm{~Hz}, 1 \mathrm{H}, 2-\mathrm{H}_{\text {equ. }}$ ), 
$1.86\left(\mathrm{ddd}, J=14.0,10.8,3.0 \mathrm{~Hz}, 1 \mathrm{H}, 2-\mathrm{H}_{\mathrm{ax}}\right), 3.20$ (dd, $\left.J=11.5,10.9 \mathrm{~Hz}, 1 \mathrm{H}, 1-\mathrm{H}_{\mathrm{ax}}\right), 3.36$ (ddd, $J$ $=3.8,3.0,1.8 \mathrm{~Hz}, 1 \mathrm{H}, 4-\mathrm{H}), 3.78(\mathrm{ddt}, J=3.8,3.0,1.8 \mathrm{~Hz}, 1 \mathrm{H}, 3-\mathrm{H}), 3.16(\mathrm{dd}, J=12.5,3.8 \mathrm{~Hz}, 1$ $\mathrm{H}, 5-\mathrm{H}_{\text {ax. }}$ ), 4.03 (ddd, $J=12.5,3.0,1.8 \mathrm{~Hz}, 1 \mathrm{H}, 5-\mathrm{H}_{\text {equ. }}$ ), 4.18 (dddt, $J=10.8,5.5,3.4,1.6 \mathrm{~Hz}, 1 \mathrm{H}$, 1-H), 4.52 (d, $\left.J=11.8 \mathrm{~Hz}, 1 \mathrm{H}, \mathrm{CH}_{2}-\mathrm{Ph}\right), 4.54$ (d, $\left.J=11.9 \mathrm{~Hz}, 1 \mathrm{H}, \mathrm{CH}_{2}-\mathrm{Ph}\right), 4.58$ (d, $J=11.8 \mathrm{~Hz}, 1$ $\left.\mathrm{H}, \mathrm{CH}_{2} \mathrm{Ph}\right), 4.62\left(\mathrm{~d}, J=11.9 \mathrm{~Hz}, 1 \mathrm{H}, \mathrm{CH}_{2}-\mathrm{Ph}\right), 5.09$ (dt, $\left.J=10.7,1.6 \mathrm{~Hz}, 1 \mathrm{H}, 7-\mathrm{H}\right), 5.23(\mathrm{dt}, J=$ 17.3, 1.6 Hz, $\left.1 \mathrm{H}, 7^{\prime}-\mathrm{H}\right), 5.84$ (ddd, $\left.J=17.3,10.7,5.5 \mathrm{~Hz}, 1 \mathrm{H}, 6-\mathrm{H}\right), 7.27-7.37$ (m, $10 \mathrm{H}$, arom. H); ${ }^{13} \mathrm{C}$ NMR (125 MHz, $\left.\mathrm{CD}_{2} \mathrm{Cl}_{2}\right): \delta 32.5$ (t, C-2), 65.5 (t, C-5), 71.4, 71.5 (2t, $\left.\mathrm{CH}_{2}-\mathrm{Ph}\right), 72.8(\mathrm{~d}, \mathrm{C}-1)$, 73.2 (d, C-3), 73.5 (d, C-4), 114.9 (t, C-7), 127.9, 128.0, 128.1, 128.2, 128.6, 128.8 (6d, C-arom.), 139.1, 139.2 (2s, C-arom.), 139.7 (d, C-6); IR (film) $\tilde{v}=3030,2959,2921,2856,1453,1353,1261$, 1082, 919, 801, 737, $698 \mathrm{~cm}^{-1}$; HRMS (ESI-Q-TOF): $\mathrm{m} / z$ calcd for $\mathrm{C}_{21} \mathrm{H}_{25} \mathrm{O}_{3}[\mathrm{M}-\mathrm{H}]^{+}: 325.1804$ found 325.1786 .

\section{References}

(1) (a) Shull, B. K.; Wu, Z.; Koreeda, M. A Convenient, Highly Efficient One-Pot Preparation of Peracetylated Glycals From Reducing Sugars. J. Carbohydr. Chem. 1996, 15, 955-964. (b) Chmielewski, M.; Fokt, I.; Grodner, J.; Grynkiewicz G.; Szeja W. An Improved Synthesis and NMR Spectra of Benzylated Glycals. J. Carbohydr. Chem. 1989, 8, 735-741.

(2) (a) Timmers, C. M.; Leeuwenburgh, M. A.; Verheijen, J. C.; van der Marel, G. A.; van Boom, J. H. Rhodium(II) Catalyzed Asymmetric Cyclopropanation of Glycals with Ethyl Diazoacetate. Tetrahedron Asymm. 1996, 7, 49-52. (b) Hoberg, J. O.; Claffey, D. J. Cyclopropanation of Unsaturated Sugars with Ethyl Diazoacetate. Tetrahedron Lett. 1996, 37, 2533-2536.

(3) Haveli, S. D.; Sridhar, P. R.; Suguna, C.; Chandrasekaran, S. Efficient Synthesis of Fused Perhydrofuro[2,3-b]pyrans (and Furans) by Ring Opening of 1,2-Cyclopropanated Sugar Derivatives. Org. Lett. 2007, 9, 1331-1334. 


\section{NMR spectra}

\section{8a. Cyclopropanes 7}

${ }^{1} \mathrm{H} \mathrm{NMR}\left(600 \mathrm{MHz}, \mathrm{CD}_{2} \mathrm{Cl}_{2}\right)$
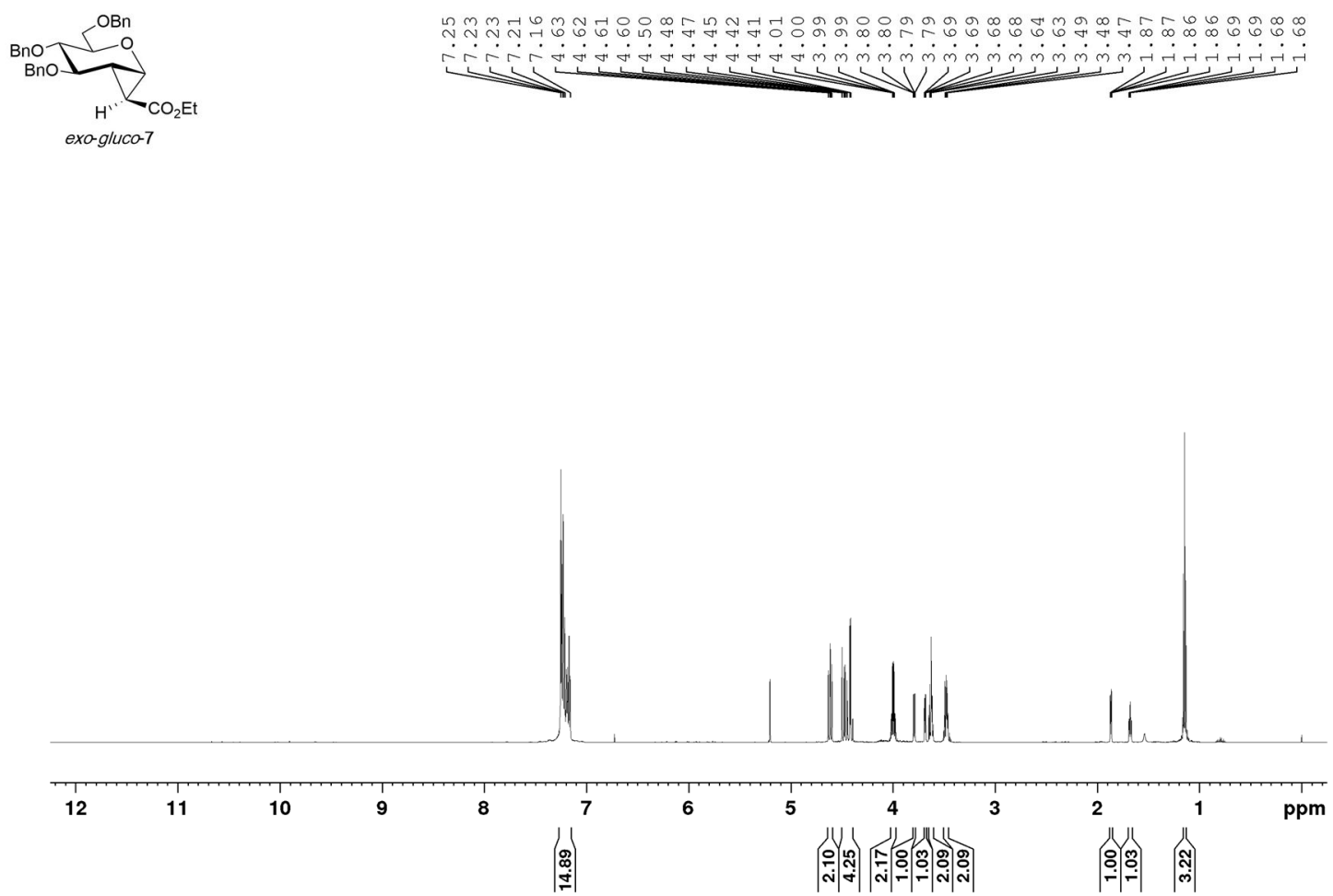

${ }^{13} \mathrm{C} \mathrm{NMR}\left(150 \mathrm{MHz}, \mathrm{CD}_{2} \mathrm{Cl}_{2}\right)$
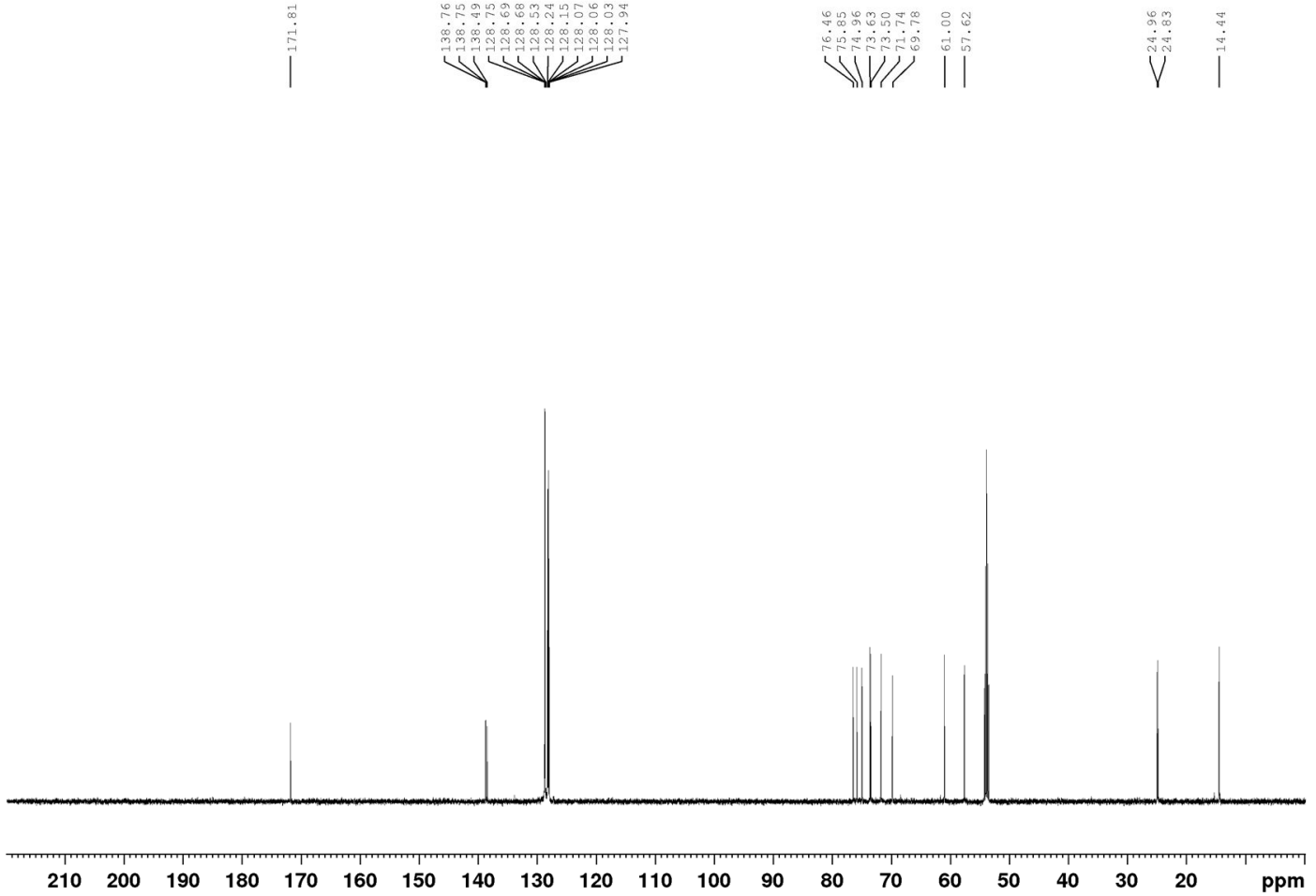
${ }^{1} \mathrm{H} \mathrm{NMR}\left(500 \mathrm{MHz}, \mathrm{CD}_{2} \mathrm{Cl}_{2}\right)$

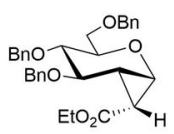

$\mathrm{EtO}_{2} \mathrm{C}$ ".'-

endo-gluco-7
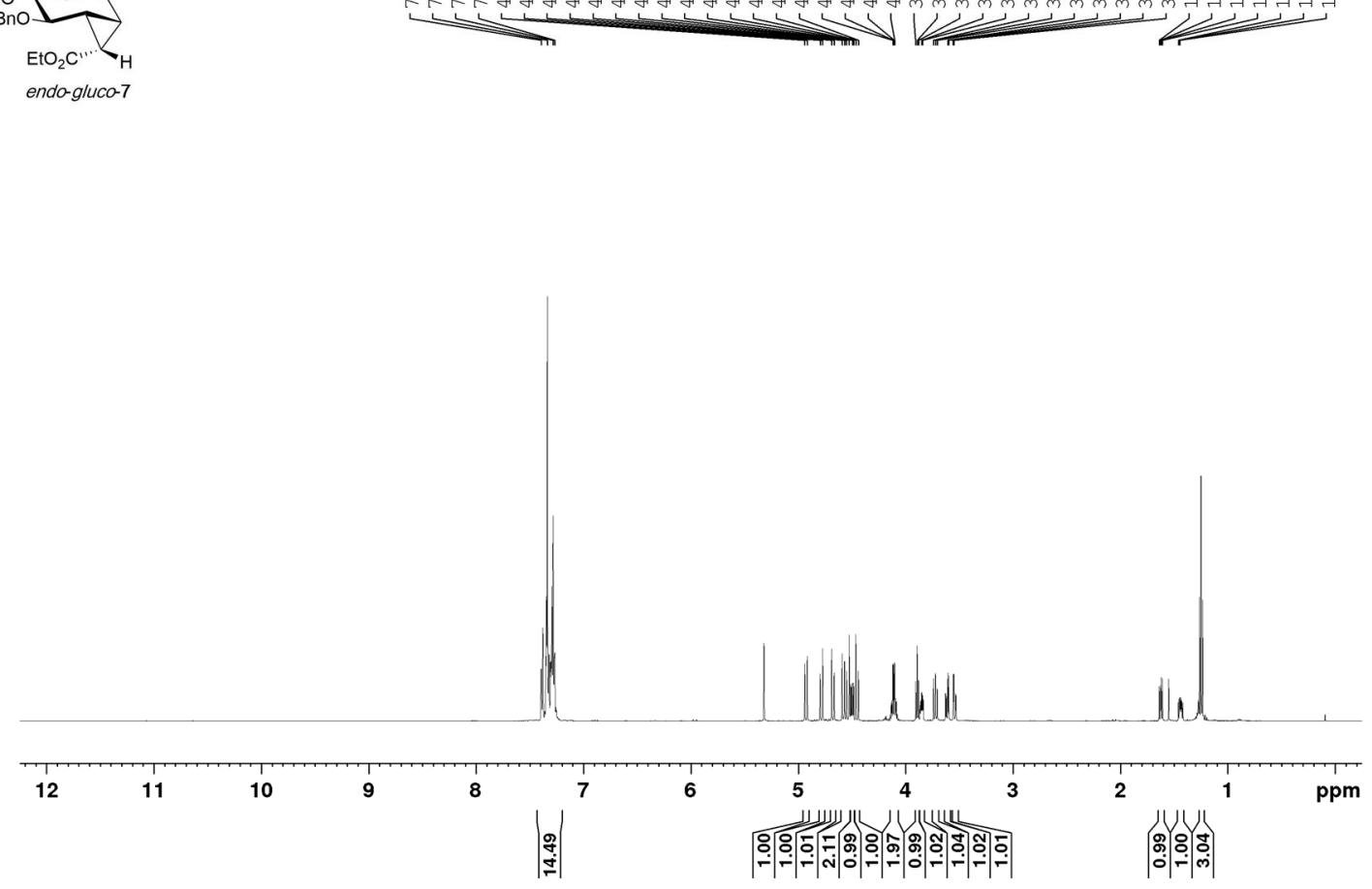

${ }^{13} \mathrm{C}$ NMR $\left(125 \mathrm{MHz}, \mathrm{CD}_{2} \mathrm{Cl}_{2}\right)$

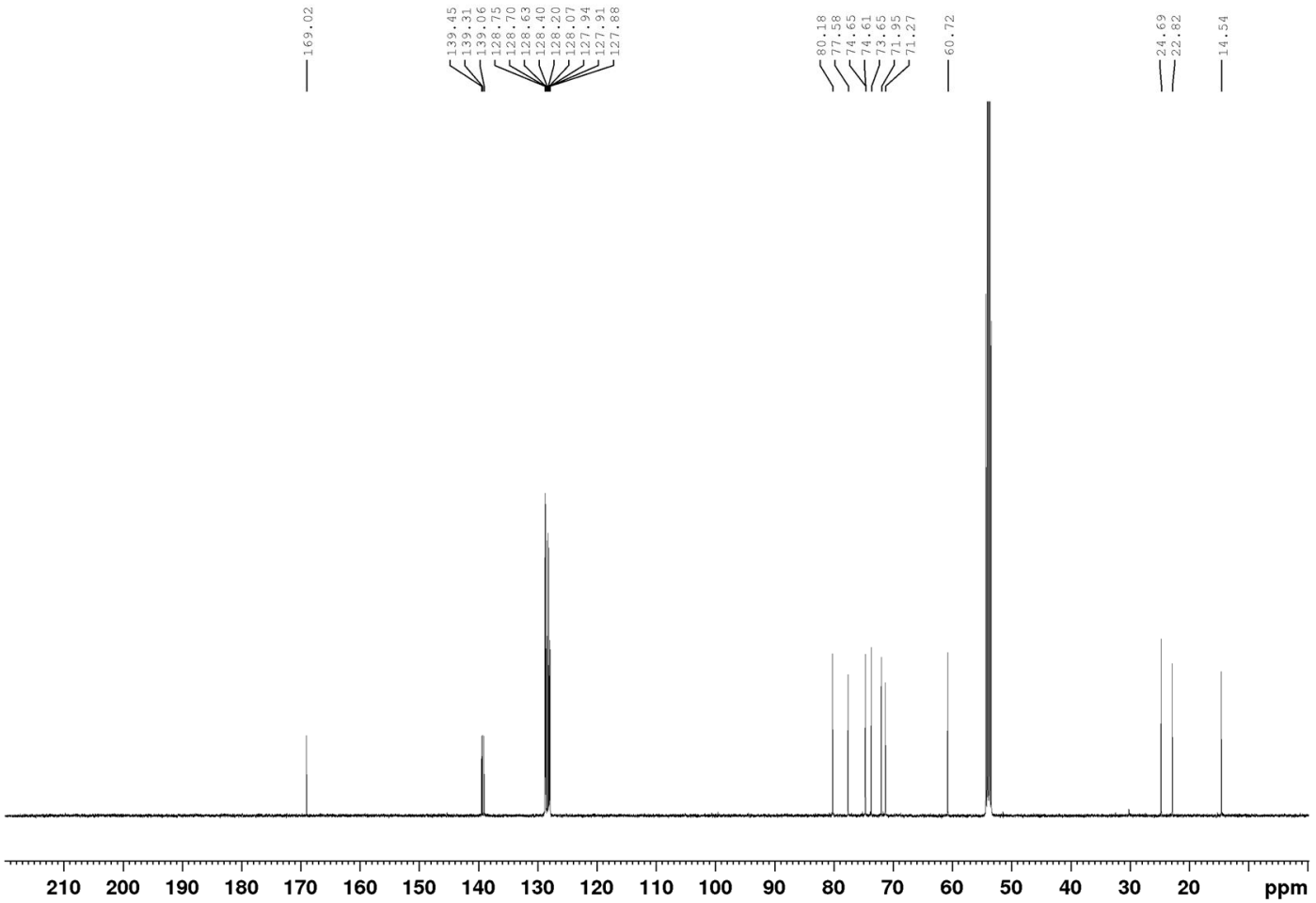


${ }^{1} \mathrm{H}$ NMR $\left(500 \mathrm{MHz}, \mathrm{CD}_{2} \mathrm{Cl}_{2}\right)$

$$
\text { manno-7 }
$$

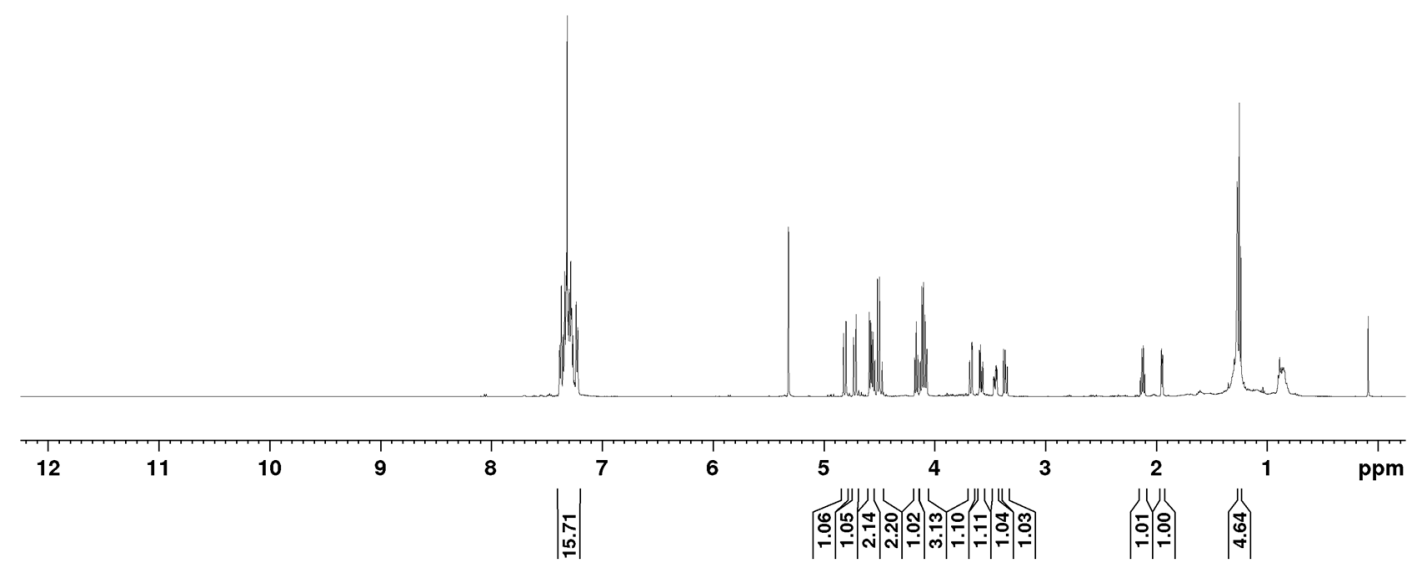

${ }^{13} \mathrm{C}$ NMR $\left(125 \mathrm{MHz}, \mathrm{CD}_{2} \mathrm{Cl}_{2}\right)$

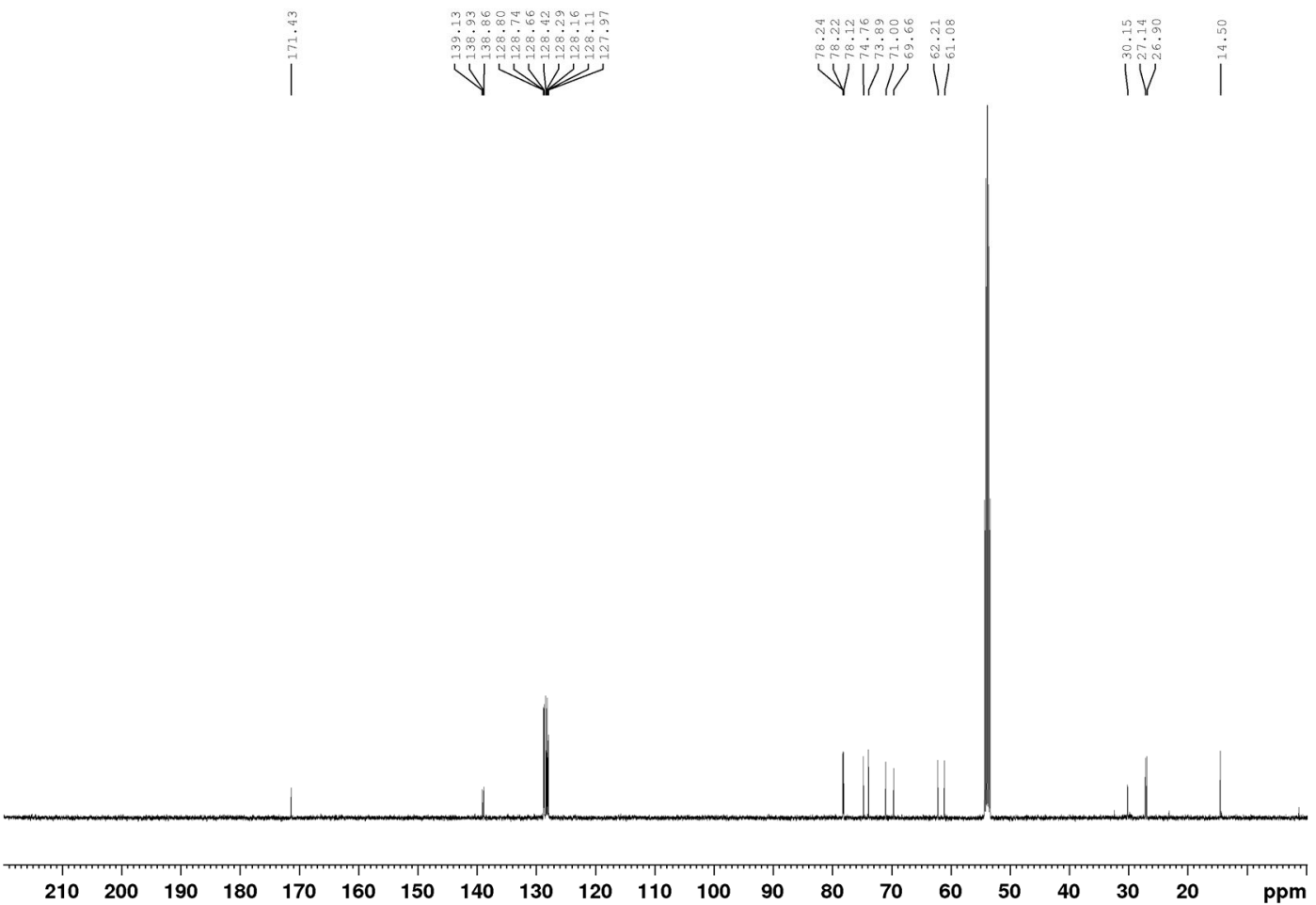


${ }^{1} \mathrm{H}$ NMR (600 MHz, $\mathrm{CD}_{2} \mathrm{Cl}_{2}$ )
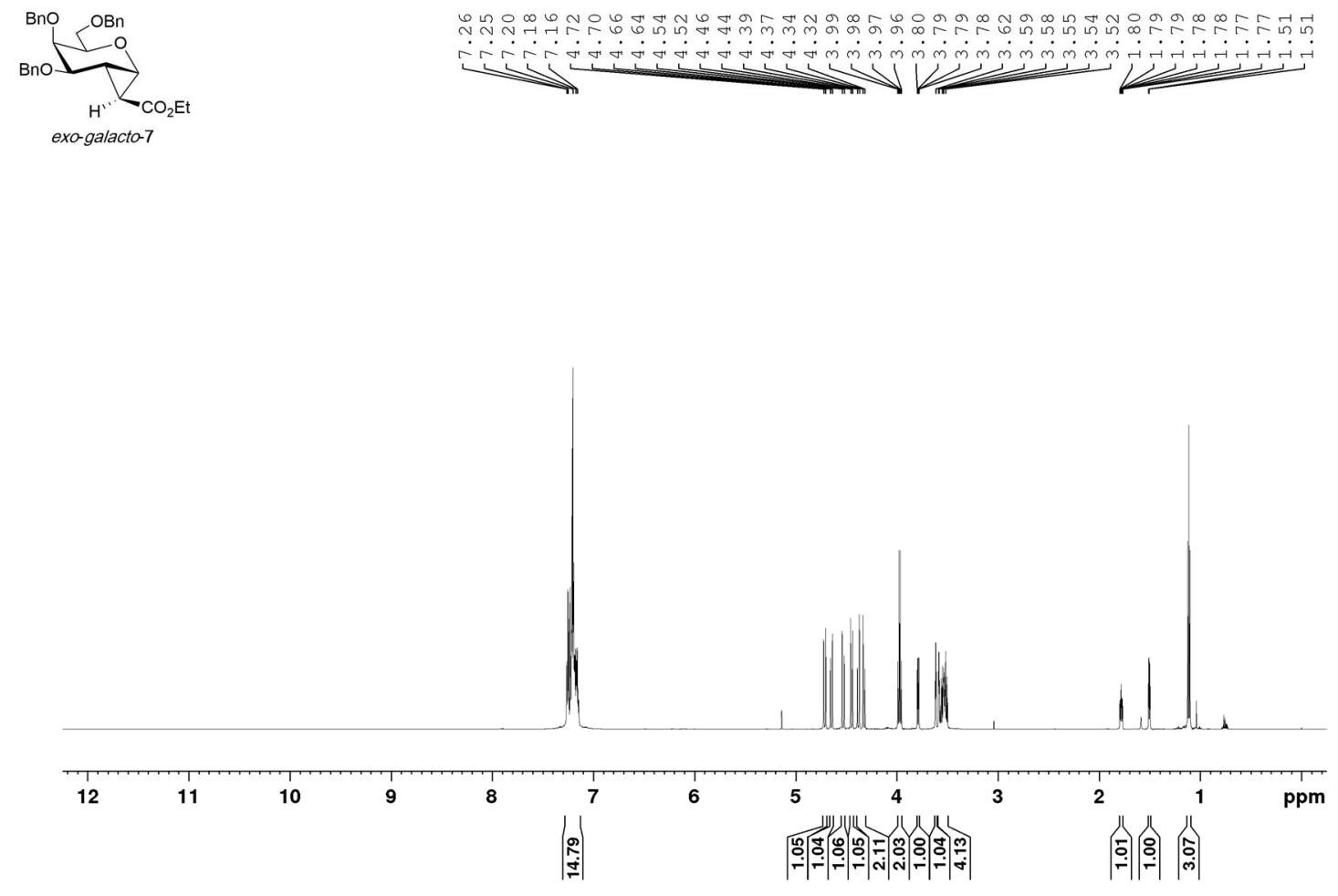

${ }^{13} \mathrm{C}$ NMR (150 MHz, $\mathrm{CD}_{2} \mathrm{Cl}_{2}$ )
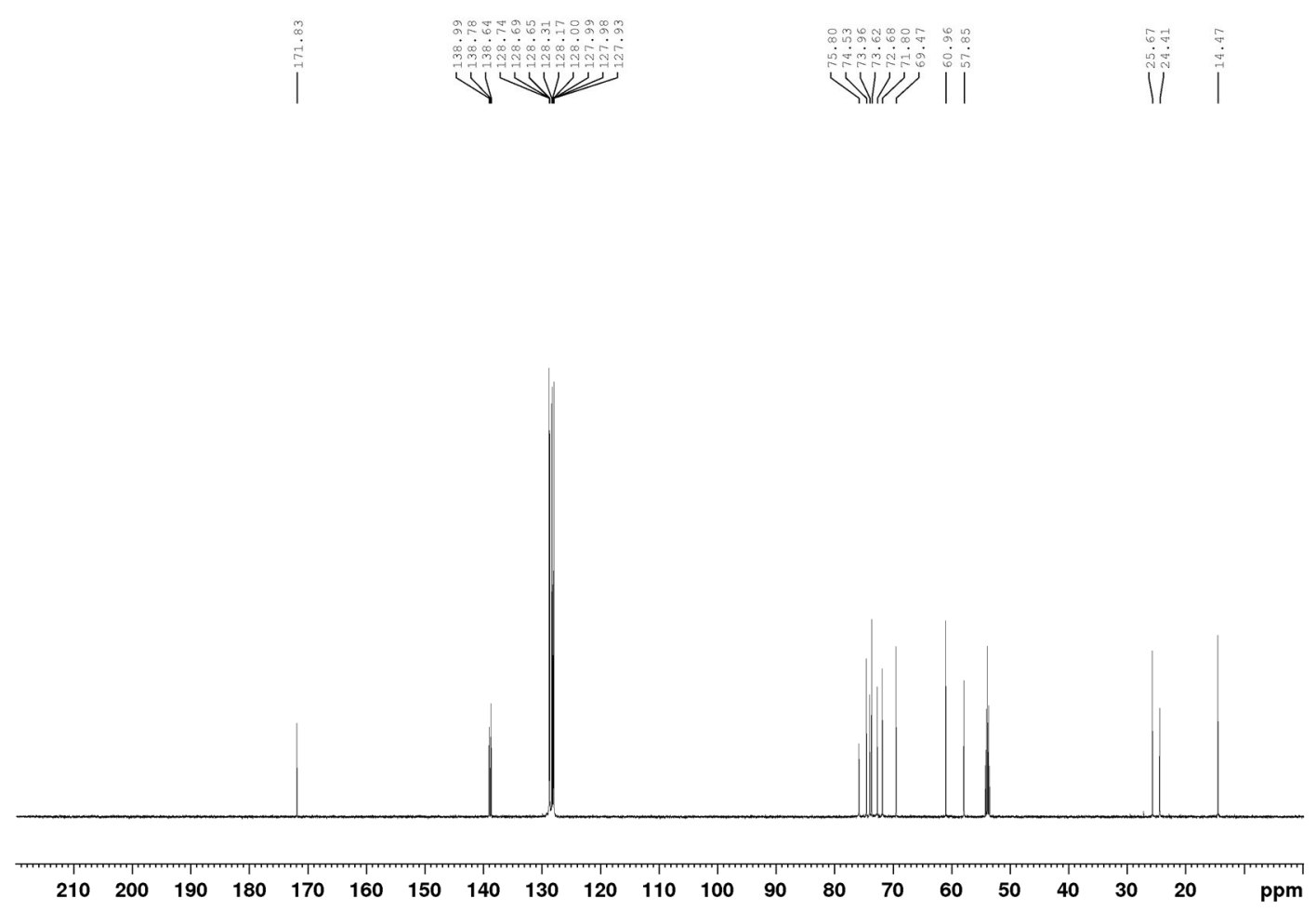

S27 
${ }^{1} \mathrm{H}$ NMR $\left(600 \mathrm{MHz}, \mathrm{CD}_{2} \mathrm{Cl}_{2}\right)$
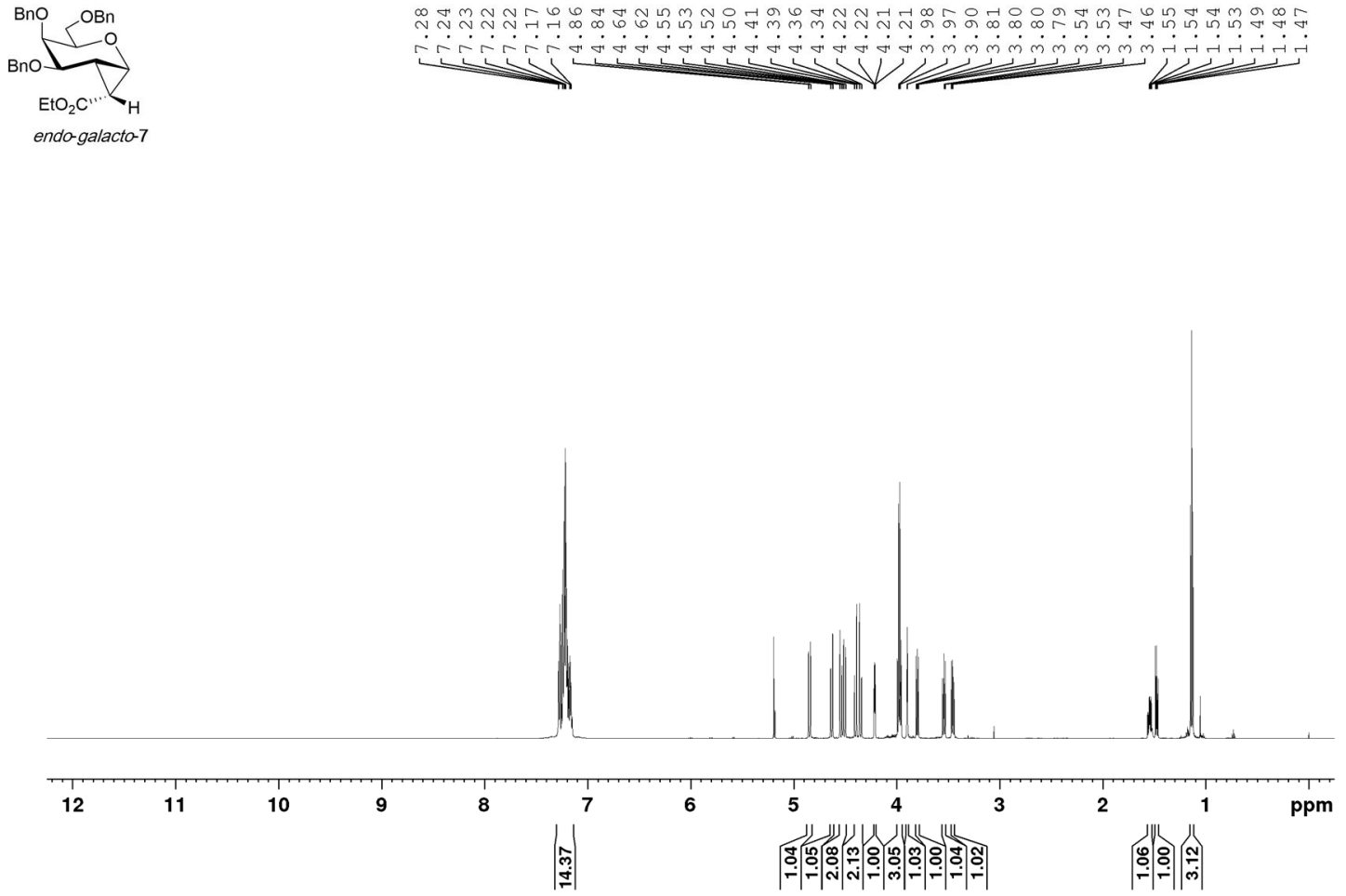

${ }^{13} \mathrm{C} \mathrm{NMR}\left(150 \mathrm{MHz}, \mathrm{CD}_{2} \mathrm{Cl}_{2}\right)$

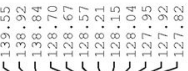

Vylj

$\sqrt{3}$

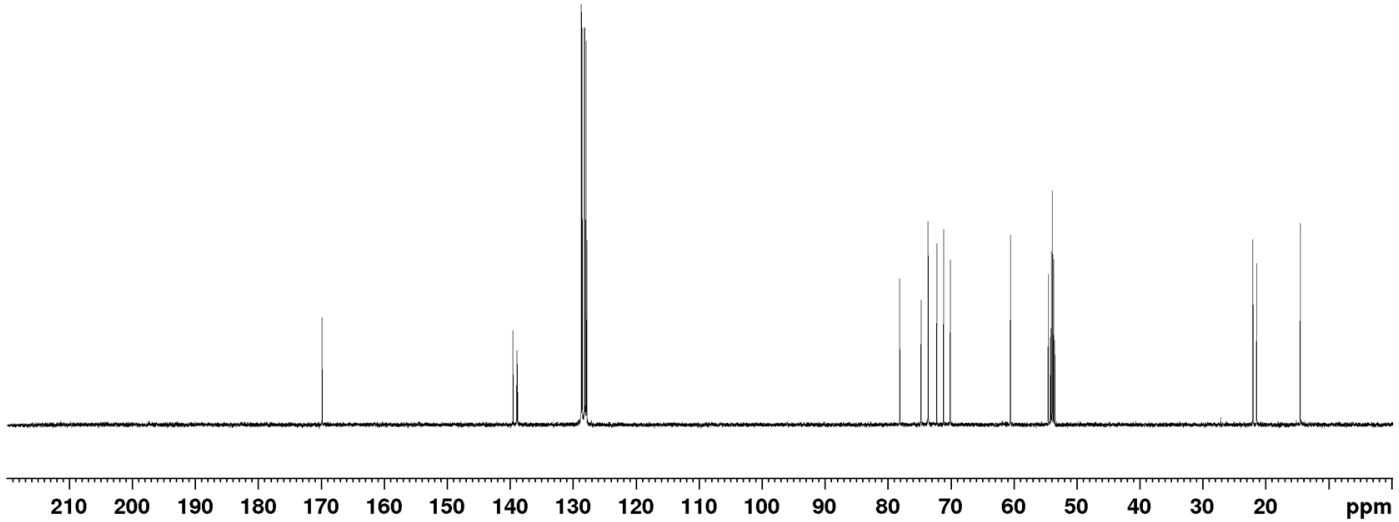


${ }^{1} \mathrm{H}$ NMR (600 MHz, $\left.\mathrm{CD}_{2} \mathrm{Cl}_{2}\right)$

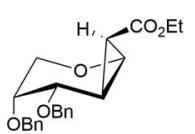

exo-arabino-7

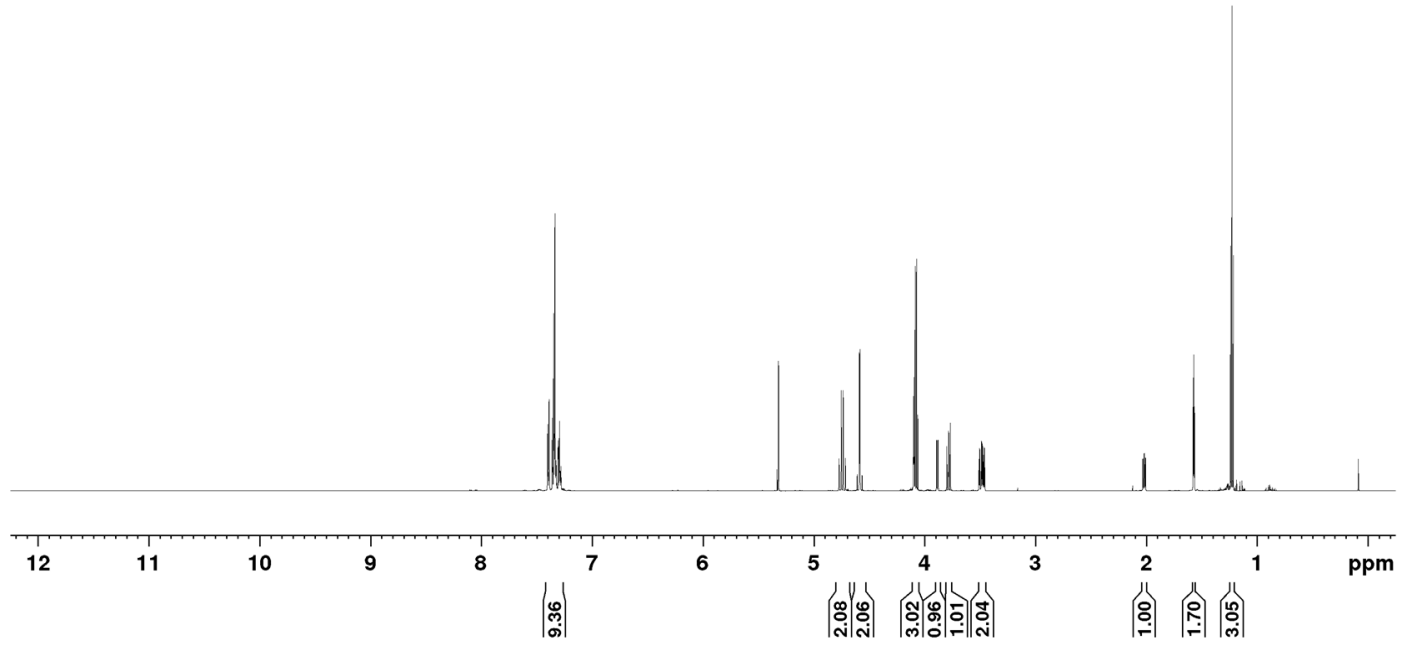

${ }^{13} \mathrm{C}$ NMR (125 MHz, $\left.\mathrm{CD}_{2} \mathrm{Cl}_{2}\right)$
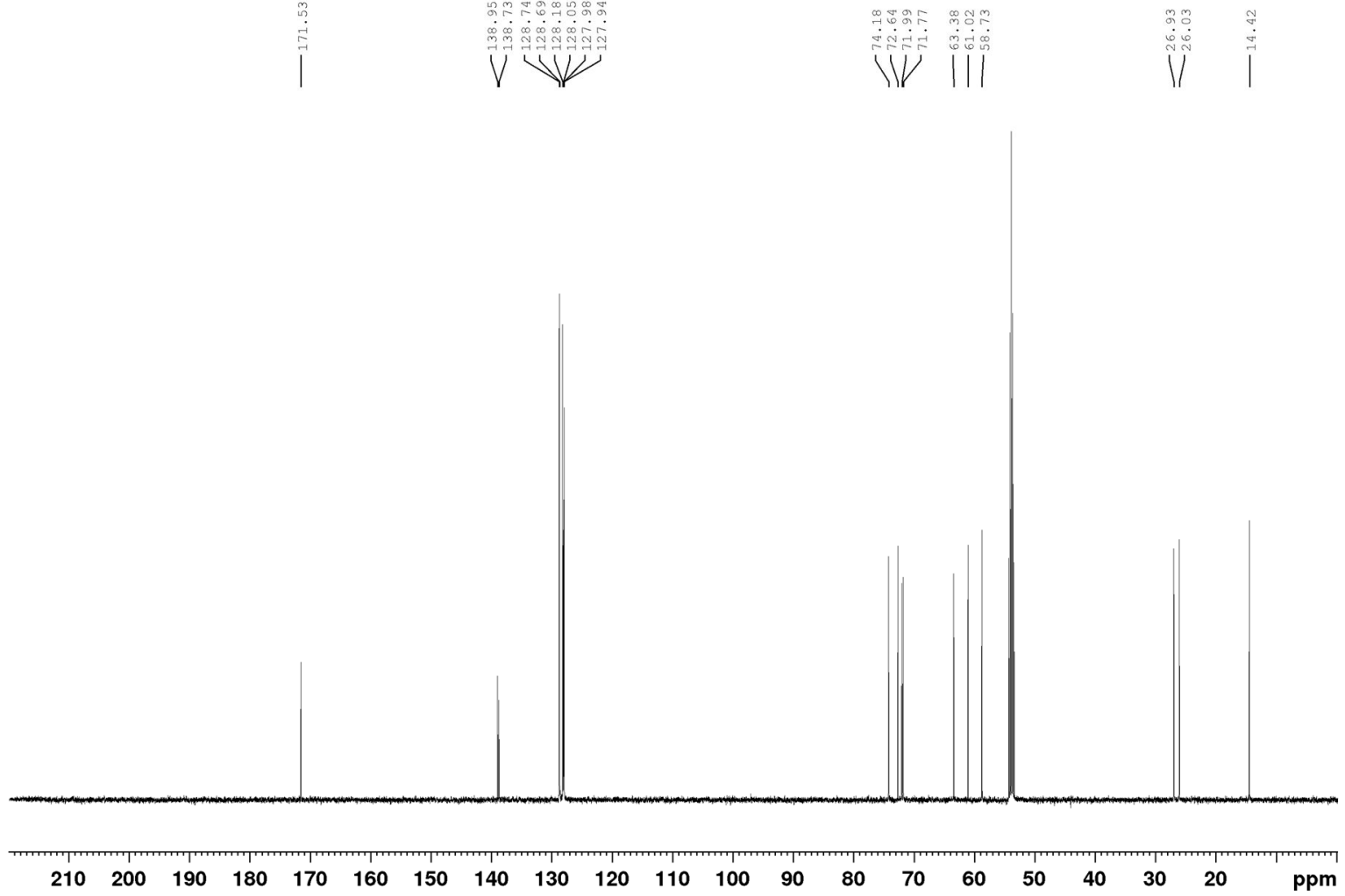
${ }^{1} \mathrm{H}$ NMR ( $500 \mathrm{MHz}, \mathrm{CD}_{2} \mathrm{Cl}_{2}$ )

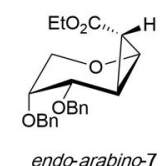

endo-arabino-7

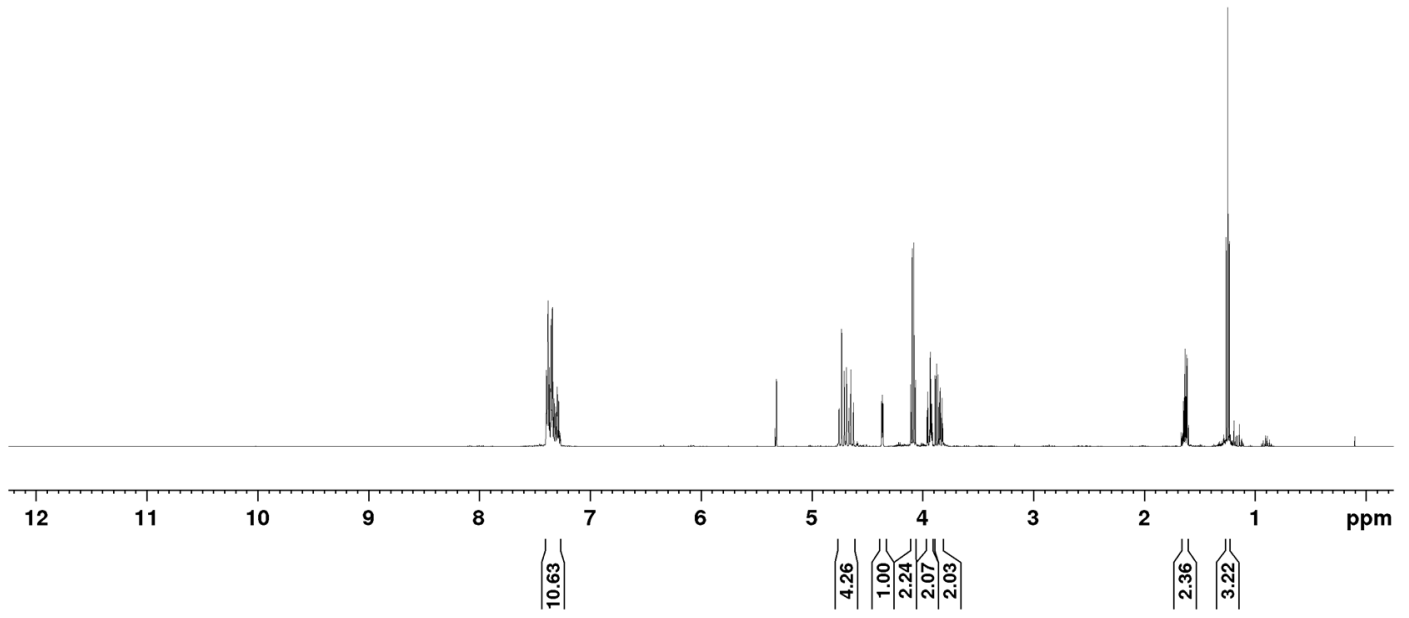

${ }^{13} \mathrm{C}$ NMR (125 MHz, $\mathrm{CD}_{2} \mathrm{Cl}_{2}$ )

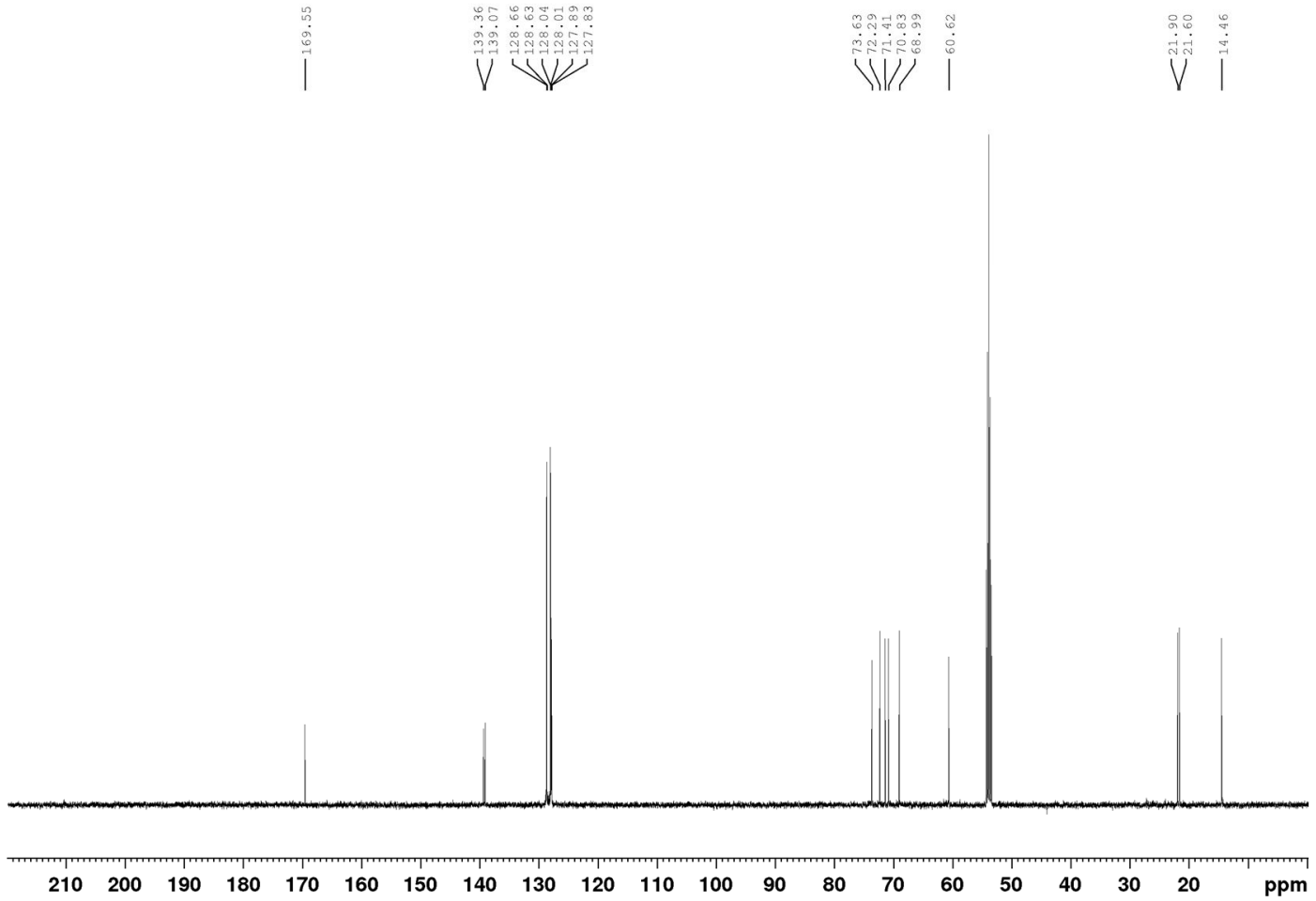


${ }^{1} \mathrm{H}$ NMR (500 MHz, $\mathrm{CD}_{2} \mathrm{Cl}_{2}$ )

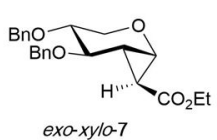

exo-xylo-7

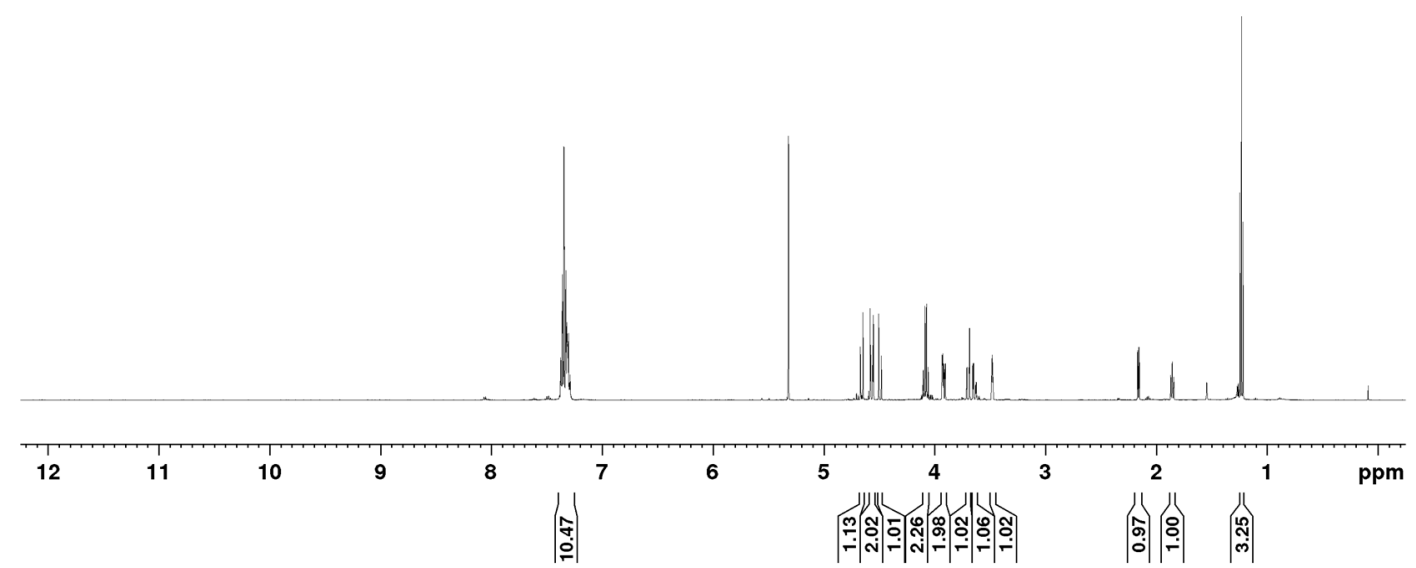

${ }^{13} \mathrm{C} \mathrm{NMR}\left(125 \mathrm{MHz}, \mathrm{CD}_{2} \mathrm{Cl}_{2}\right)$

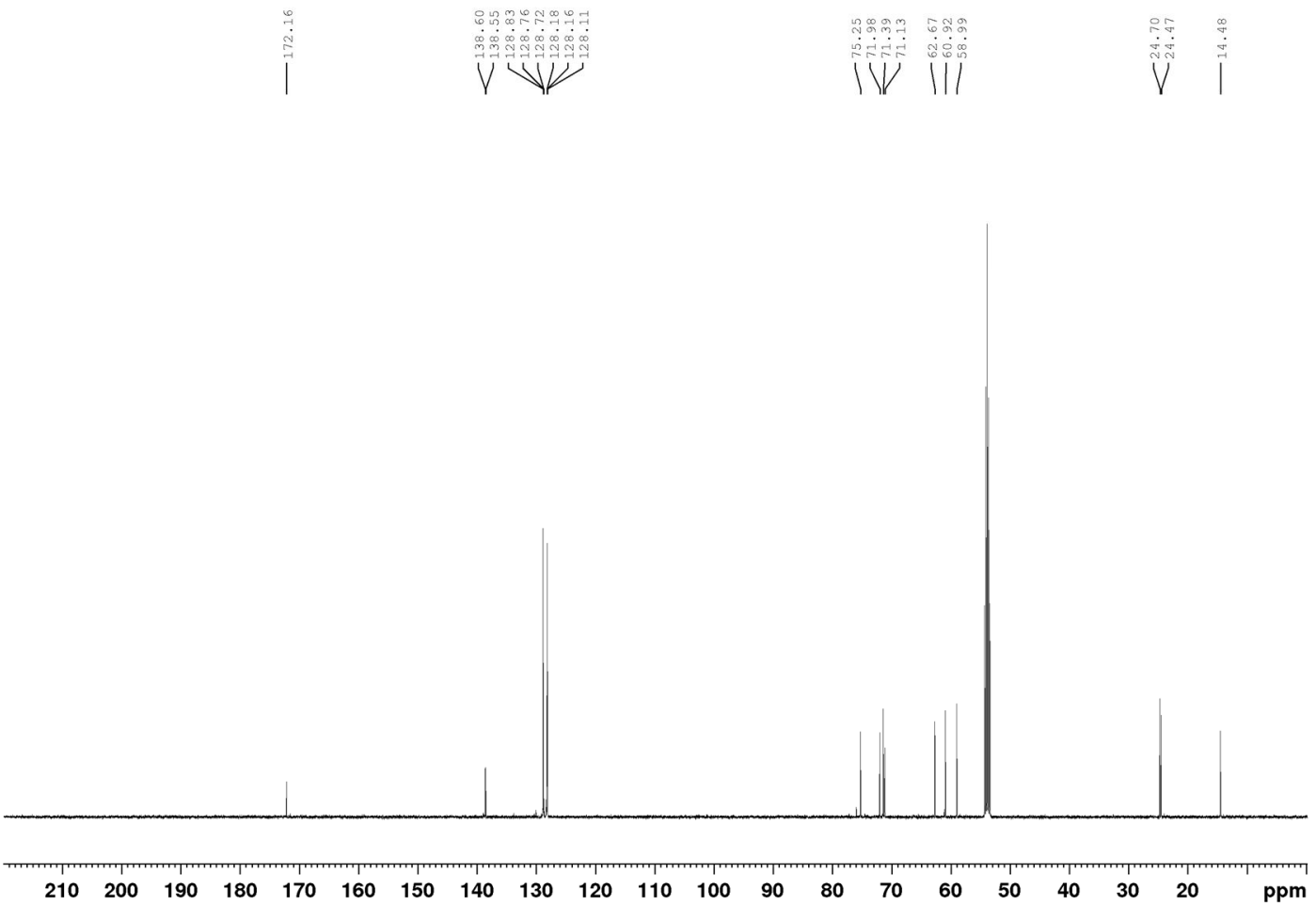


${ }^{1} \mathrm{H}$ NMR (500 MHz, $\mathrm{CD}_{2} \mathrm{Cl}_{2}$ )
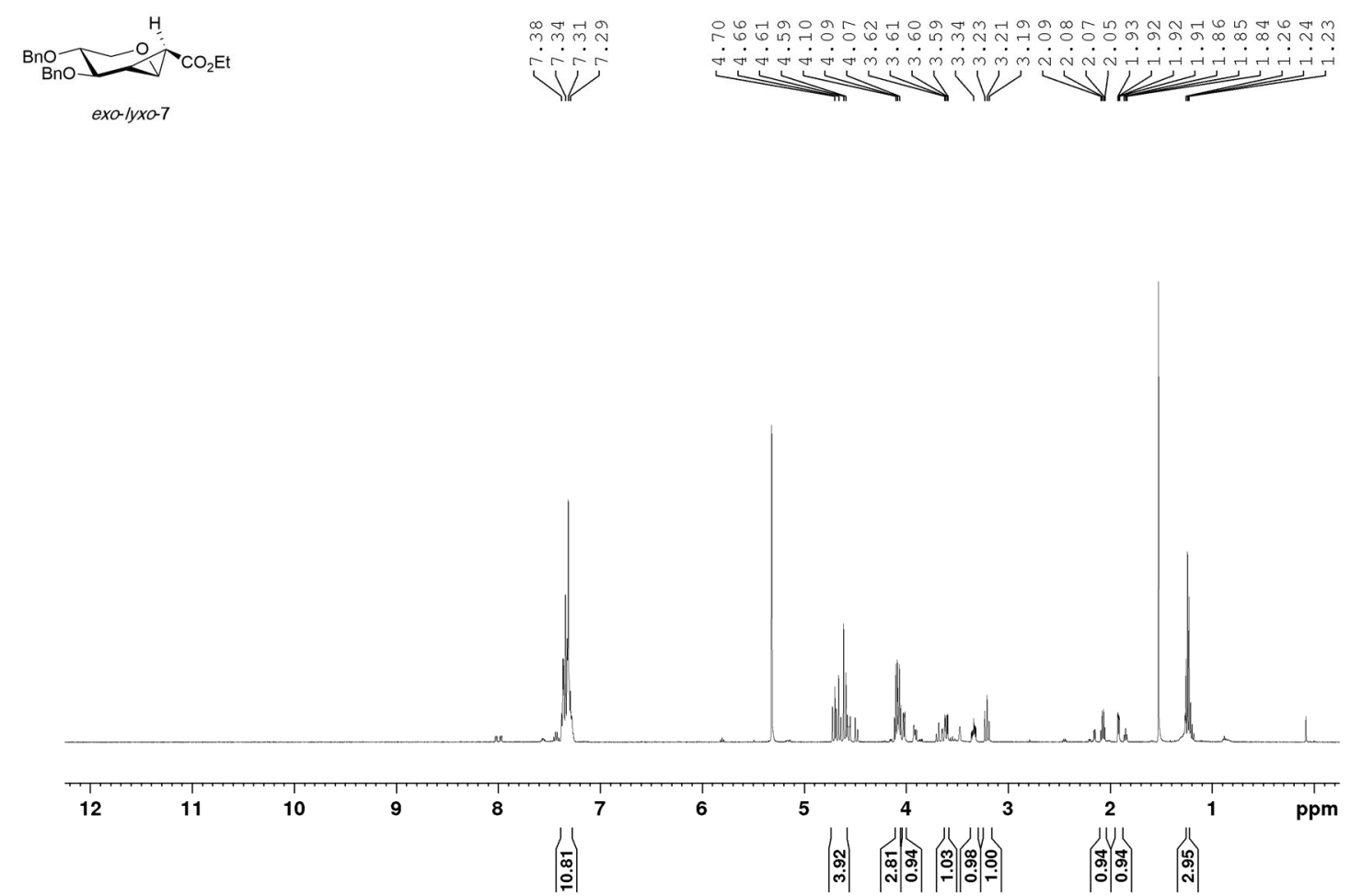

${ }^{13} \mathrm{C}$ NMR (125 MHz, $\left.\mathrm{CD}_{2} \mathrm{Cl}_{2}\right)$

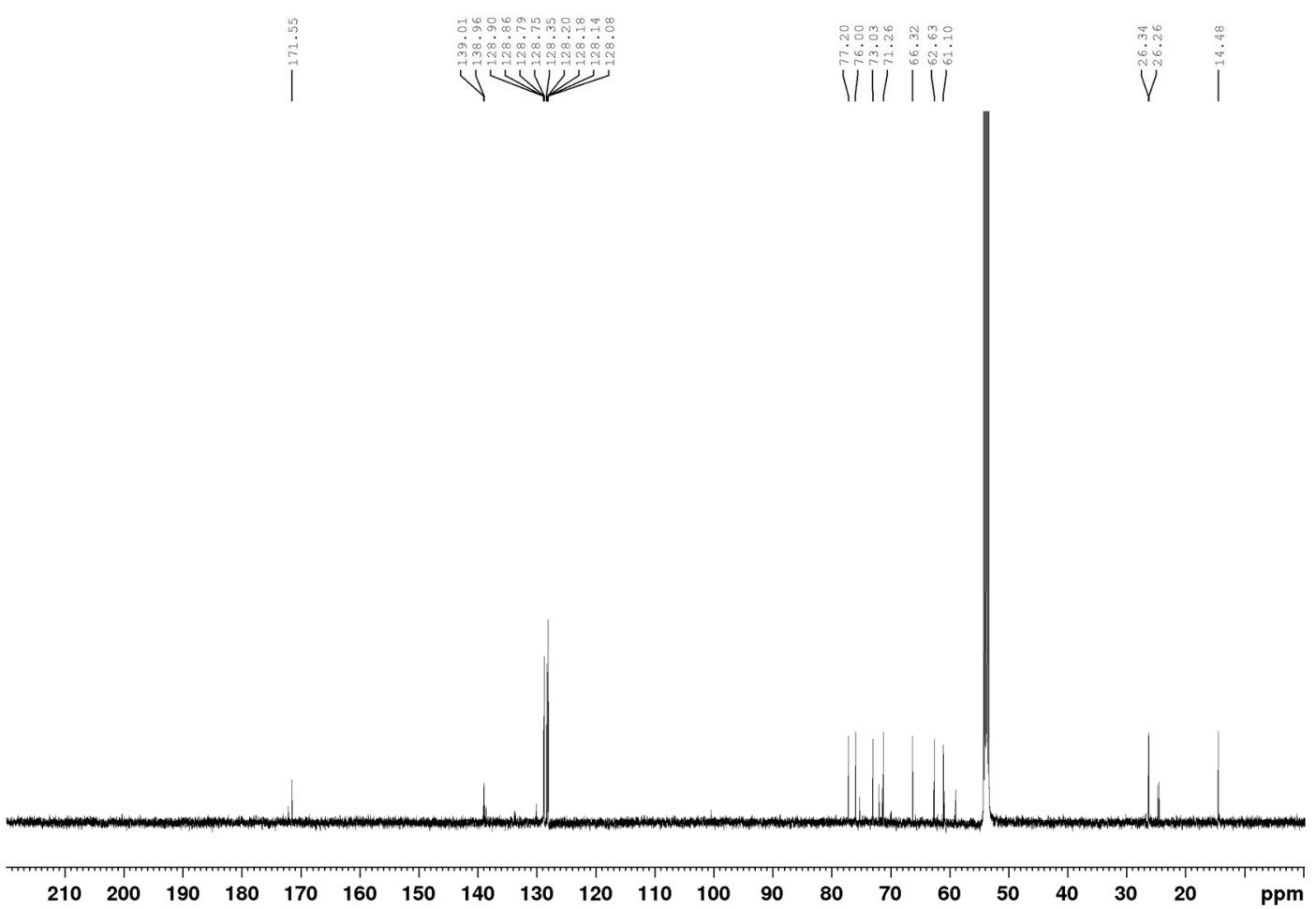




\section{8b. Alcohols 8}

${ }^{1} \mathrm{H}$ NMR $\left(600 \mathrm{MHz}, \mathrm{CD}_{2} \mathrm{Cl}_{2}\right)$
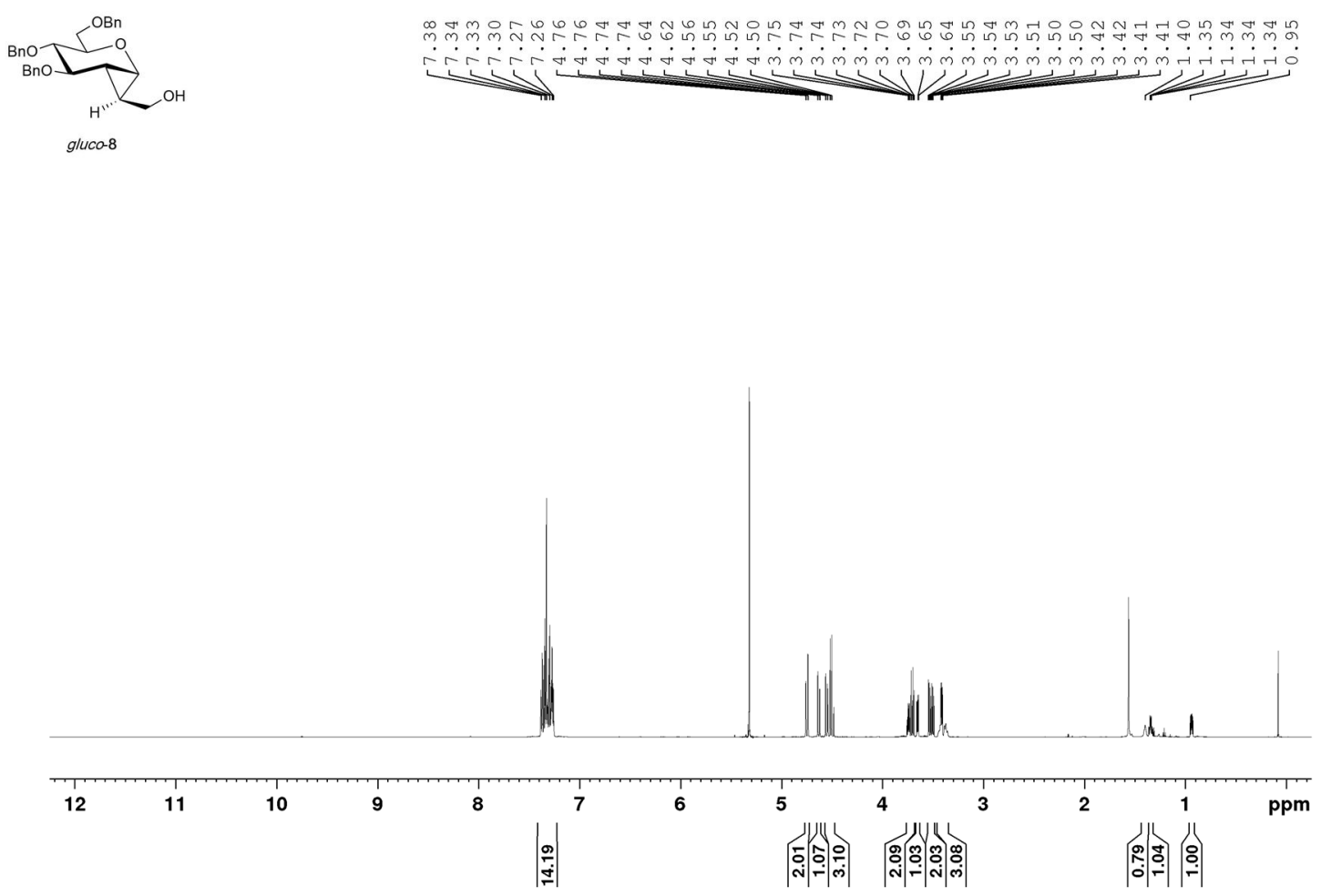

${ }^{13} \mathrm{C}$ NMR $\left(150 \mathrm{MHz}, \mathrm{CD}_{2} \mathrm{Cl}_{2}\right.$ )
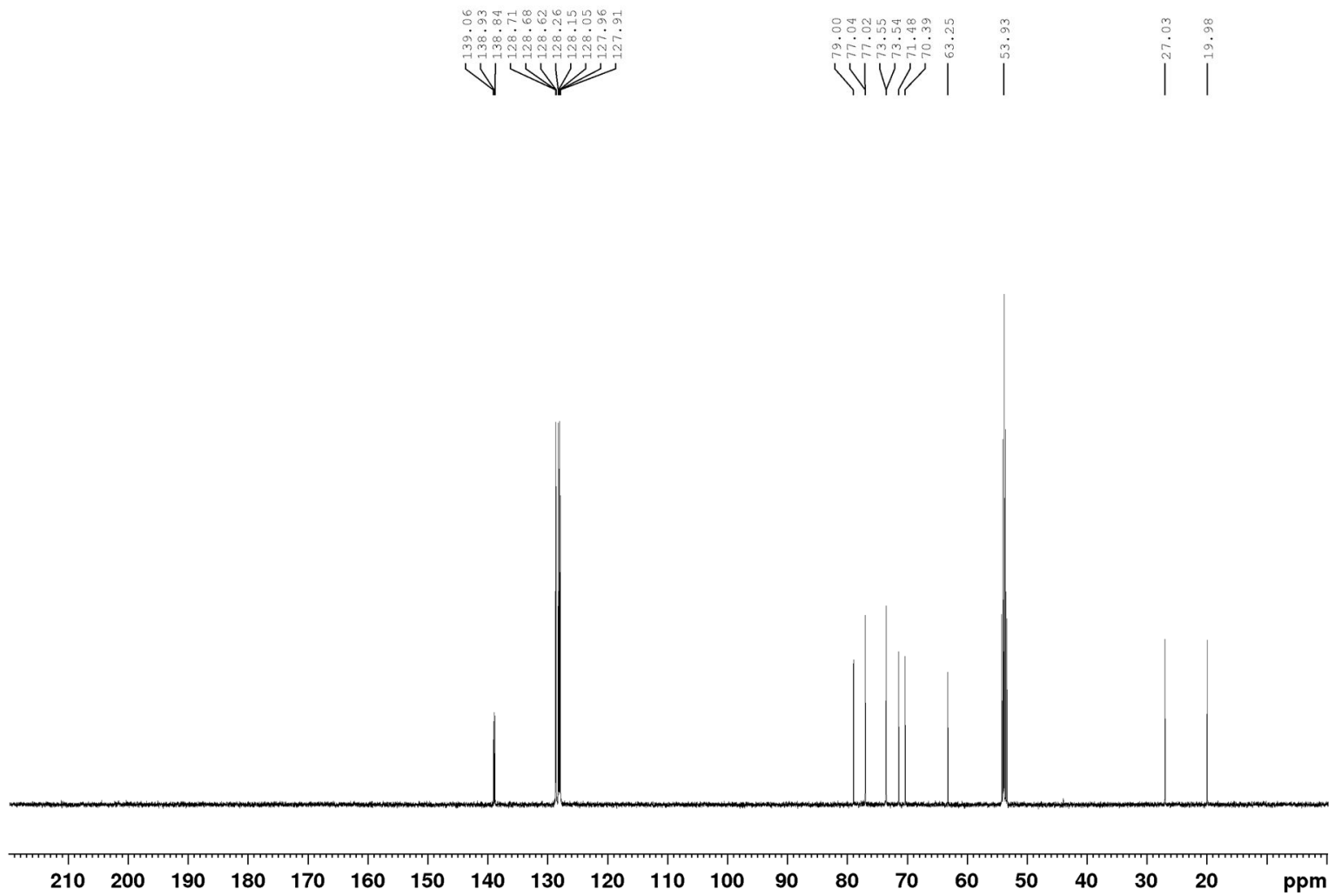
${ }^{1} \mathrm{H}$ NMR $\left(600 \mathrm{MHz}, \mathrm{CD}_{2} \mathrm{Cl}_{2}\right)$

$$
\text { manna-8 }
$$

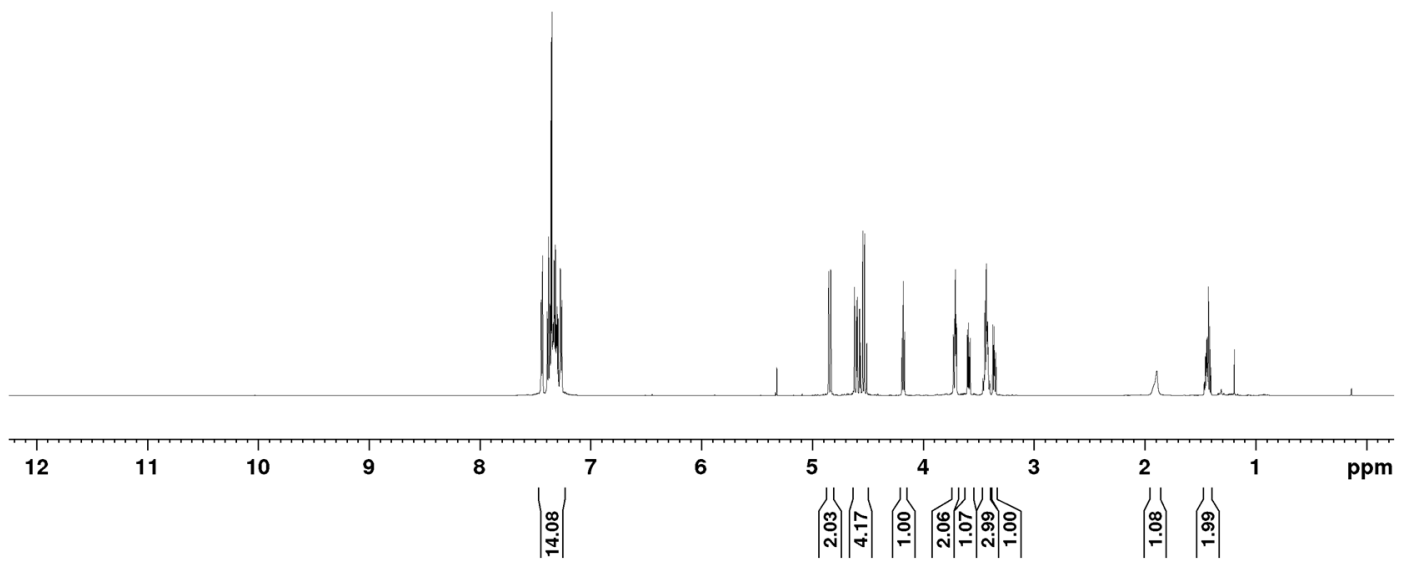

${ }^{13} \mathrm{C}$ NMR $\left(150 \mathrm{MHz}, \mathrm{CD}_{2} \mathrm{Cl}_{2}\right)$

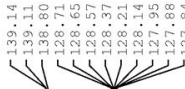

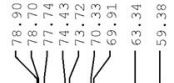

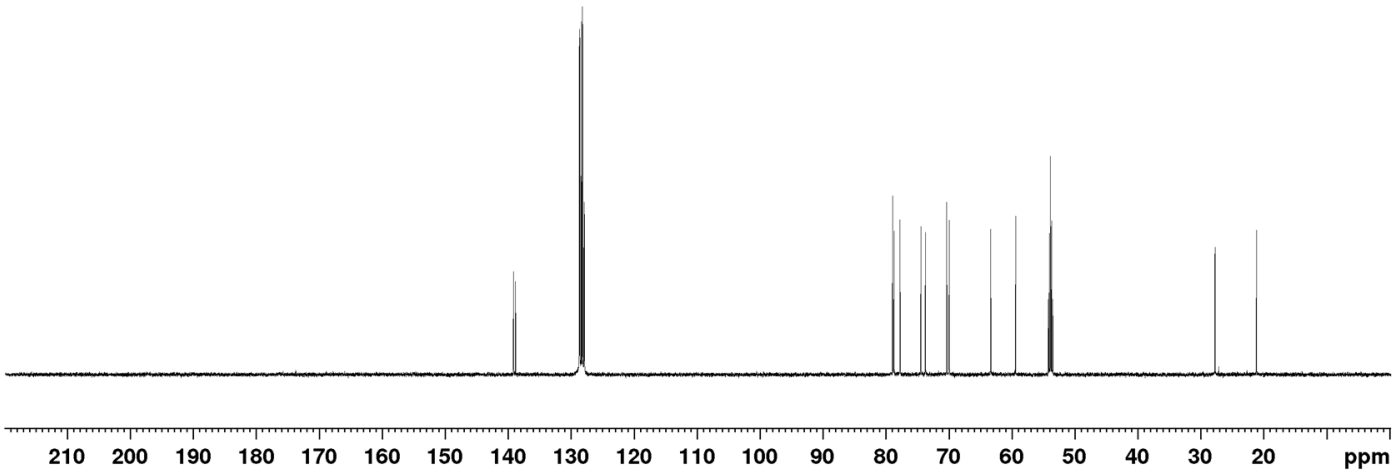


${ }^{1} \mathrm{H}$ NMR (600 MHz, $\mathrm{CD}_{2} \mathrm{Cl}_{2}$ )
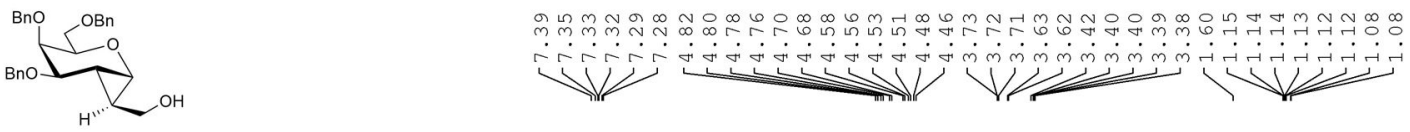

galacto-8

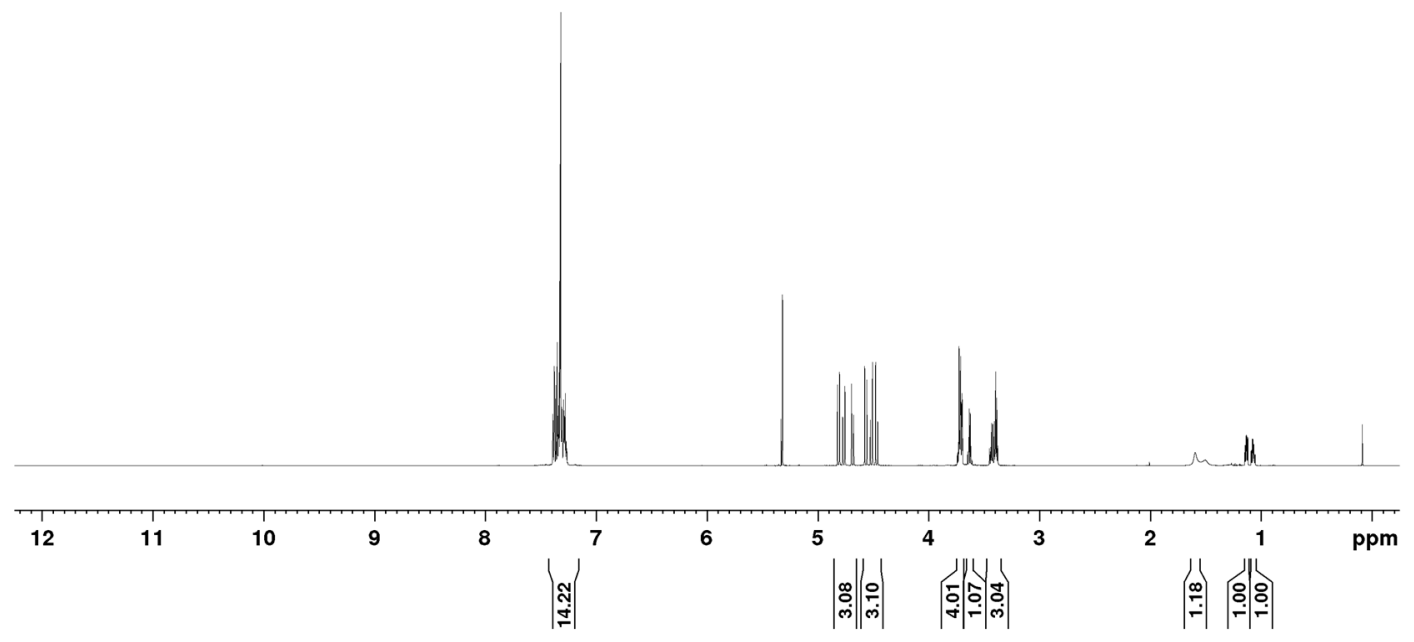

${ }^{13} \mathrm{C}$ NMR (125 MHz, $\mathrm{CD}_{2} \mathrm{Cl}_{2}$ )
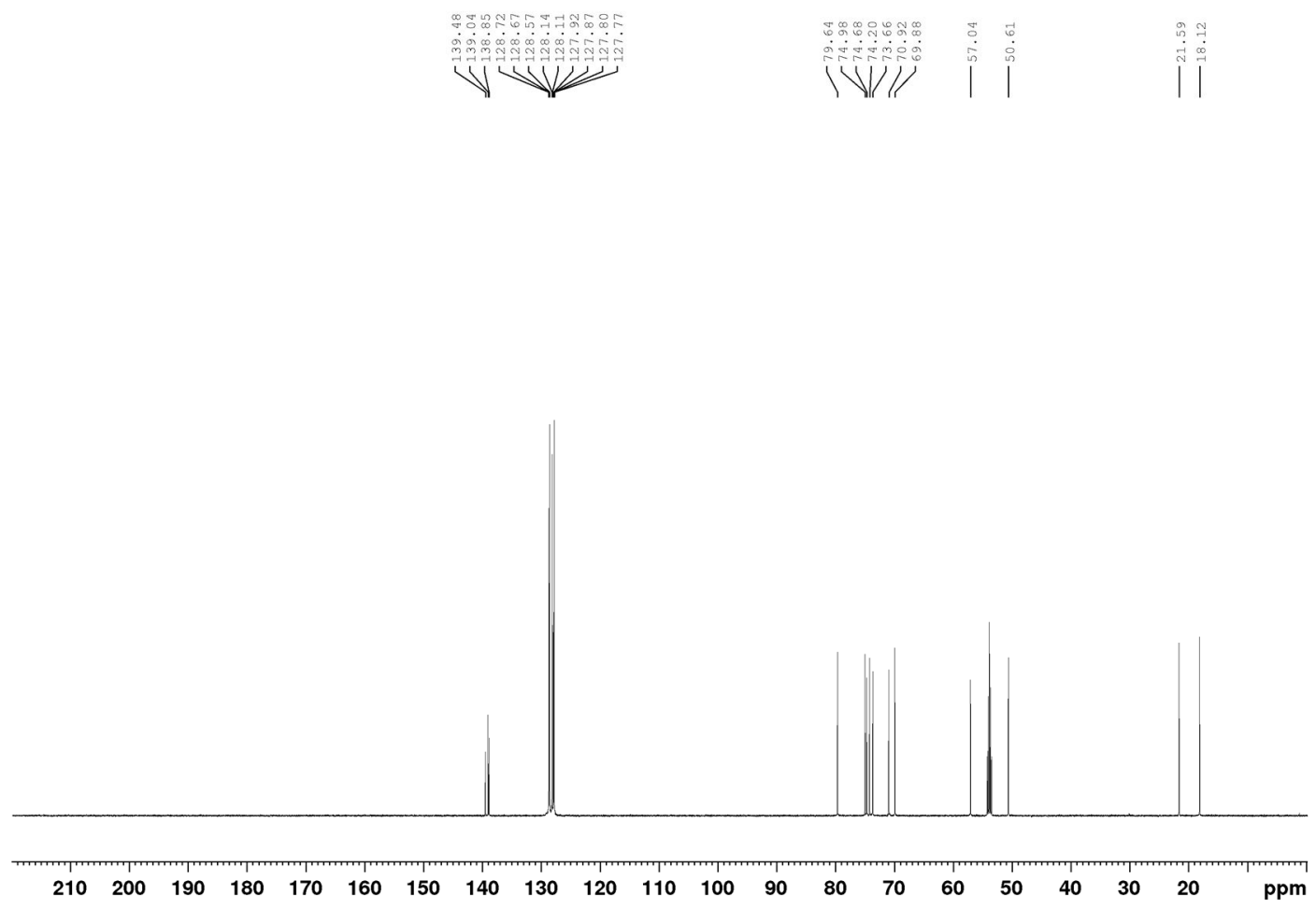

S35 
${ }^{1} \mathrm{H}$ NMR $\left(500 \mathrm{MHz}, \mathrm{CD}_{2} \mathrm{Cl}_{2}\right)$

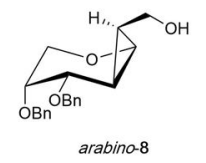

arabina 8
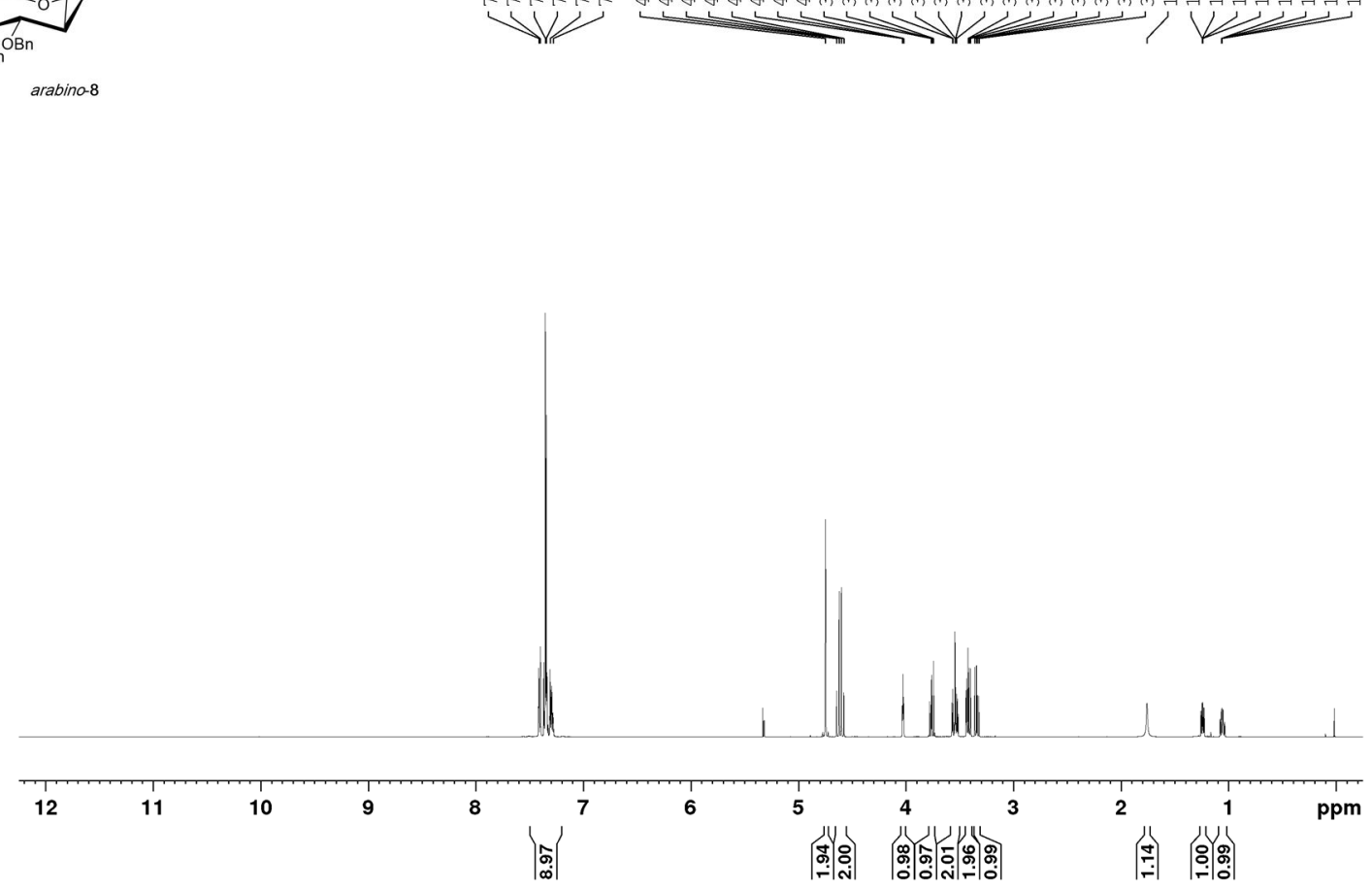

${ }^{13} \mathrm{C}$ NMR $\left(125 \mathrm{MHz}, \mathrm{CD}_{2} \mathrm{Cl}_{2}\right)$
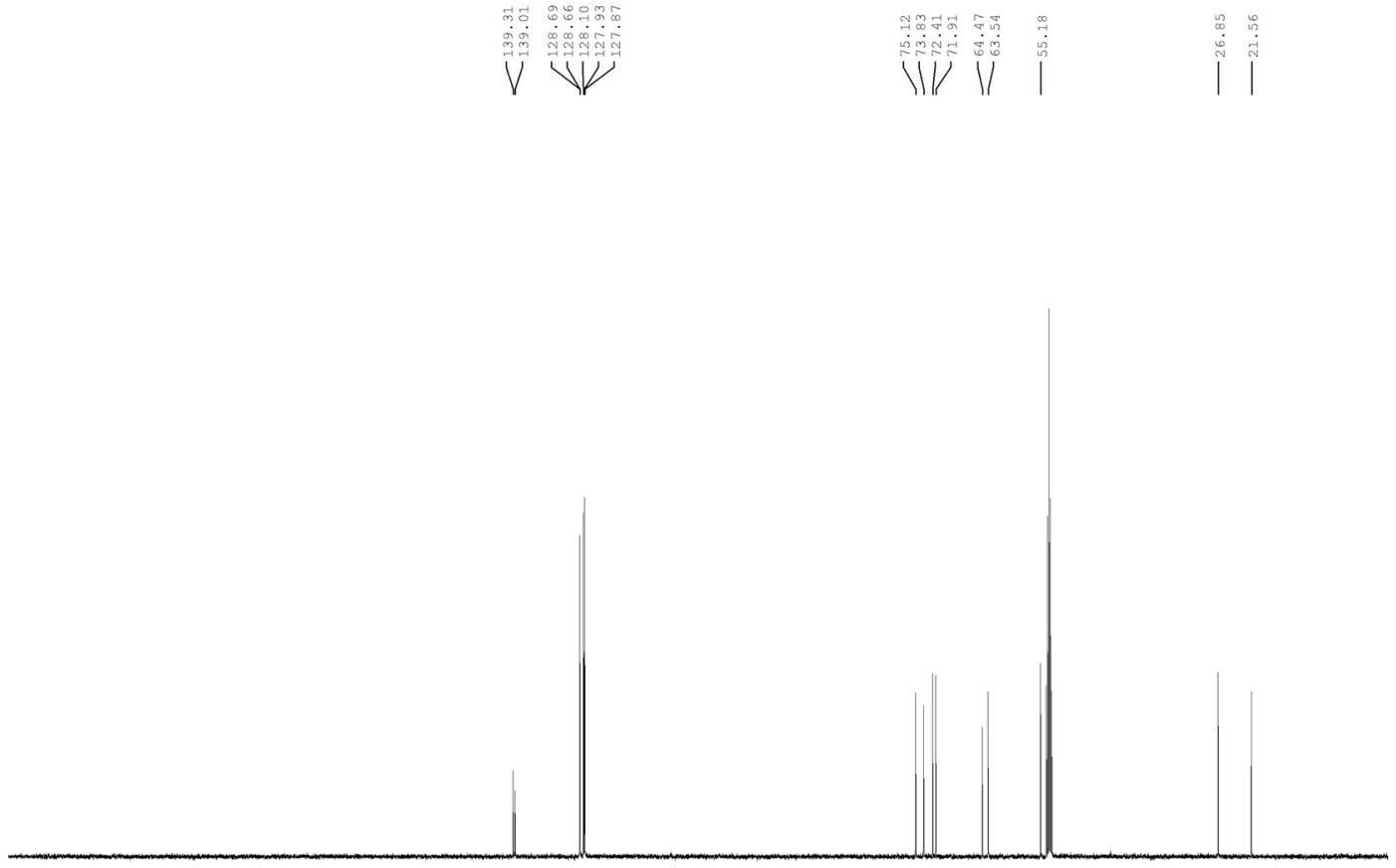

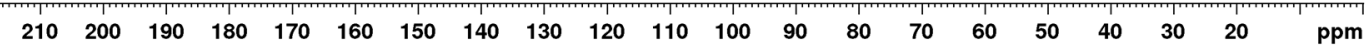


${ }^{1} \mathrm{H}$ NMR $\left(500 \mathrm{MHz}, \mathrm{CD}_{2} \mathrm{Cl}_{2}\right)$
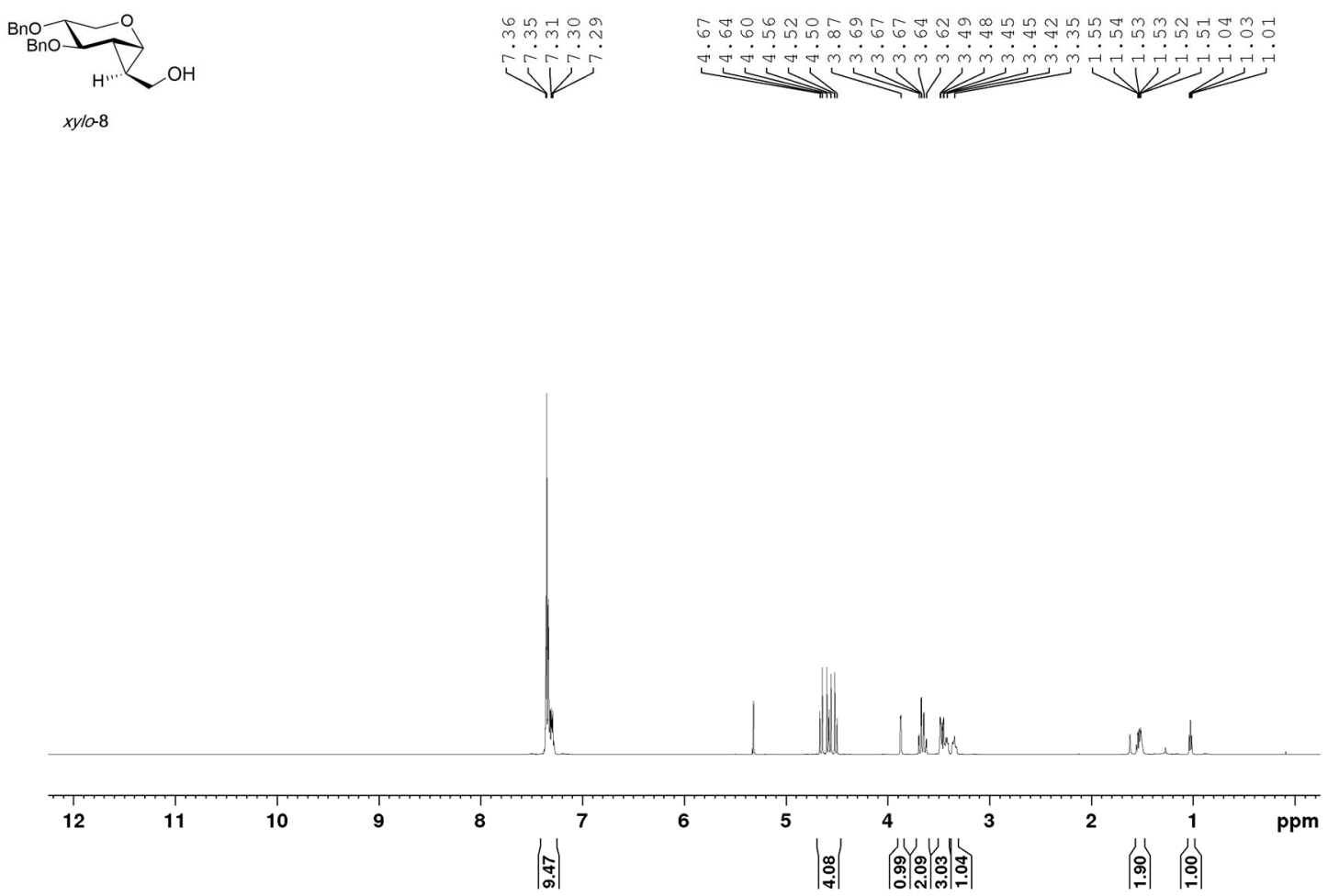

${ }^{13} \mathrm{C}$ NMR (125 MHz, $\mathrm{CD}_{2} \mathrm{Cl}_{2}$ )

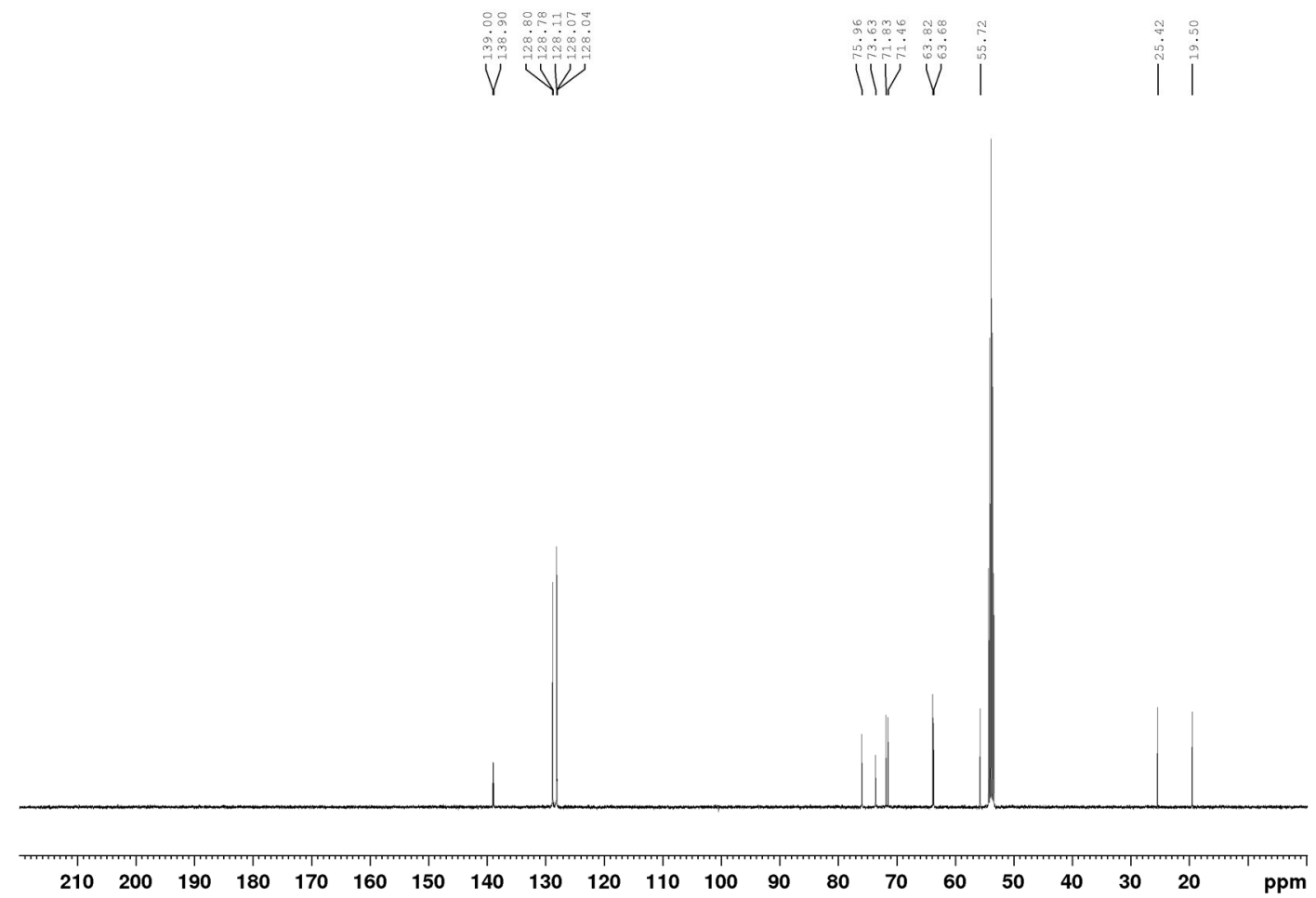


${ }^{1} \mathrm{H}$ NMR $\left(500 \mathrm{MHz}, \mathrm{CD}_{2} \mathrm{Cl}_{2}\right.$ )
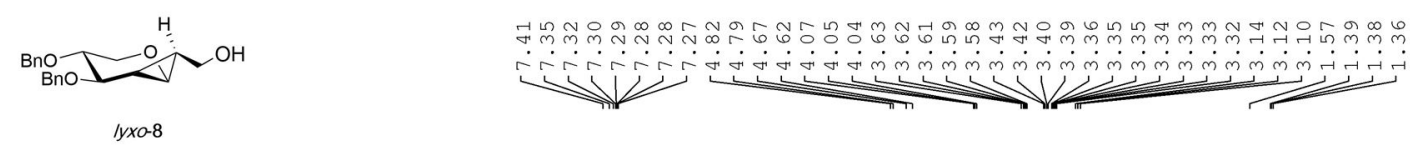

$1 y \times 0-8$

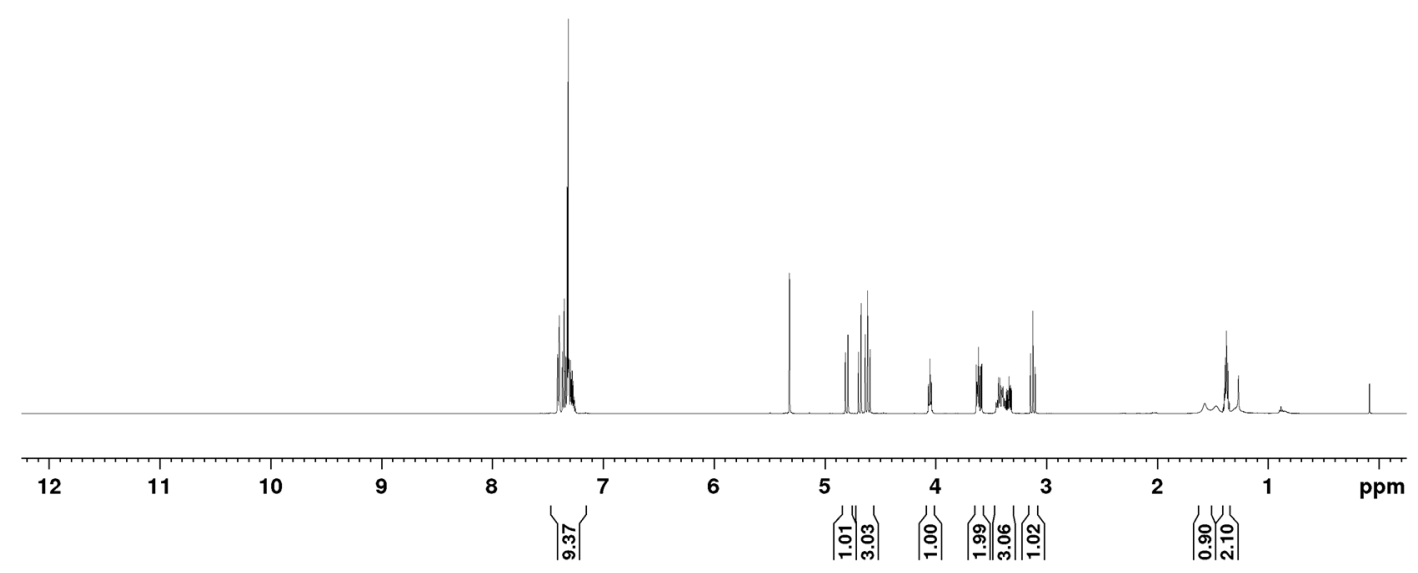

${ }^{13} \mathrm{C}$ NMR (125 MHz, $\mathrm{CD}_{2} \mathrm{Cl}_{2}$ )
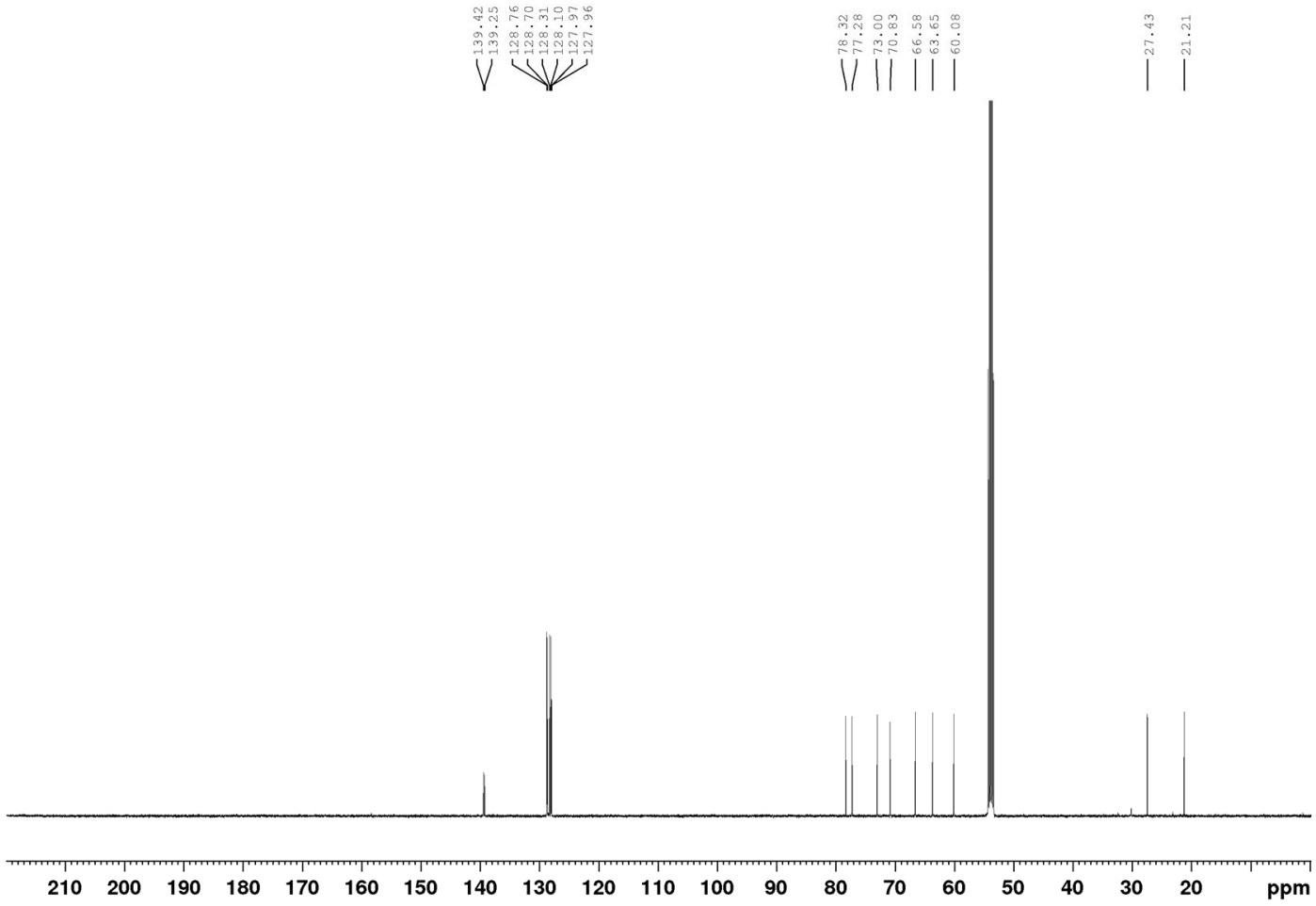
${ }^{1} \mathrm{H}$ NMR $\left(500 \mathrm{MHz}, \mathrm{CD}_{2} \mathrm{Cl}_{2}\right.$ )

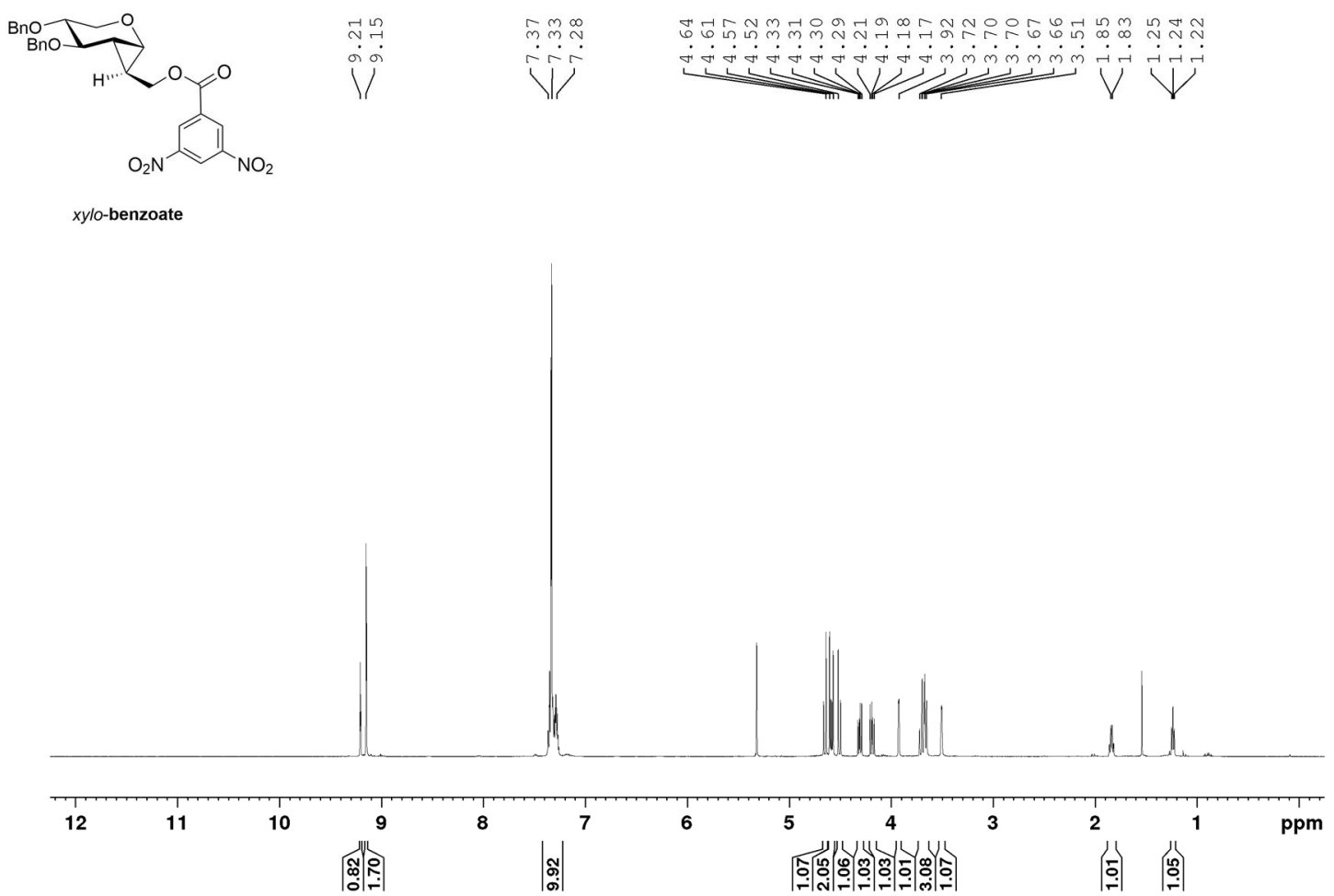

${ }^{13} \mathrm{C}$ NMR (125 MHz, $\mathrm{CD}_{2} \mathrm{Cl}_{2}$ )

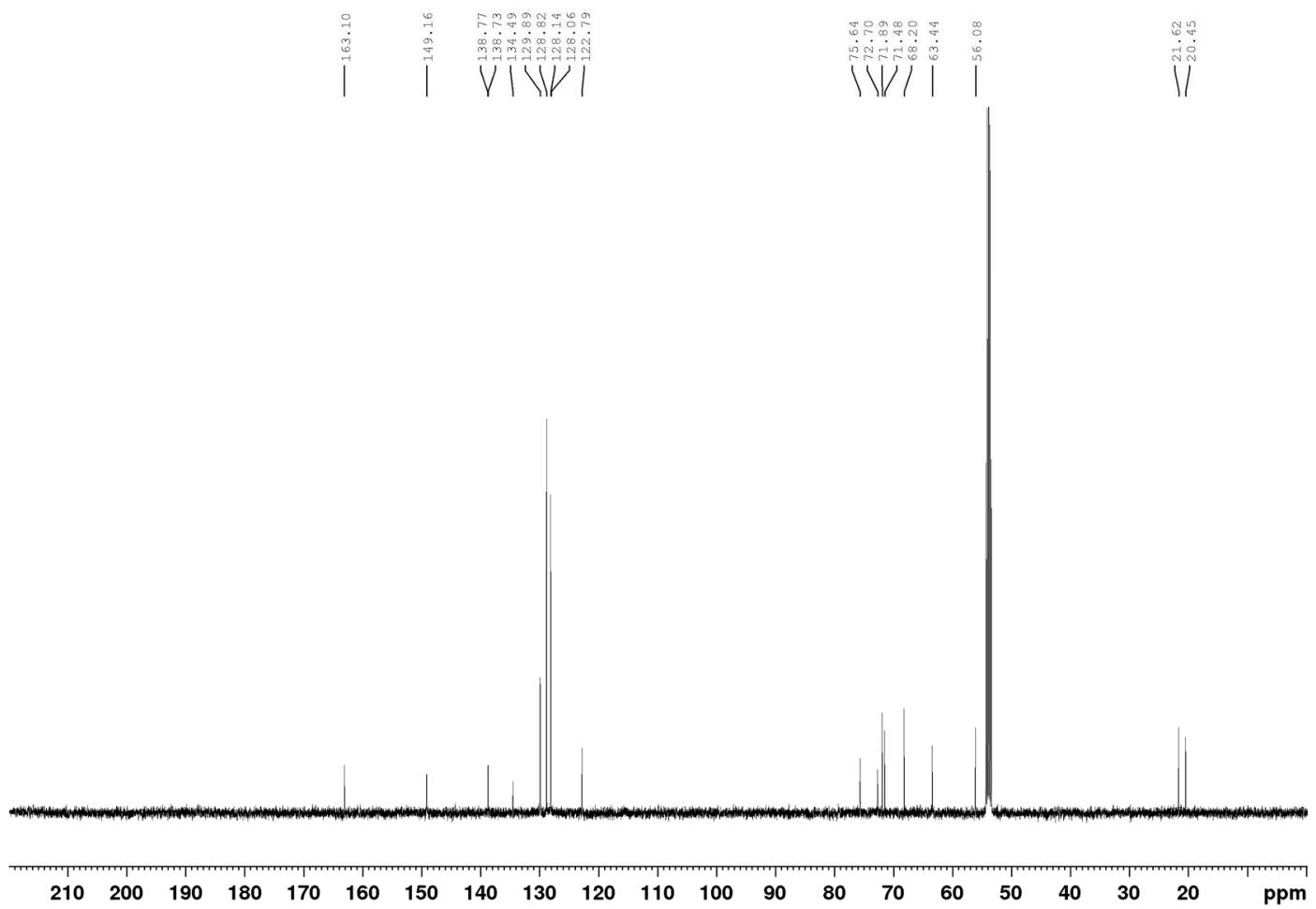


${ }^{1} \mathrm{H}$ NMR $\left(500 \mathrm{MHz}, \mathrm{CD}_{2} \mathrm{Cl}_{2}\right)$

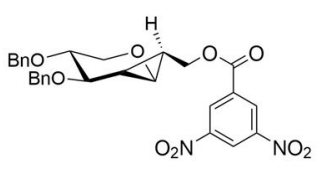

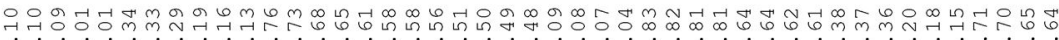

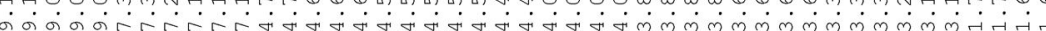

lyxa-benzoate

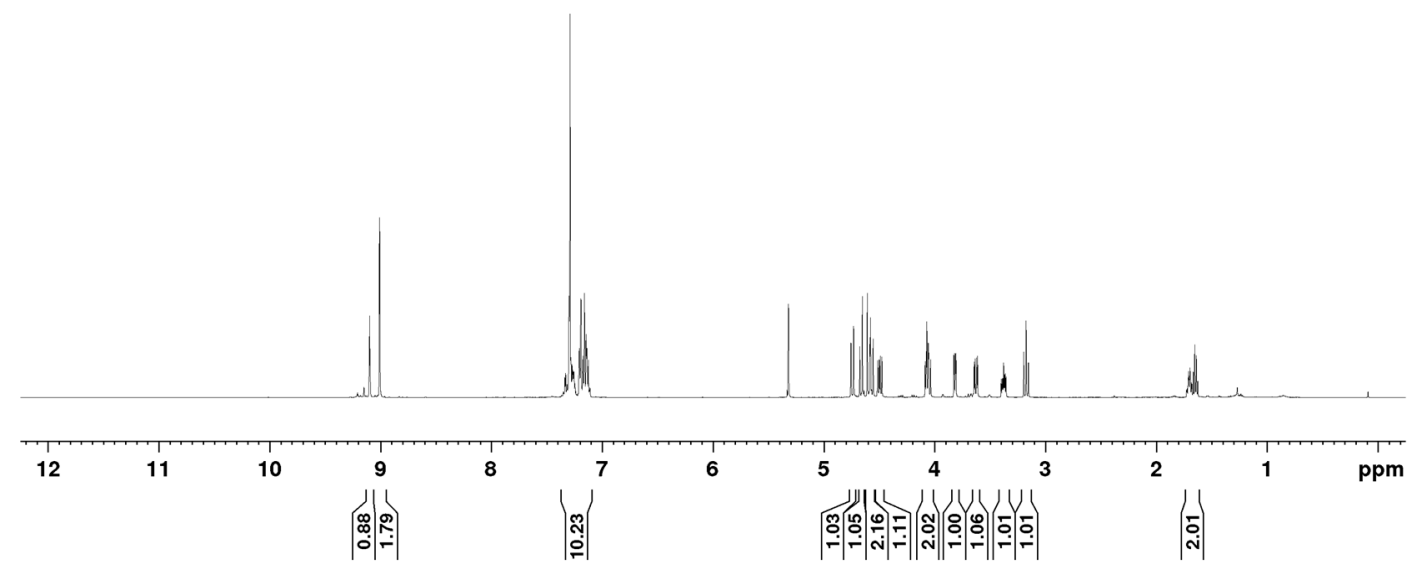

${ }^{13} \mathrm{C}$ NMR $\left(125 \mathrm{MHz}, \mathrm{CD}_{2} \mathrm{Cl}_{2}\right)$

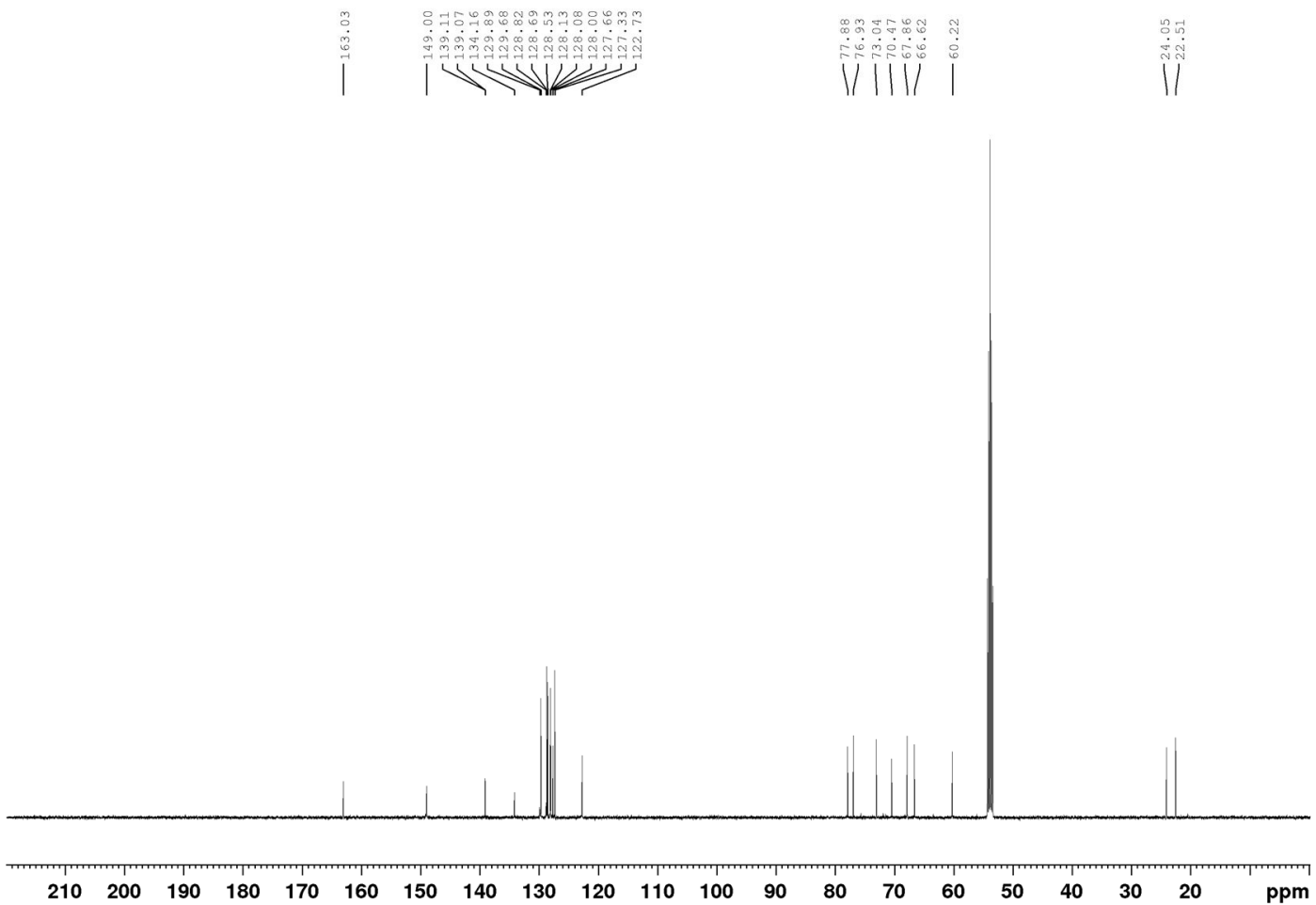




\section{8c. Xanthates 9}

${ }^{1} \mathrm{H}$ NMR $\left(600 \mathrm{MHz}, \mathrm{CD}_{2} \mathrm{Cl}_{2}\right)$

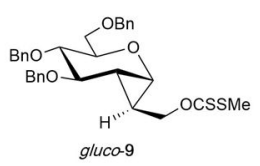

gluca-9

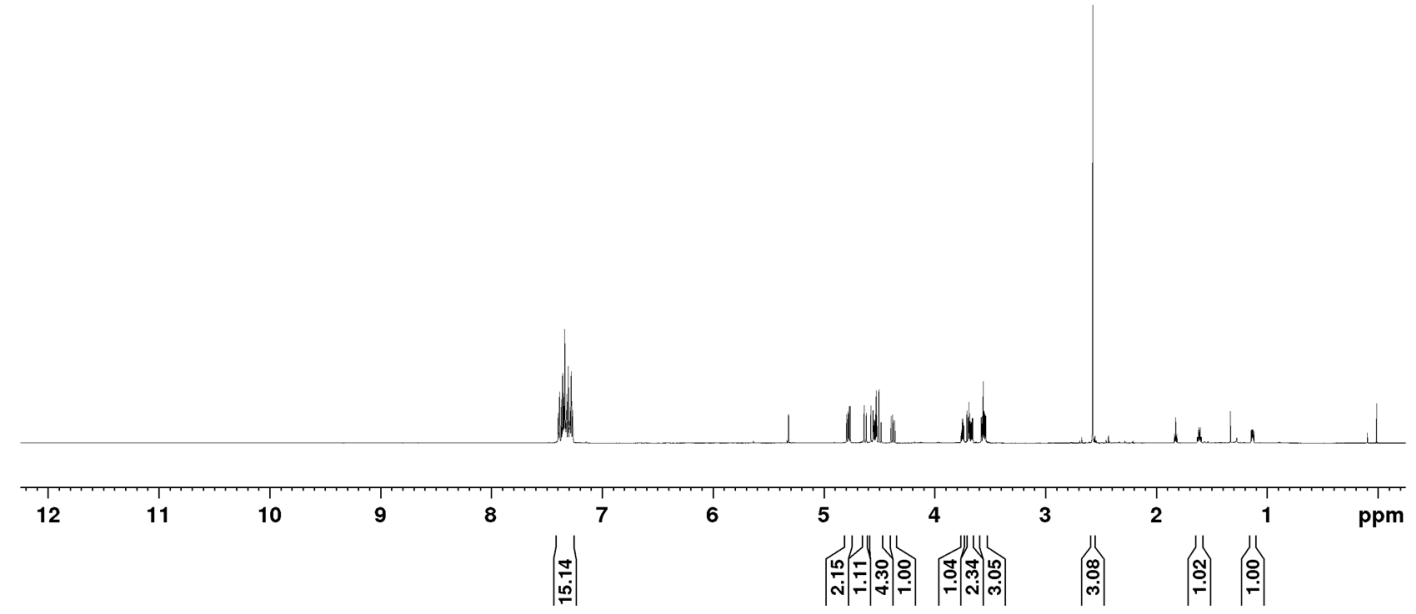

${ }^{13} \mathrm{C}$ NMR $\left(150 \mathrm{MHz}, \mathrm{CD}_{2} \mathrm{Cl}_{2}\right)$
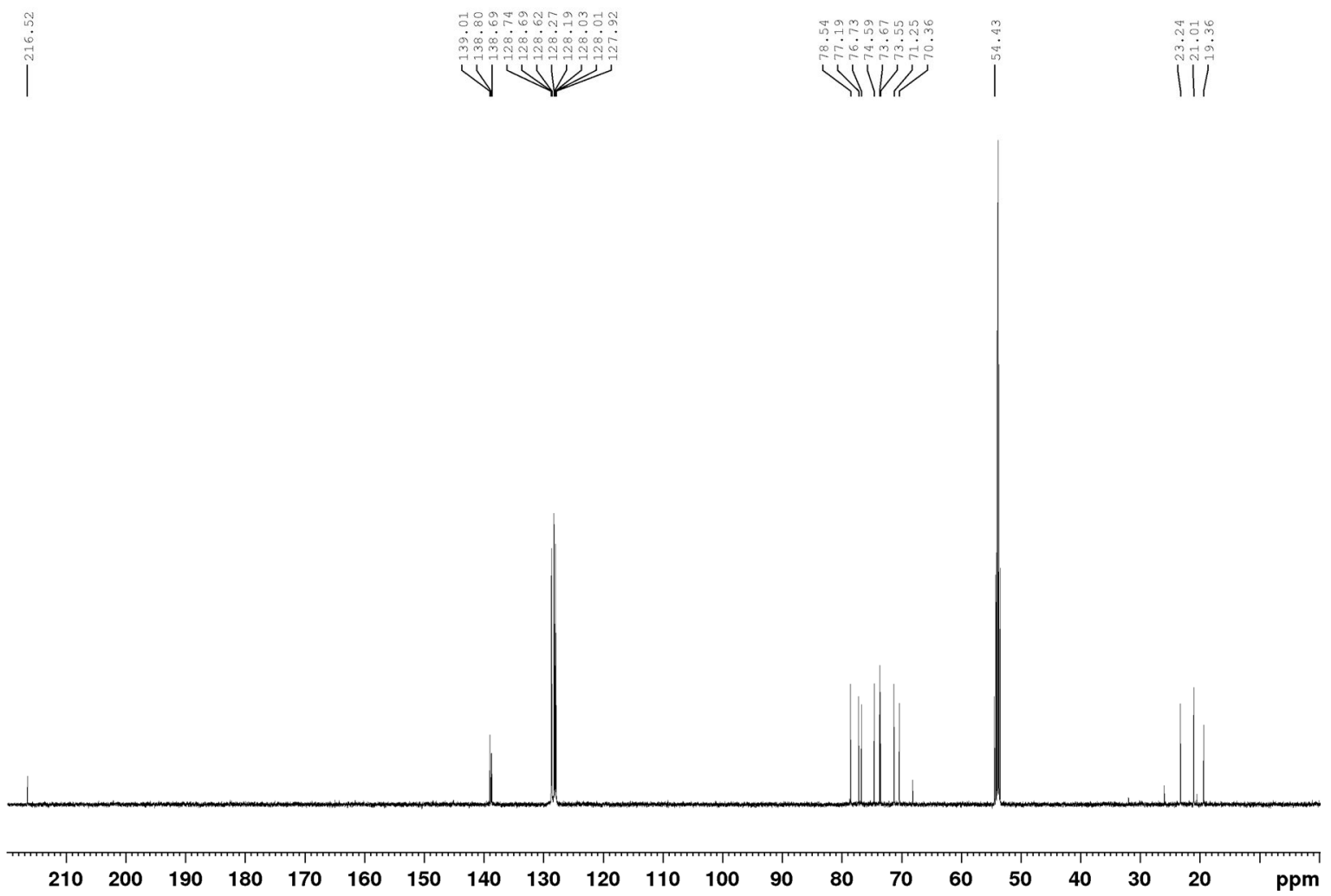
${ }^{1} \mathrm{H}$ NMR $\left(600 \mathrm{MHz}, \mathrm{CD}_{2} \mathrm{Cl}_{2}\right)$

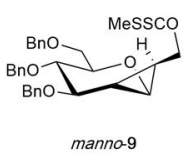

manna-9

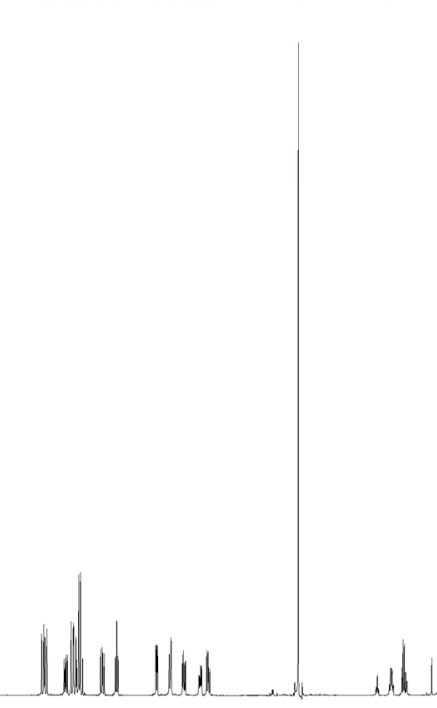

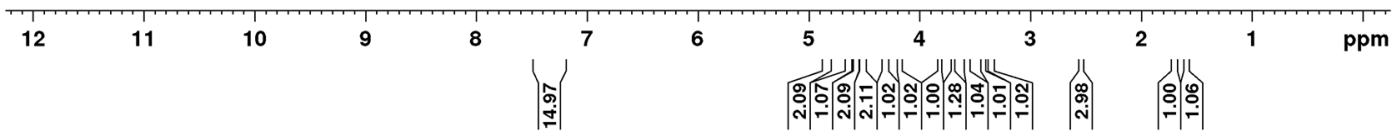

${ }^{13} \mathrm{C}$ NMR $\left(150 \mathrm{MHz}, \mathrm{CD}_{2} \mathrm{Cl}_{2}\right)$
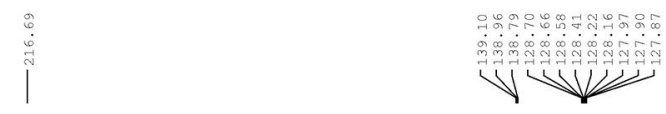

v|

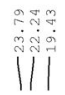

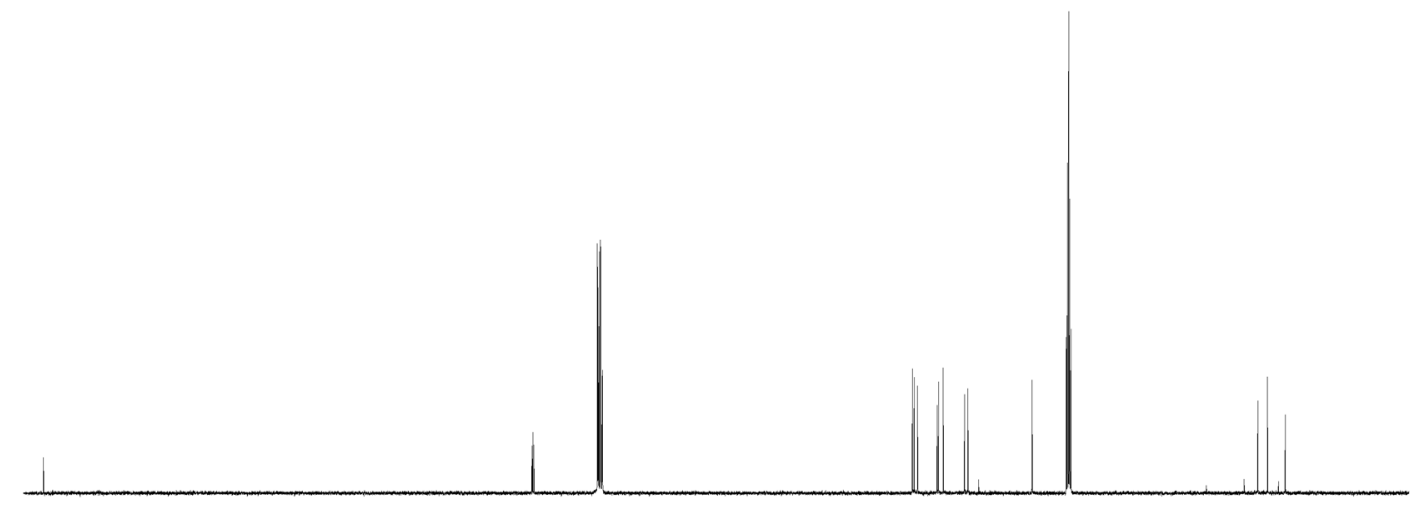

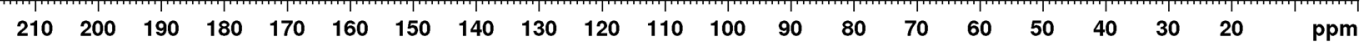


${ }^{1} \mathrm{H}$ NMR $\left(500 \mathrm{MHz}, \mathrm{CD}_{2} \mathrm{Cl}_{2}\right)$
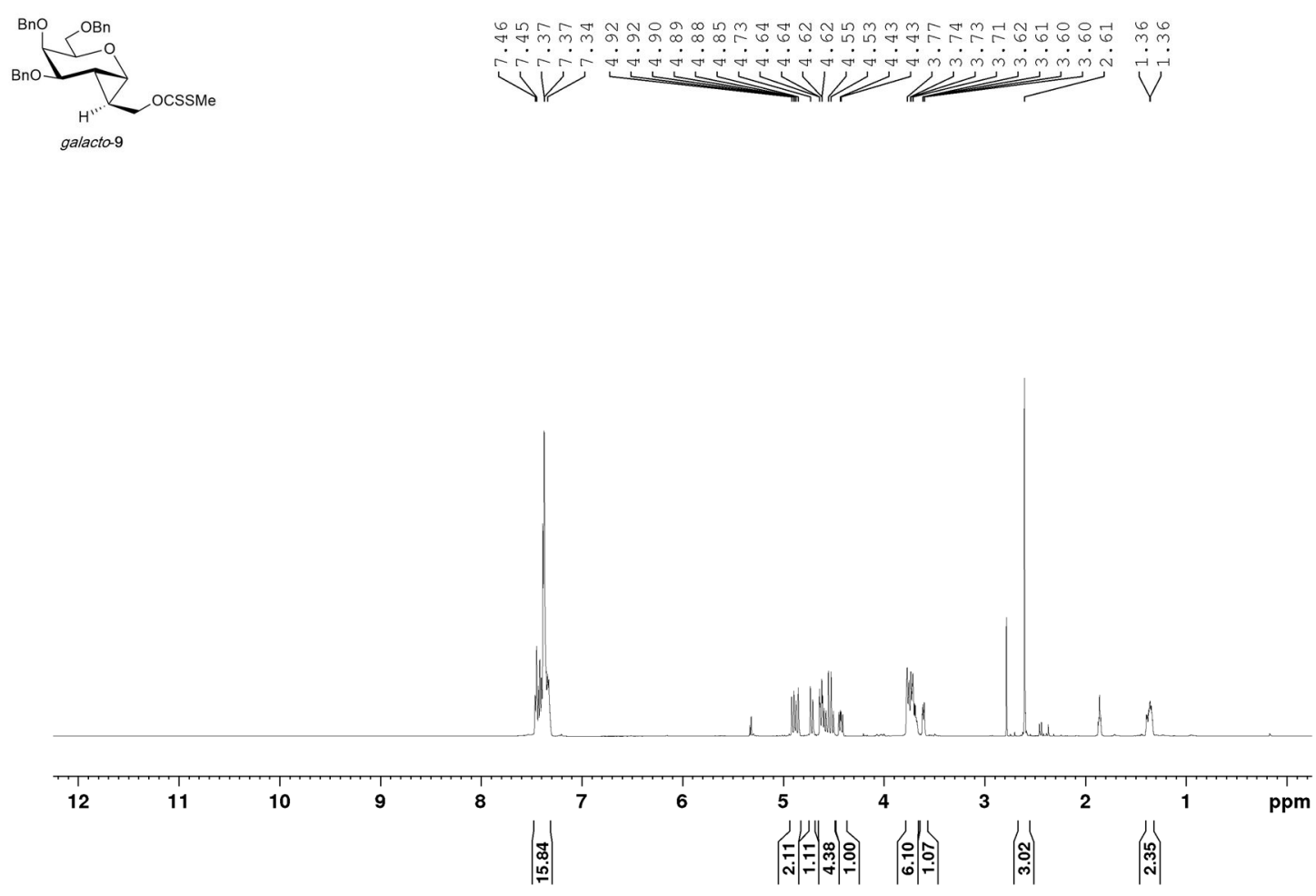

${ }^{13} \mathrm{C}$ NMR (125 MHz, $\mathrm{CD}_{2} \mathrm{Cl}_{2}$ )
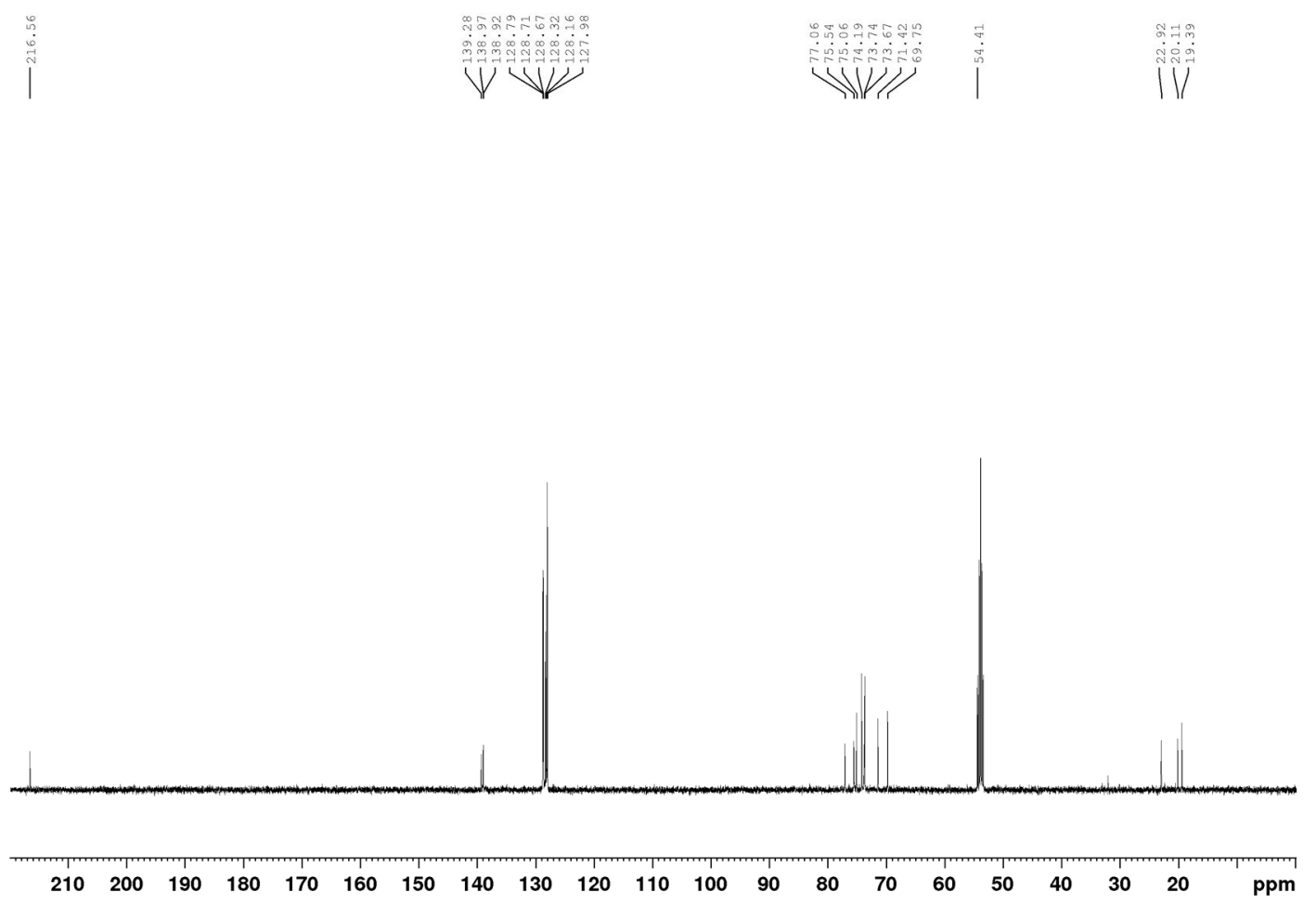

S43 
${ }^{1} \mathrm{H}$ NMR $\left(500 \mathrm{MHz}, \mathrm{CD}_{2} \mathrm{Cl}_{2}\right.$ )
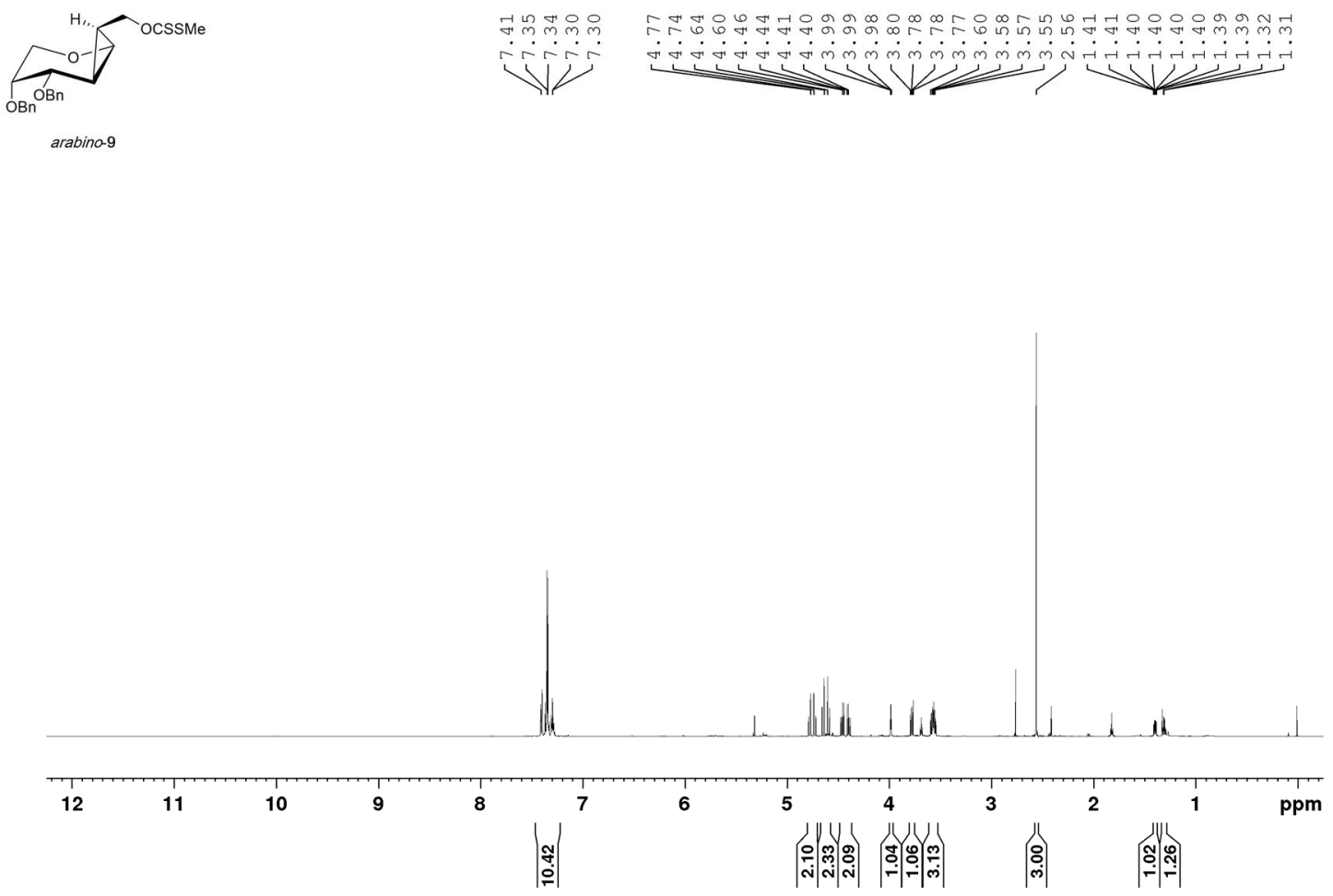

${ }^{13} \mathrm{C}$ NMR $\left(125 \mathrm{MHz}, \mathrm{CD}_{2} \mathrm{Cl}_{2}\right)$
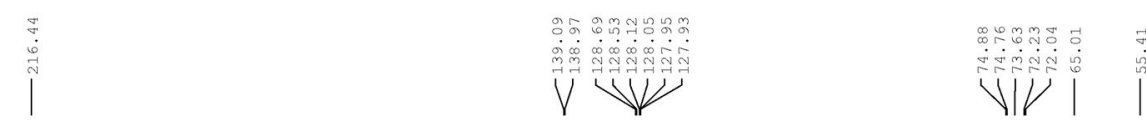

ฟู่

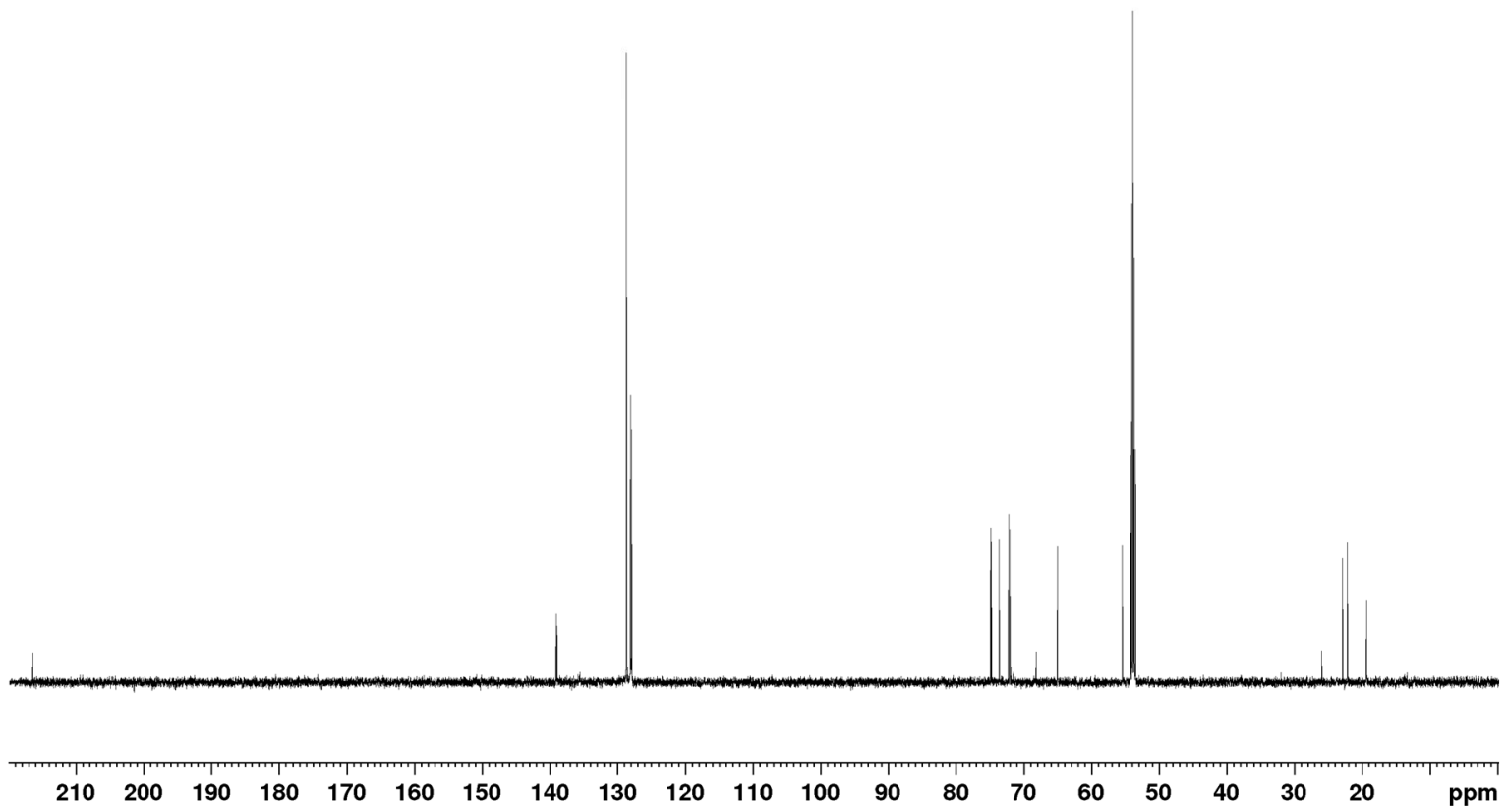


${ }^{1} \mathrm{H}$ NMR $\left(500 \mathrm{MHz}, \mathrm{CD}_{2} \mathrm{Cl}_{2}\right)$

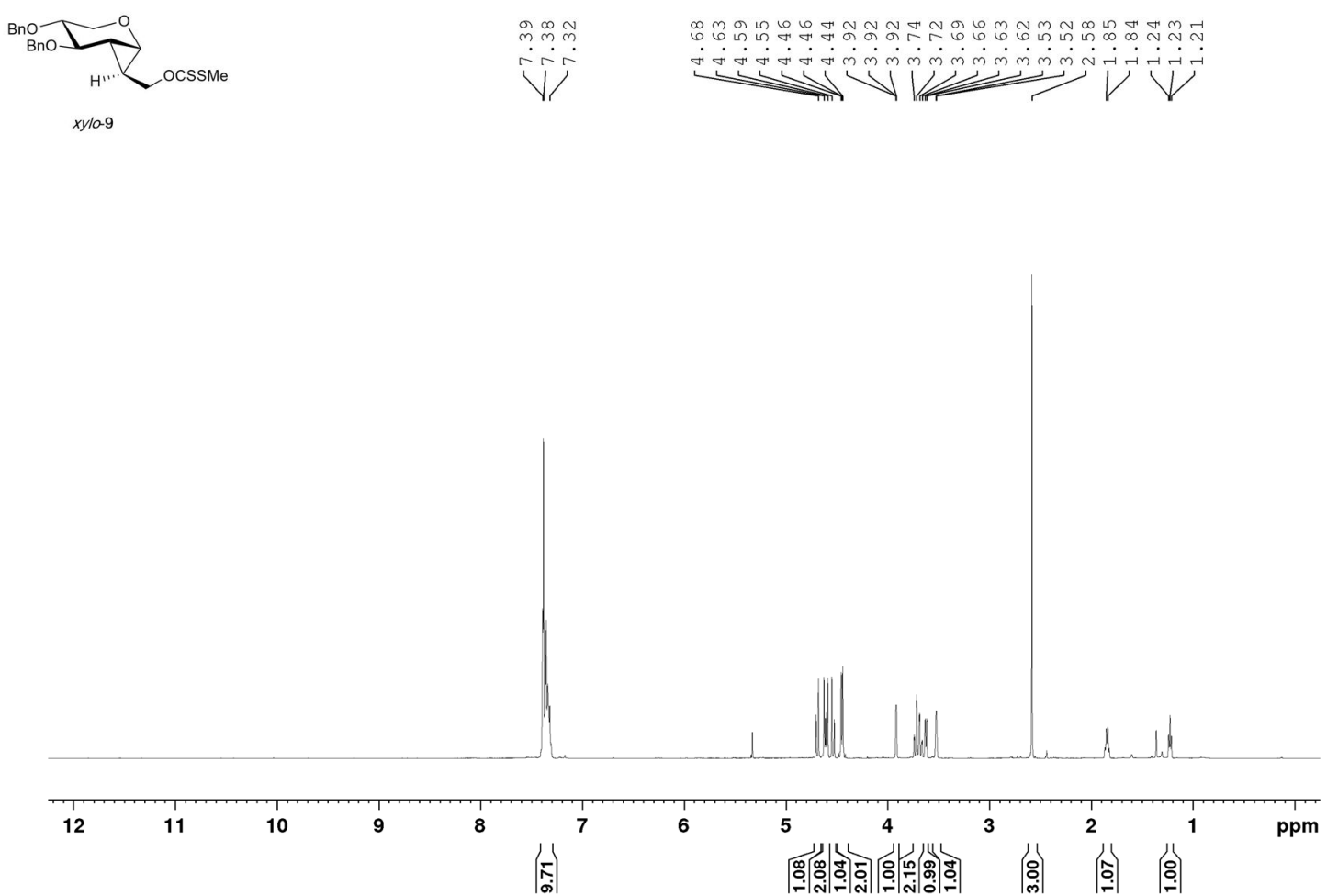

${ }^{13} \mathrm{C}$ NMR (125 MHz, $\mathrm{CD}_{2} \mathrm{Cl}_{2}$ )

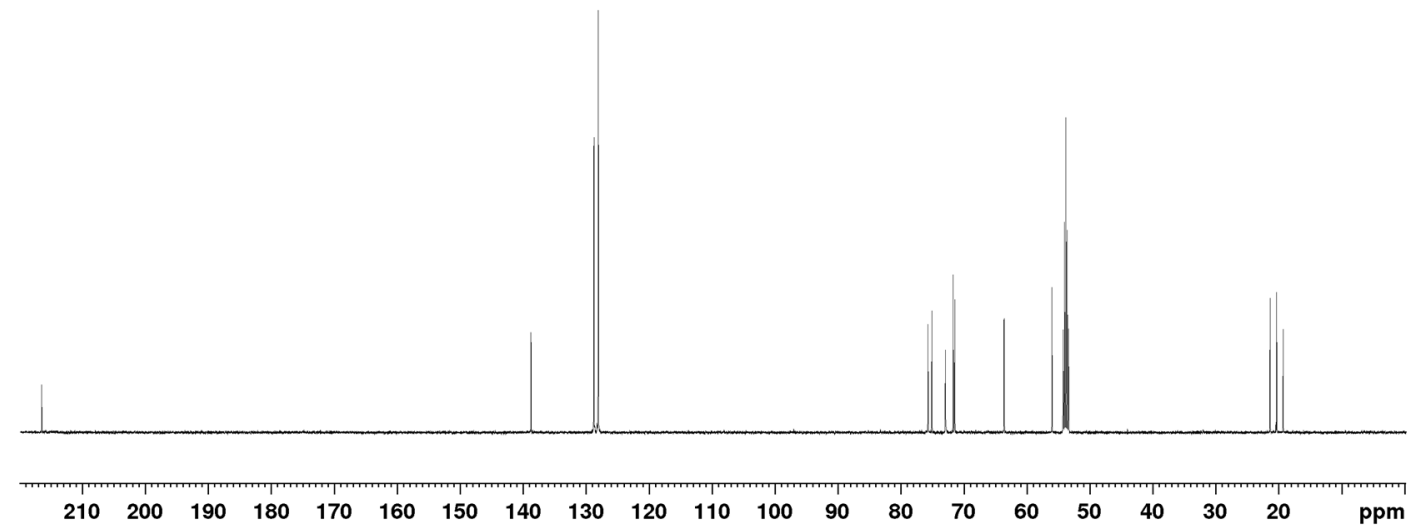




\section{8d. Products of radical reactions 5}

${ }^{1} \mathrm{H}$ NMR $\left(500 \mathrm{MHz}, \mathrm{CD}_{2} \mathrm{Cl}_{2}\right)$
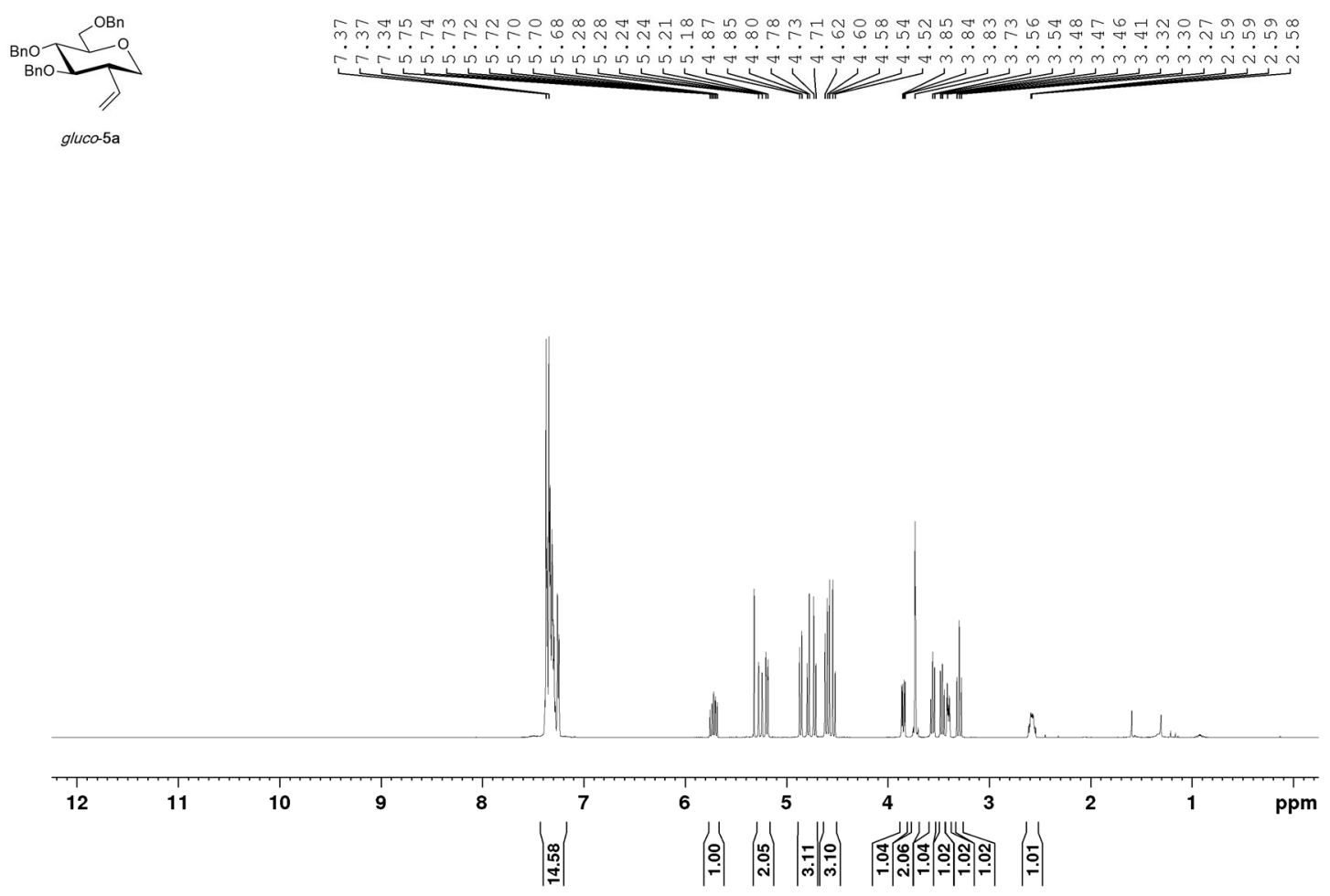

${ }^{13} \mathrm{C}$ NMR $\left(125 \mathrm{MHz}, \mathrm{CD}_{2} \mathrm{Cl}_{2}\right)$

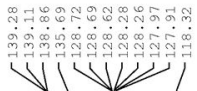

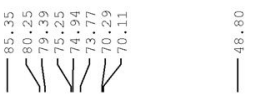

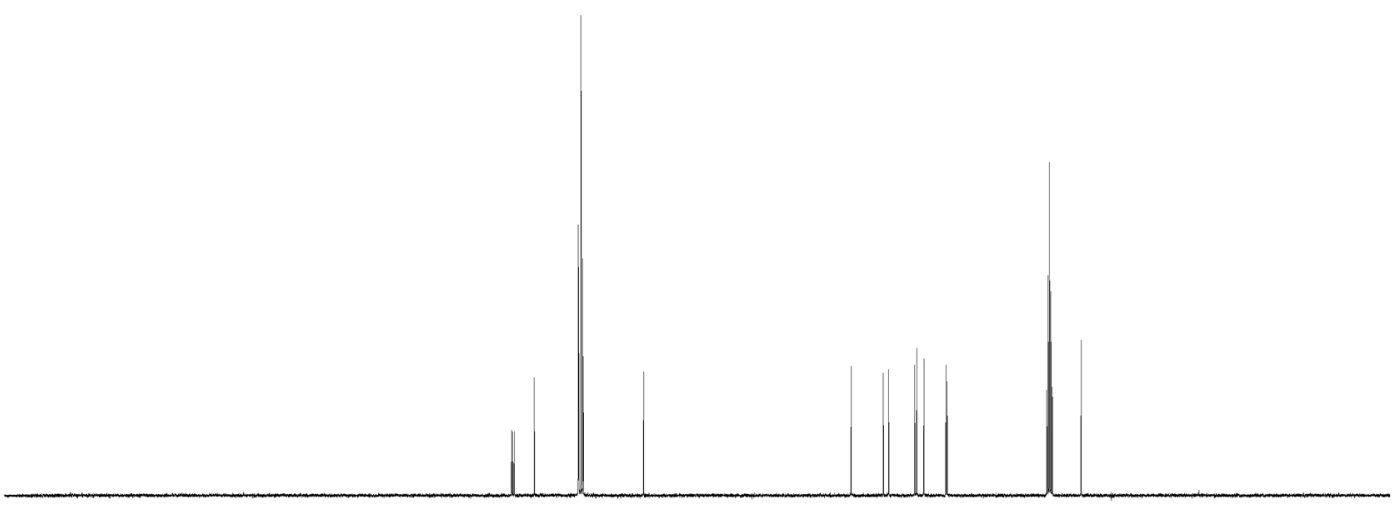

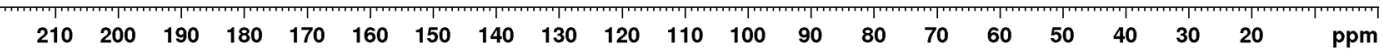


${ }^{1} \mathrm{H} \operatorname{NMR}\left(500 \mathrm{MHz}, \mathrm{CD}_{2} \mathrm{Cl}_{2}\right)$

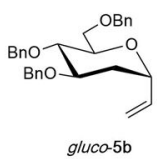

gluco-5b

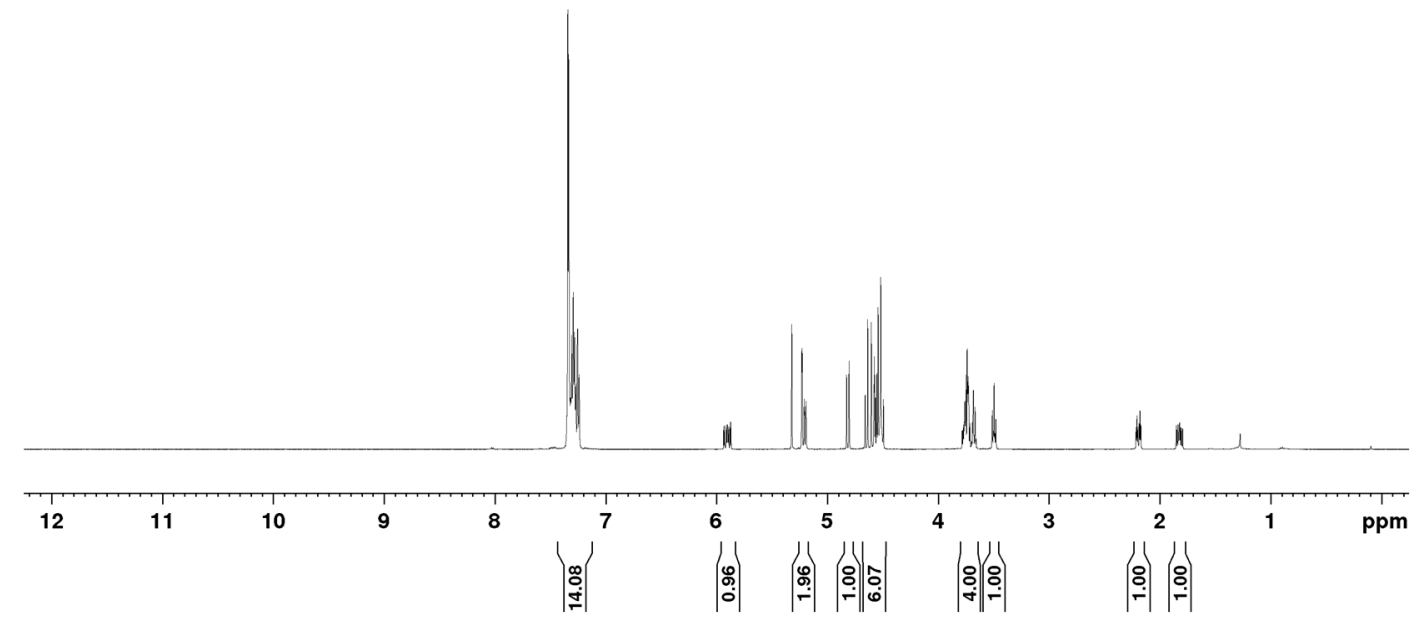

${ }^{13} \mathrm{C}$ NMR $\left(125 \mathrm{MHz}, \mathrm{CD}_{2} \mathrm{Cl}_{2}\right)$
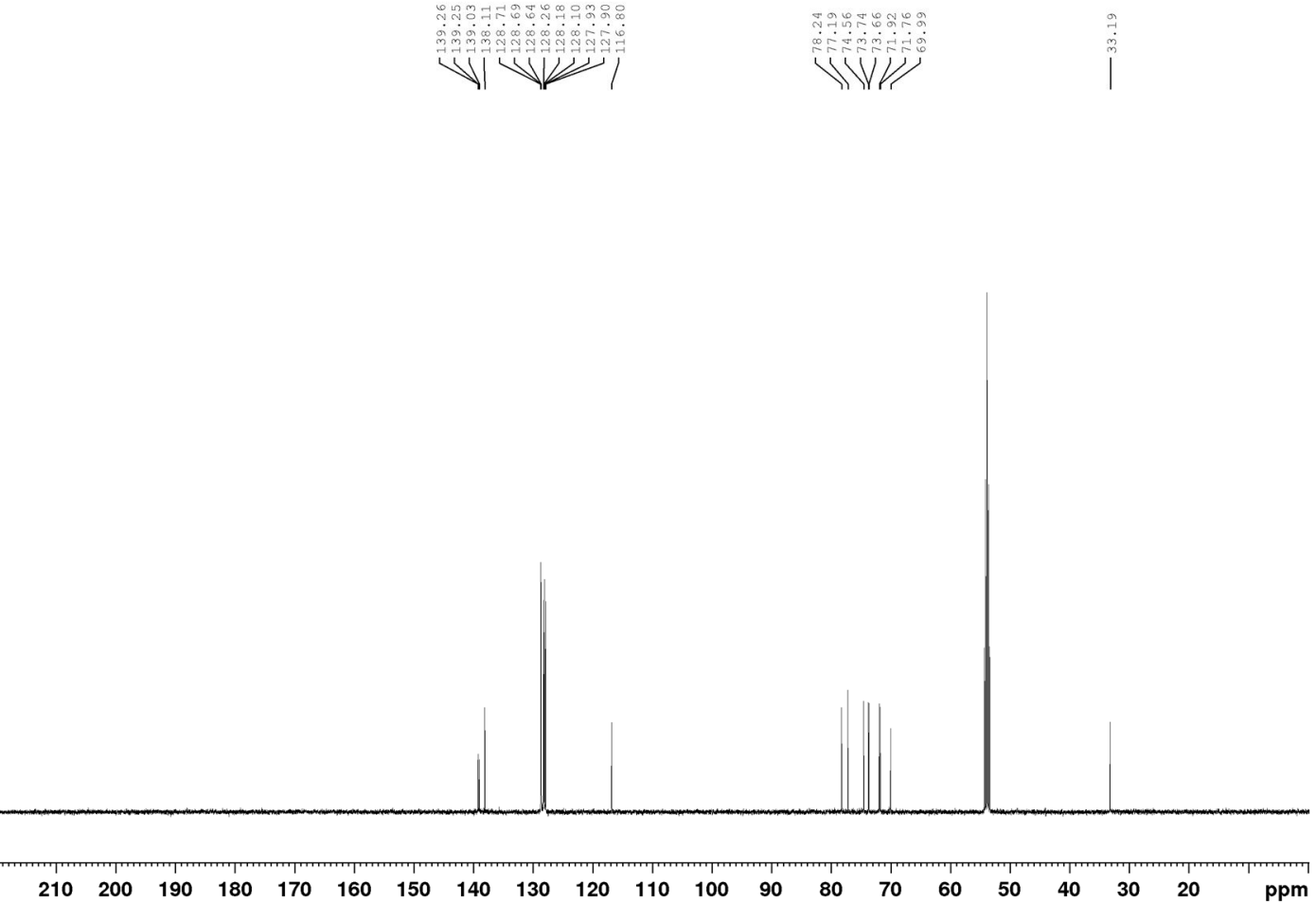
${ }^{1} \mathrm{H}$ NMR $\left(500 \mathrm{MHz}, \mathrm{CD}_{2} \mathrm{Cl}_{2}\right)$

$$
\text { manna-5a }
$$

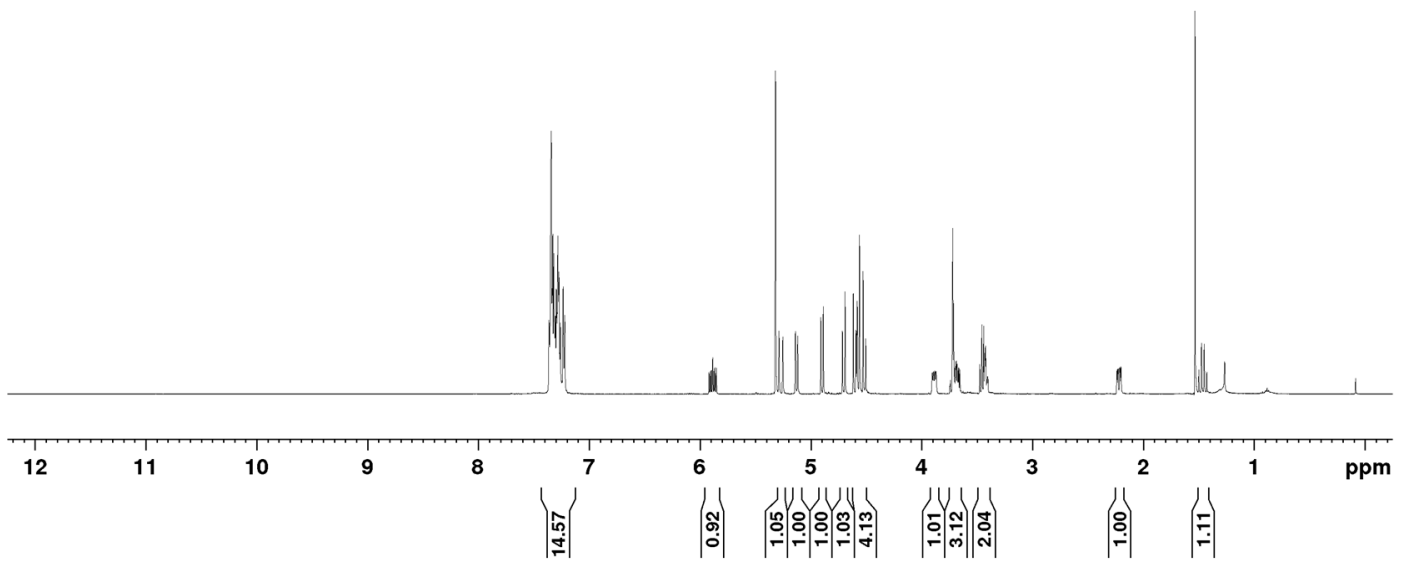

${ }^{13} \mathrm{C}$ NMR $\left(125 \mathrm{MHz}, \mathrm{CD}_{2} \mathrm{Cl}_{2}\right)$
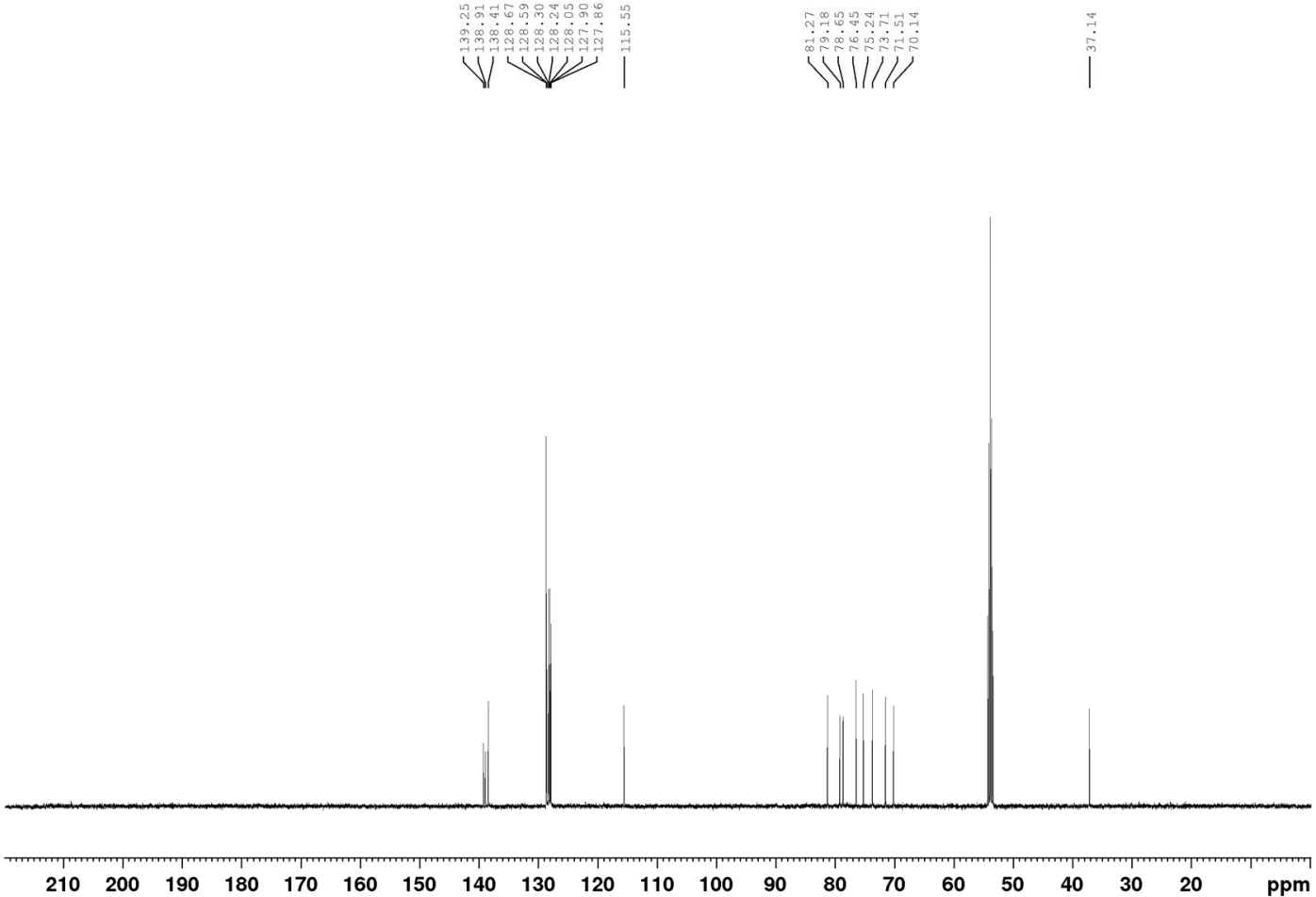
${ }^{1} \mathrm{H}$ NMR $\left(500 \mathrm{MHz}, \mathrm{CD}_{2} \mathrm{Cl}_{2}\right)$

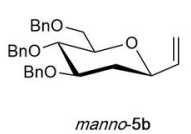

manno-5b

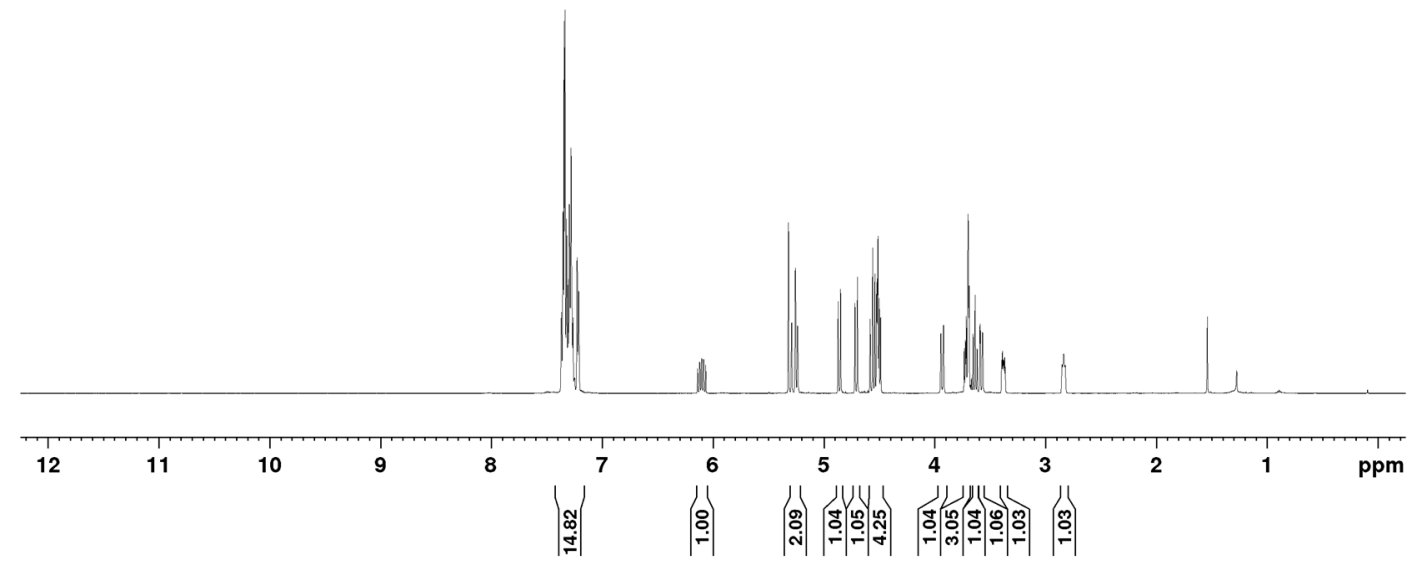

${ }^{13} \mathrm{C}$ NMR $\left(125 \mathrm{MHz}, \mathrm{CD}_{2} \mathrm{Cl}_{2}\right)$

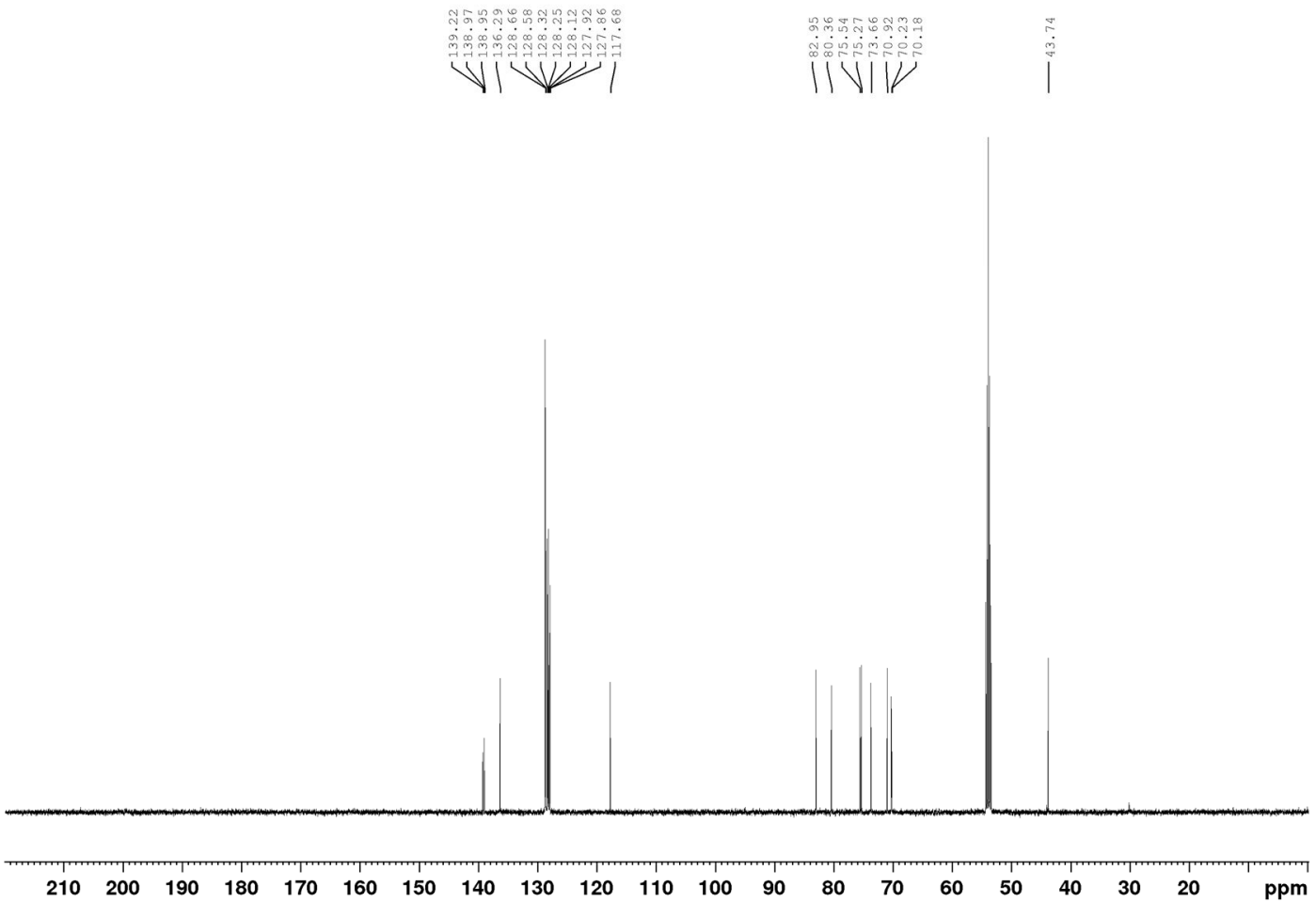


${ }^{1} \mathrm{H}$ NMR $\left(600 \mathrm{MHz}, \mathrm{CD}_{2} \mathrm{Cl}_{2}\right)$
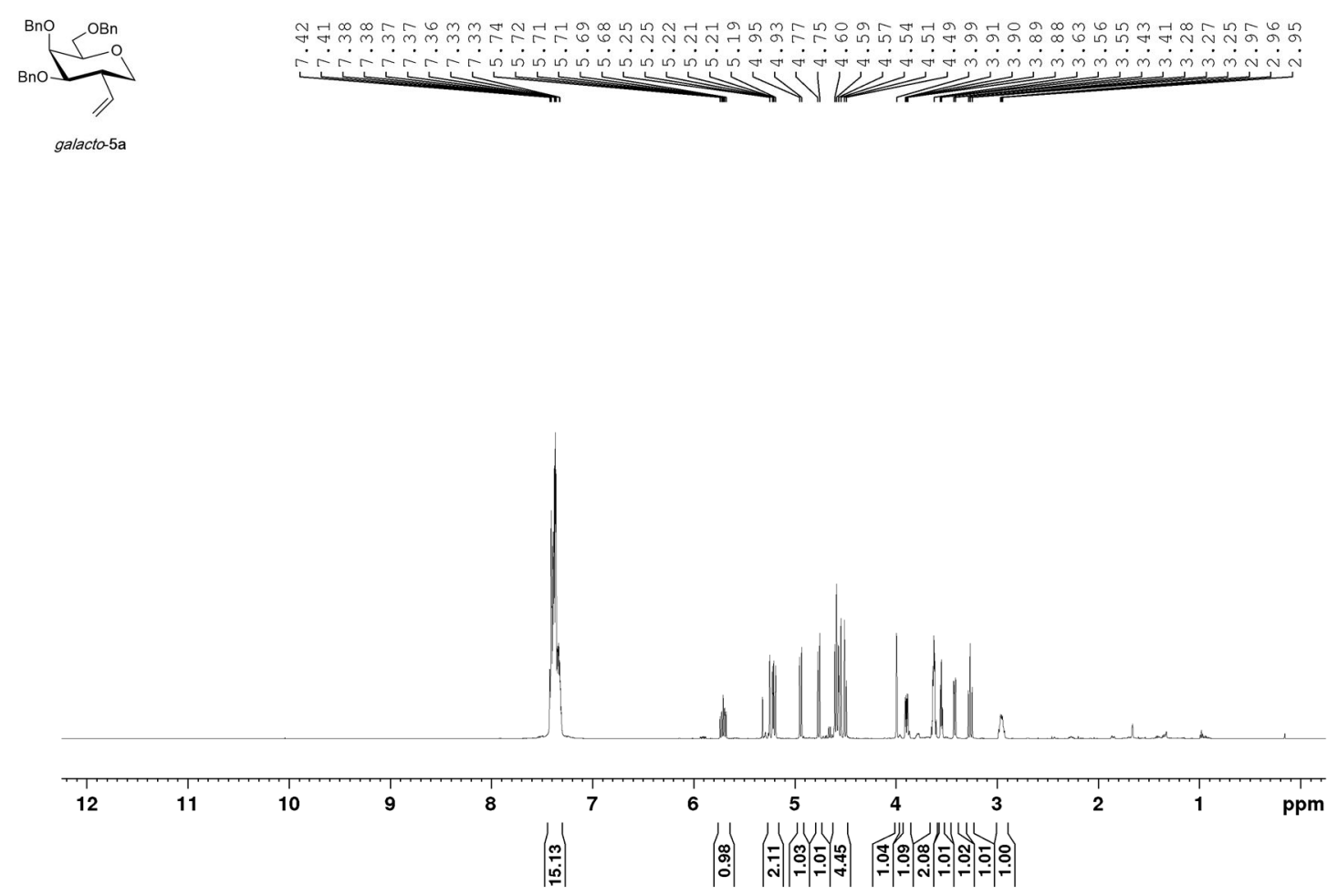

${ }^{13} \mathrm{C}$ NMR $\left(150 \mathrm{MHz}, \mathrm{CD}_{2} \mathrm{Cl}_{2}\right)$

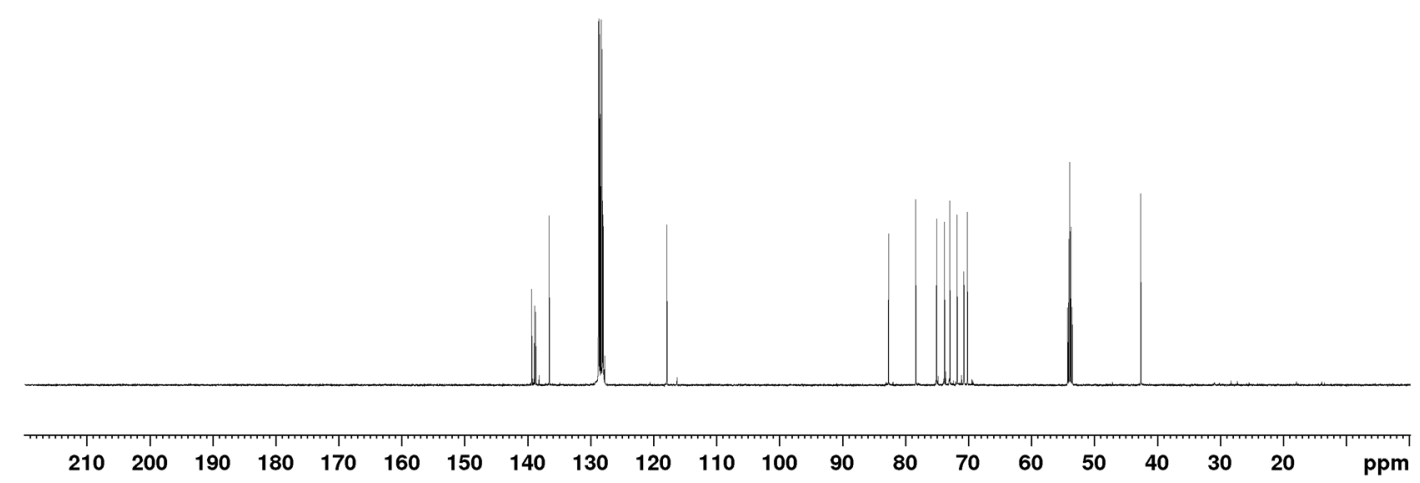


${ }^{1} \mathrm{H}$ NMR (600 MHz, $\mathrm{CD}_{2} \mathrm{Cl}_{2}$ )
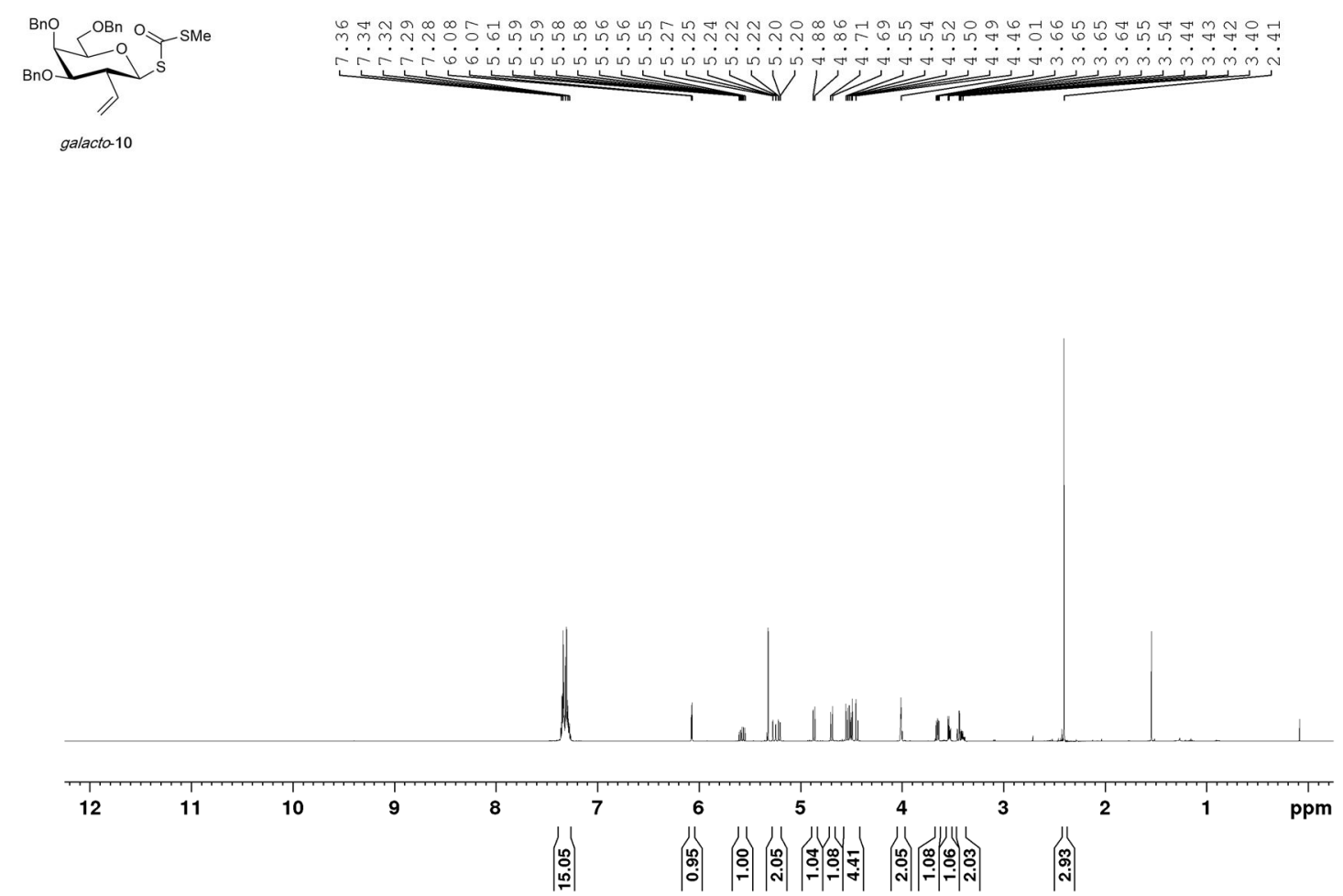

${ }^{13} \mathrm{C}$ NMR (125 MHz, $\mathrm{CD}_{2} \mathrm{Cl}_{2}$ )

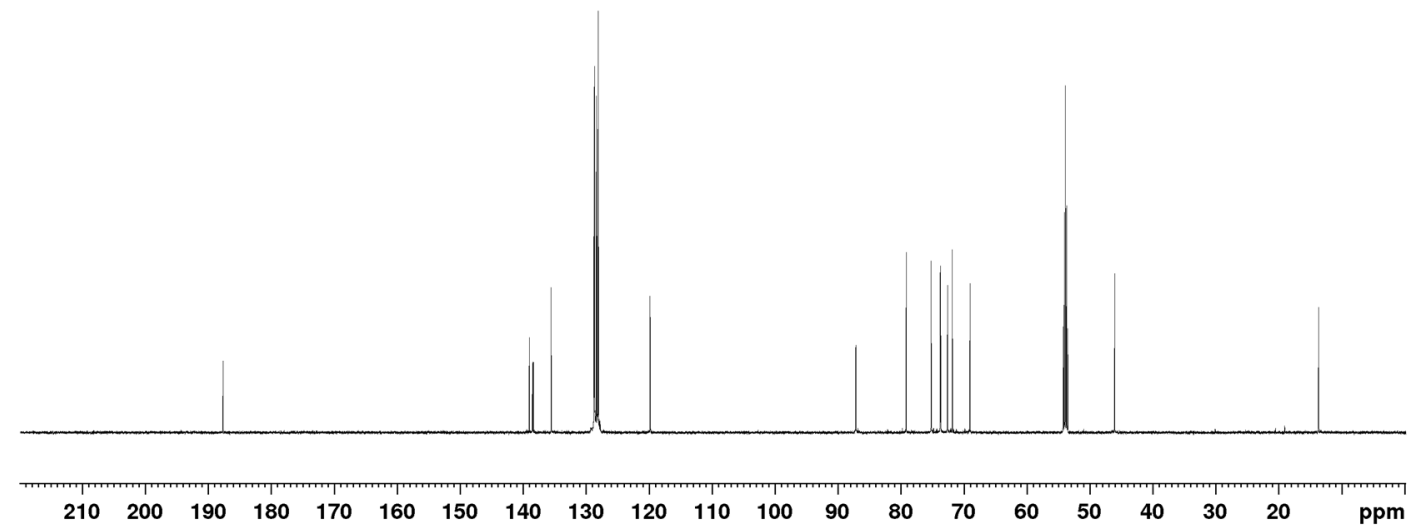


${ }^{1} \mathrm{H}$ NMR (600 MHz, $\mathrm{CD}_{2} \mathrm{Cl}_{2}$ )
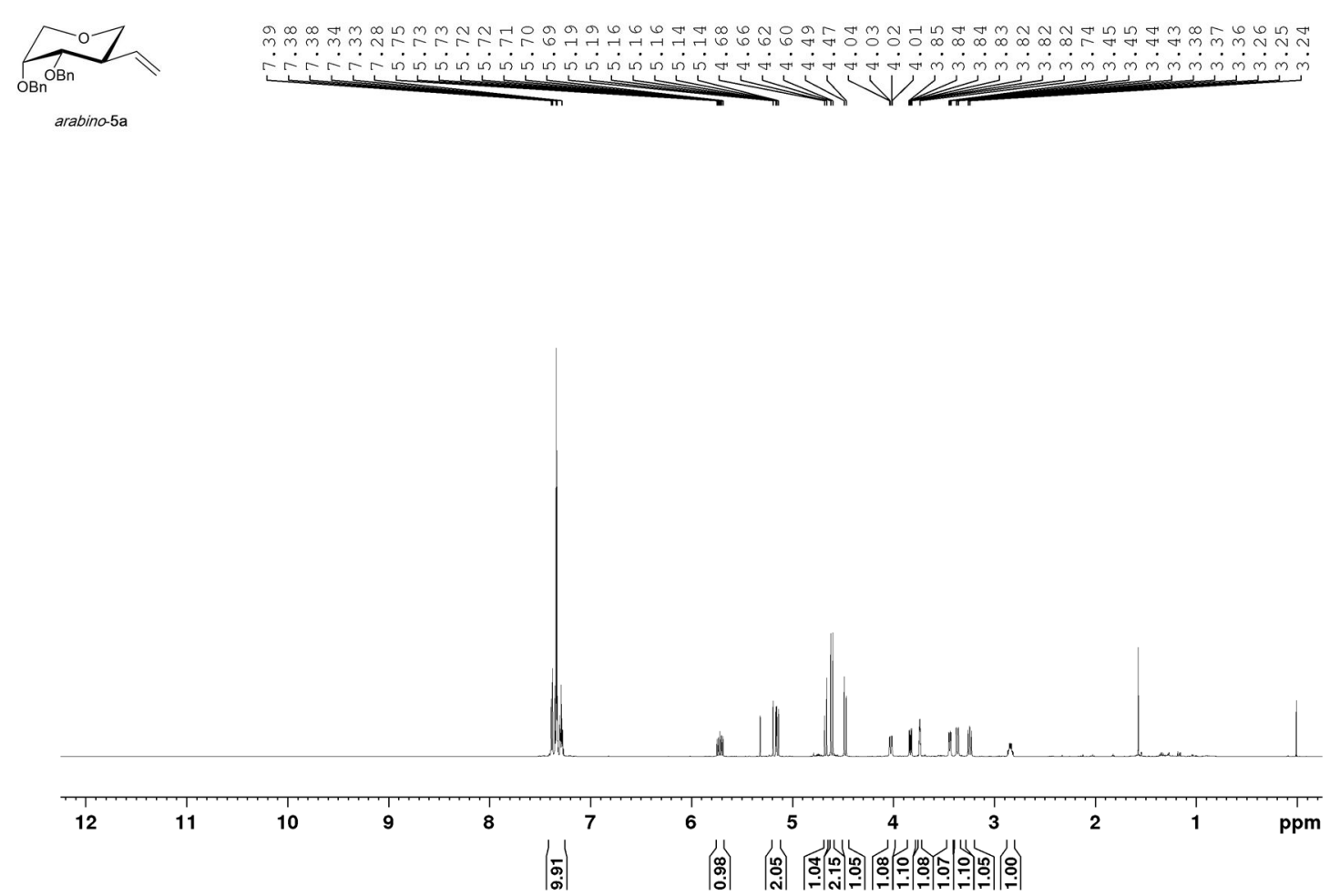

${ }^{13} \mathrm{C}$ NMR (125 MHz, $\mathrm{CD}_{2} \mathrm{Cl}_{2}$ )

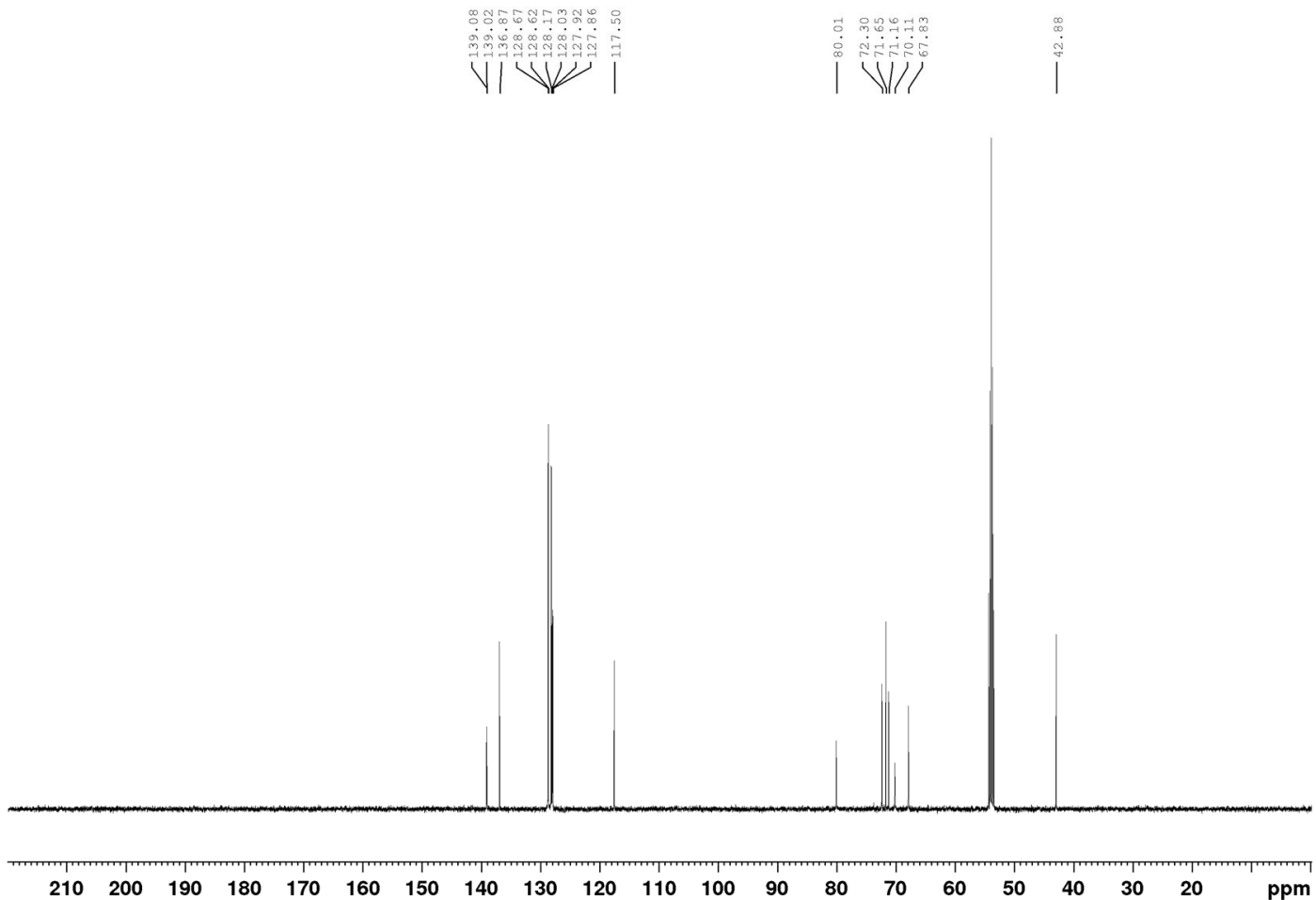


${ }^{1} \mathrm{H} \mathrm{NMR}\left(500 \mathrm{MHz}, \mathrm{CD}_{2} \mathrm{Cl}_{2}\right)$
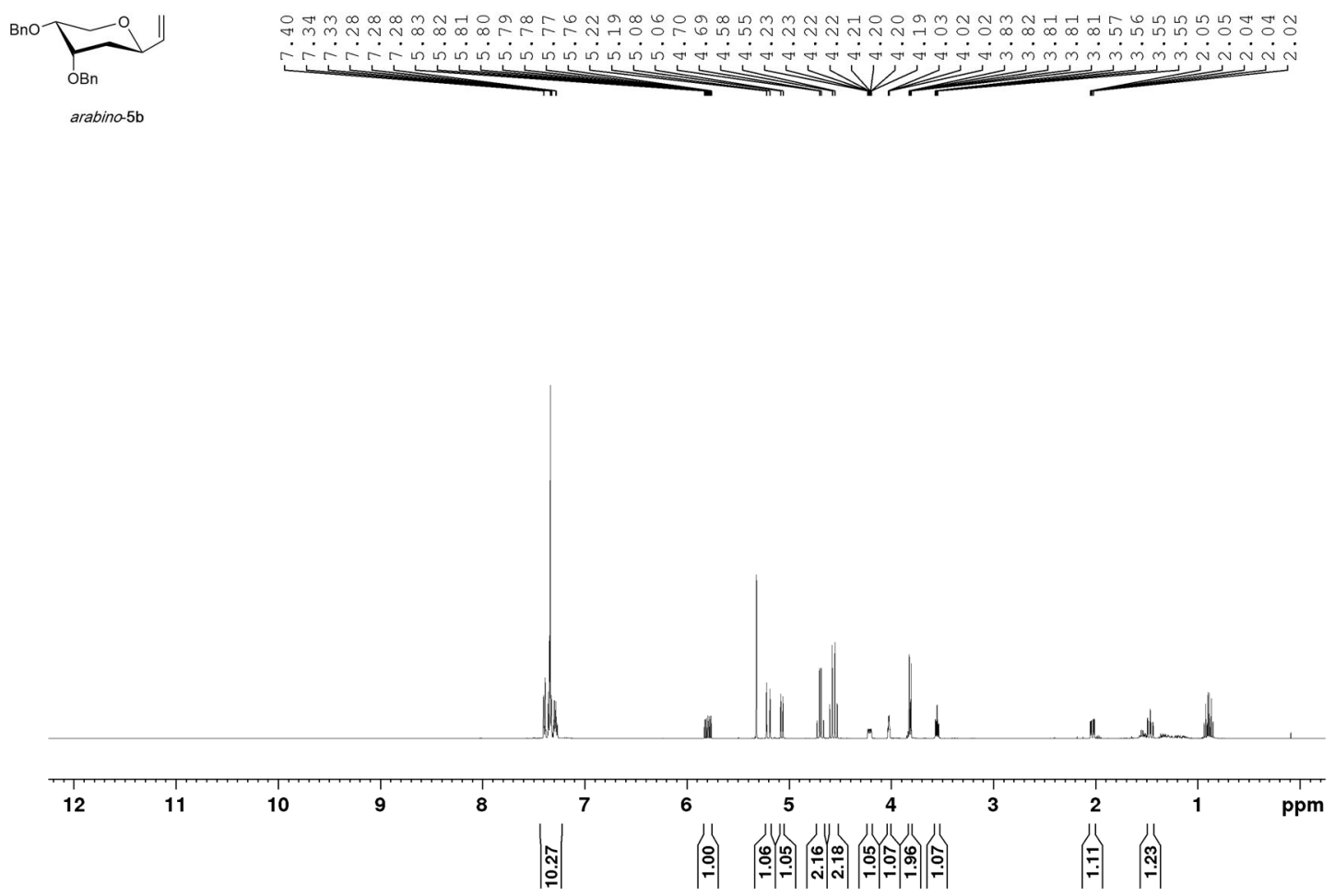

${ }^{13} \mathrm{C}$ NMR $\left(125 \mathrm{MHz}, \mathrm{CD}_{2} \mathrm{Cl}_{2}\right)$
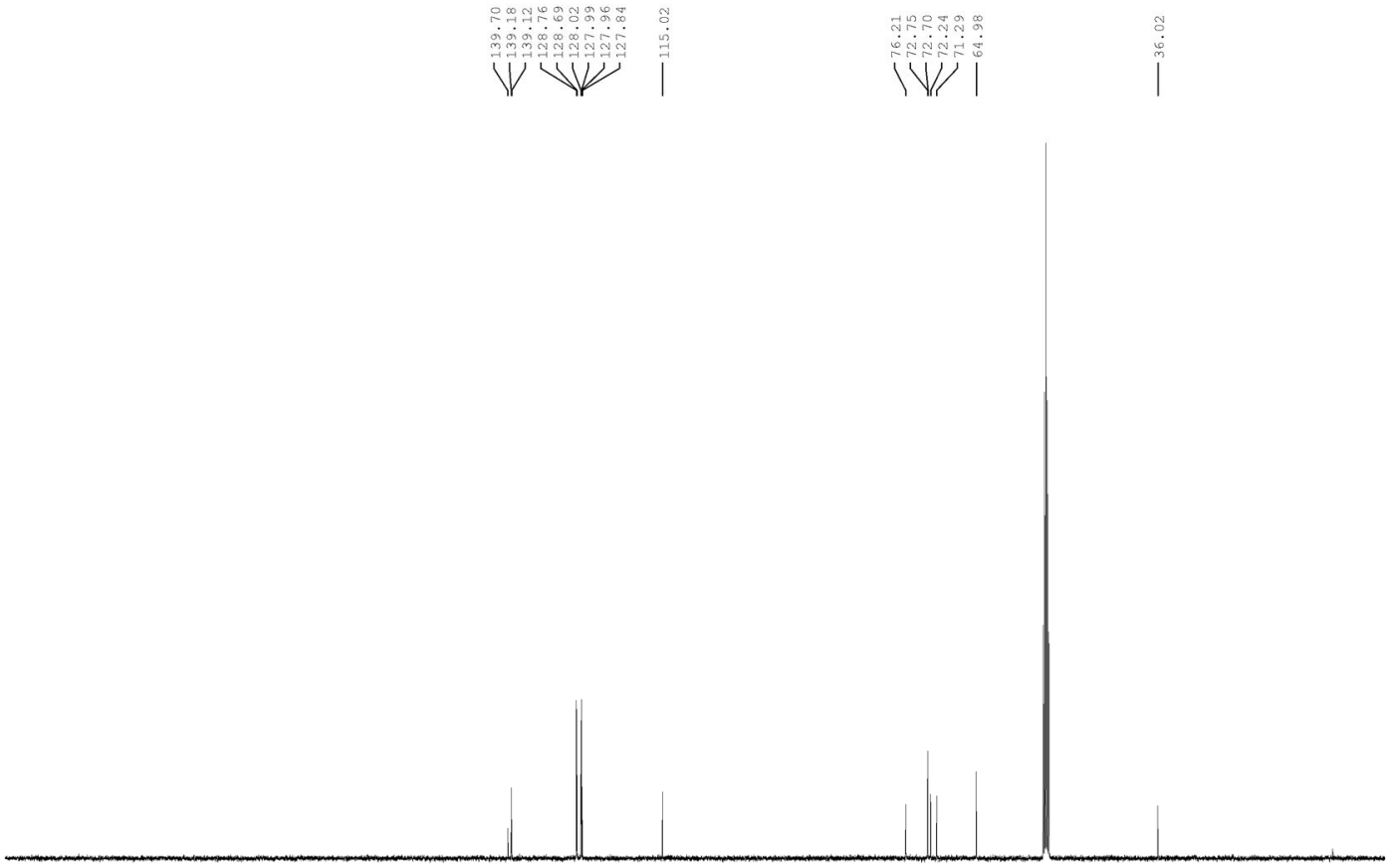

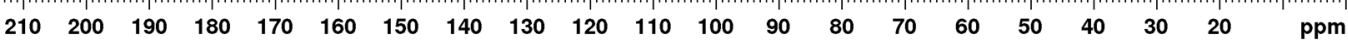


${ }^{1} \mathrm{H}$ NMR $\left(500 \mathrm{MHz}, \mathrm{CD}_{2} \mathrm{Cl}_{2}\right)$

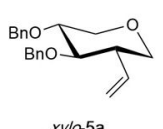

$x y / 0-5 a$

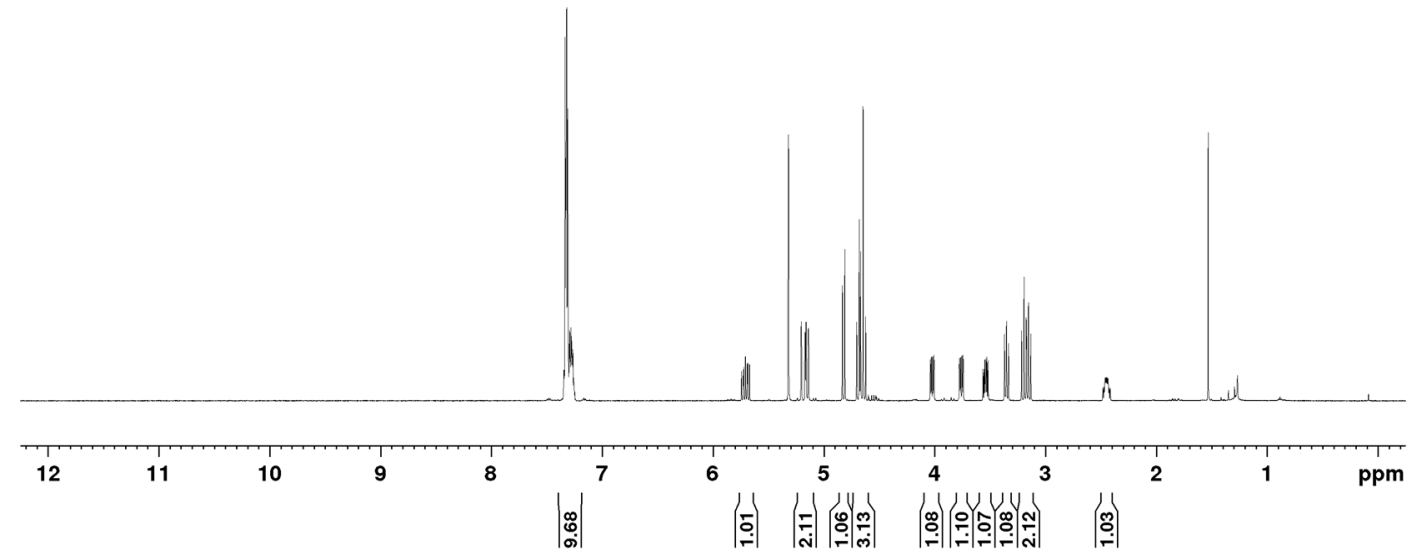

${ }^{13} \mathrm{C}$ NMR $\left(125 \mathrm{MHz}, \mathrm{CD}_{2} \mathrm{Cl}_{2}\right)$
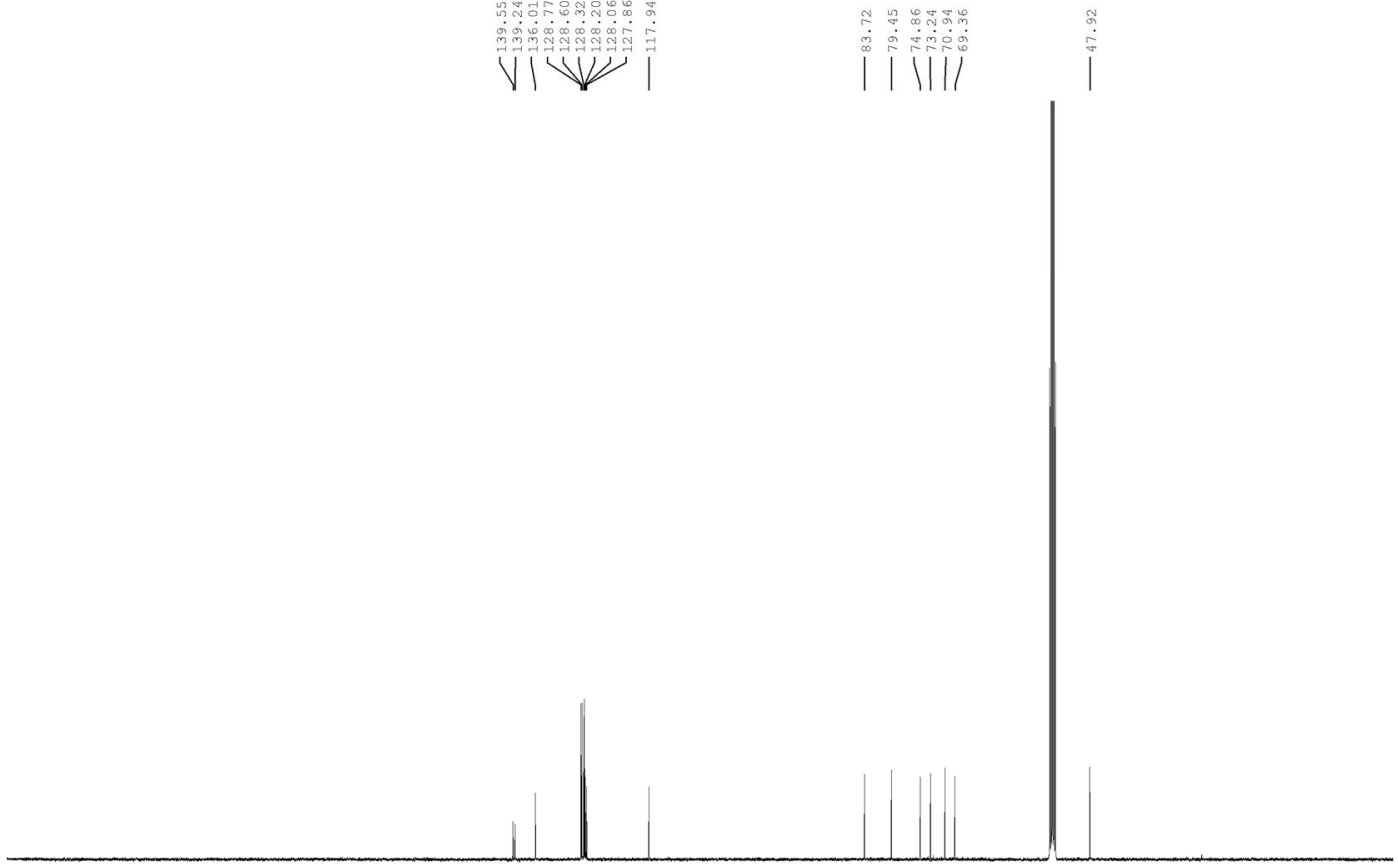

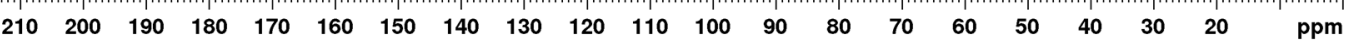


${ }^{1} \mathrm{H}$ NMR (500 MHz, $\mathrm{CD}_{2} \mathrm{Cl}_{2}$ )
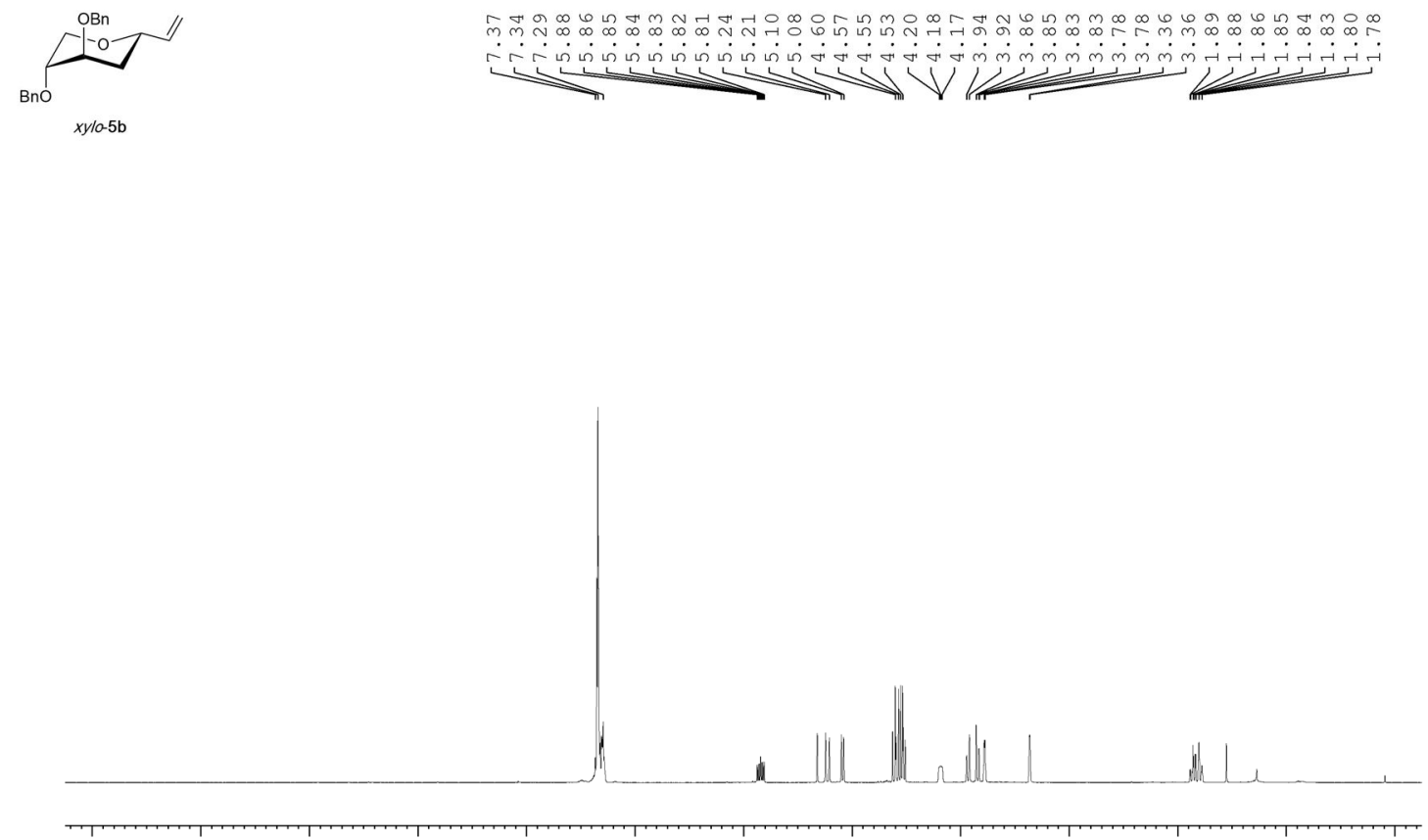

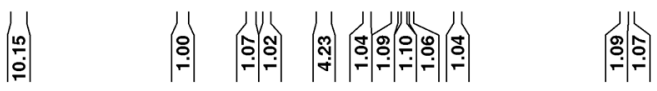

${ }^{13} \mathrm{C}$ NMR $\left(125 \mathrm{MHz}, \mathrm{CD}_{2} \mathrm{Cl}_{2}\right)$

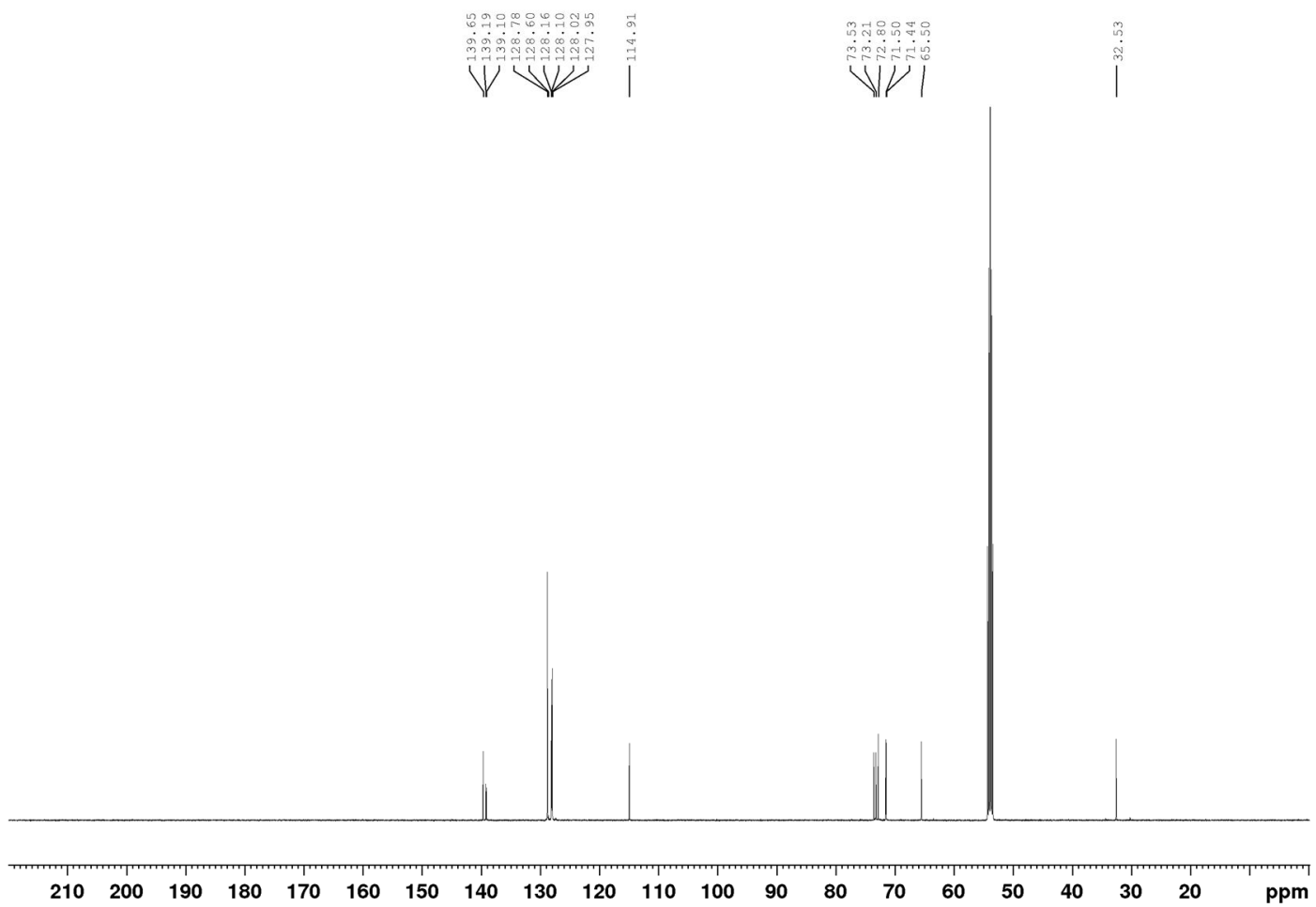

